\author{
Mon og r a p h \\ urn:1sid:zoobank.org:pub:D20CE35E-08CF-41EE-9700-ABDEA6F4413E
}

\title{
Taxonomy of the Crematogaster degeeri-species-assemblage in the Malagasy region (Hymenoptera: Formicidae)
}

\author{
Bonnie B. BLAIMER ${ }^{1} \&$ Brian L. FISHER ${ }^{2}$ \\ ${ }^{1}$ Department of Entomology, National Museum of Natural History, \\ Smithsonian Institution, Washington, DC, USA \\ E-mail: bonnieblaimer@gmail.com (corresponding author) \\ ${ }^{1,2}$ Department of Entomology, California Academy of Sciences, San Francisco, California, USA \\ ${ }^{1}$ urn:Isid:zoobank.org:author:2ADA98B1-6FEC-446E-B056-9244F417F0BC \\ ${ }^{2}$ urn:lsid:zoobank.org:author:7CFCBBE5-5443-4F94-B2AC-04BFD4D520F0
}

\begin{abstract}
We revise the species-level taxonomy of the Crematogaster (Crematogaster) degeerispecies-assemblage, a group of related ants occuring in Madagascar and the wider Malagasy region, and further provide an identification key to all species-groups of the genus Crematogaster in this region. Within the $C$. degeeri-assemblage, we recognize twelve species based upon morphological data from worker, queen and male ants, as well as genetic data from the barcode region of cytochrome oxidase I. Seven new species are described: Crematogaster alafara Blaimer sp. nov., C. bara Blaimer sp. nov., C. mafybe Blaimer sp. nov., C. maina Blaimer sp. nov., C. malahelo Blaimer sp. nov., C. masokely Blaimer sp. nov., C. ramamy Blaimer sp. nov. Crematogaster tricolor Gerstäcker, 1859 (stat. rev.) and C. dentata Dalla Torre, 1893 (stat. nov.) are raised to species level, and the following new synonymies are proposed: Crematogaster degeeri lunaris Santschi, 1928 as a synonym of C. degeeri Forel, 1886; Crematogaster sewelli improba Forel, 1907 and C. sewelli mauritiana Forel, 1907 as synonyms of $C$. dentata Dalla Torre, 1893, and C. pacifica Santschi, 1919 as a synonym of C. lobata Emery, 1895. Species descriptions, images, and distribution maps and identification keys based on worker ants, as well as on queen ants where available, are presented for all twelve species. In addition, we present a molecular gene tree for cytochrome oxidase I and summarize levels of sequence divergence within and between species of the $C$. degeeri-species-assemblage. Our findings are discussed in the light of previous work on Malagasy Crematogaster ants.
\end{abstract}

Keywords. Crematogaster, Malagasy region, ants, species-groups, barcoding.

Blaimer B. \& Fisher B.L. 2013. Taxonomy of the Crematogaster degeeri-species-assemblage in the Malagasy region (Hymenoptera: Formicidae). European Journal of Taxonomy 51: 1-64. http://dx.doi.org/10.5852/ejt.2013.51

\section{Introduction}

Crematogaster Lund, 1831 ants are a species-rich clade commonly known as "acrobat ants". This group currently includes more than 460 existing nominal species names (Blaimer 2012d; Bolton 2012) and is distributed worldwide. High intraspecific morphological variation renders the species-level taxonomy of acrobat ants challenging (Blaimer 2010, 2012a, Blaimer \& Fisher 2013). The situation 
is further complicated by many synonyms and intraspecific names (Brown 1973). On the one hand, many widespread species are clearly in need of taxonomic revision (Longino 2003; Ward 2007, 2010), while on the other hand, a large portion of the more locally endemic species remain to be discovered or described in this genus (e.g. Blaimer 2010, 2012c; Hosoishi \& Ogata 2009, 2012; Longino 2003, Blaimer \& Fisher 2013). Nomenclatural problems make identification of the Afrotropical Crematogaster fauna particularly unmanageable, and much of the diversity of these ants in this region probably has not yet been revealed.

Within the Afrotropics, Madagascar has become a flagship for progress in ant taxonomy (e.g. HitaGarcia \& Fisher 2011, 2012; LaPolla et al. 2010; Yoshimura \& Fisher 2012), since recent comprehensive inventories have created a solid basis for revisionary work (Fisher 2003, 2005, 2008). Based on the large quantity of specimens and data collected in these surveys, the Crematogaster of Madagascar have been undergoing revision in a series of taxonomic treatments (Blaimer 2010; Blaimer 2012a, 2012c, Blaimer \& Fisher 2013). Moreover, the evolution of these ants has recently been investigated via molecular phylogenetics. The findings reveal that Madagascar was colonized by Crematogaster most likely from Africa in at least eight to nine (Blaimer 2012b, 2012c) independent dispersal events. These events gave rise to six species-groups and assemblages and three phylogenetically more isolated species: the Crematogaster (Crematogaster) hova-group ( 7 species), the $C$. (Crematogaster) ranavalonae-group (3 species), the $C$. (Crematogaster) kelleri-group (6 species), the $C$. (Orthocrema) madecassa-group (2 species), the $C$. (Orthocrema) volamena-group (2 species), the $C$. (Crematogaster) degeeri speciesassemblage (12 species, including the more distantly related C. tricolor Gerstäcker, 1859 stat. rev.), C. rasoherinae Forel, 1891 and C. tsisitsilo Blaimer, 2013. Together, these comprise 34 described and yet undescribed species of Crematogaster in Madagascar.

The present study focuses on the $C$. degeeri-species-assemblage, a group that we choose here to call an "assemblage" rather than a species-group since no morphological characters that unite these ants have been discovered to date. This group was referred to in Blaimer (2012b) as the "Crematogaster degeerisewellii group"; after subsequent broadening by Blaimer (2012d) to include a few African species, it was hence dubbed the "C. castanea-group". This grouping likely does not form a clade, but rather represents a group of loosely related species that may have closer affinities with African species (but see phylogeny in Blaimer 2012b). We choose here to revert to a description of the Malagasy species in this group only, since our taxonomic insight into the related African species is not yet sufficient. Most species in the C. degeeri-assemblage occur in Madagascar only, but three species are also found in the wider Malagasy region, including the Indian Ocean Islands of Comoros, Mayotte and Mauritius. The group is not present in the Seychelles.

The natural history and ecology of species within this group do not follow a common theme but are highly diverse. The $C$. degeeri-assemblage includes arboreal species nesting in dead twigs or branches, as well as ground-dwelling species found in nests under stones or in the soil. Ground-nesting is rather atypical for Crematogaster ants, which often dominate the canopy. All major macrohabitats are occupied by one or the other representative of the $C$. degeeri-assemblage in Madagascar: dry and spiny forests, littoral forest, rainforest, montane forest, savannah and grasslands, as well as coastal and mangrove habitat. A few species further appear to adapt quite well to urban or disturbed areas.

In the following, we present a comprehensive taxonomic revision of the $C$. degeeri-species-assemblage, depending both upon morphological and on mitochondrial genetic data. In summary and addendum to prior work, we further provide an identification key to all of the aforementioned species-groups and species of Crematogaster in Madagascar, and hereby conclude the series of taxonomic work on this genus in Madagascar. 


\section{Material and methods}

\section{Morphological study}

Morphological observations were made on worker ants, as well as queen and male ants where available, with a Leica MZ9.5, MZ12.5 and MZ Apo stereomicroscope. Standard measurements (in mm) were taken at 16-50× with a Leica MZ Apo stereomicroscope and a dual-axis Acu-Rite Quikcount micrometer wired to a digital readout. Measurements are given to the second decimal place, indices are presented as decimal fractions (also to the second decimal) and ranges express minimum - maximum values. Measured specimens were chosen to represent the entire respective species distribution range and morphological variation. The abbreviations used for measurements and indices are explained below; illustrations can be found in Blaimer (2010; 2012a, 2012c).

$\mathrm{CI}=$ Cephalic index: HW/HL.

$\mathrm{EL}=$ Eye length; measured along the maximum diameter.

$\mathrm{HL}=$ Head length; perpendicular distance from line tangent to rearmost points of vertex margin to line tangent to anterior most projections of clypeus, in full face view.

$\mathrm{HW}=$ Maximum head width including eyes, in full face view.

LBI $=$ Leg-body index: WL/LHT.

LHT = Length of metatibia, excluding the proximomedial condyle.

$\mathrm{OI}=$ Ocular index: EL/HL.

PPL = Postpetiole length; measured in dorsal view at an angle that maximizes length.

PPI = Postpetiole width index: PPW/PPL.

PPW $=$ Postpetiole width; measured in same view as and perpendicular to postpetiole length.

$\mathrm{PTH}=$ Petiole height; measured in lateral profile as vertical distance from ventral margin to highest point of dorsoposterior margin.

PTHI $=$ Petiole height index: PTH/PTL.

PTL = Petiole length; measured in lateral profile as the distance from dorsoposterior margin of segment to anterior inflection point where petiole curves up to condyle.

PTW = Petiole width; maximum width of petiole in dorsal view.

PTWI $=$ Petiole width index: PTW/PTL.

SI $=$ Scape index: SL/HW.

$\mathrm{SL}=$ Scape length; length of scape shaft from apex to basal flange, not including basal condyle and neck. If scape is strongly arched, this measurement is taken as the chord length from the basal flange to the apex.

SPI $=$ Propodeal spine index: SPL/WL.

$\mathrm{SPL}=$ Propodeal spine length; measured from tip of propodeal spine to closest point on outer rim of propodeal spiracle, maximizing spine length in lateral view.

$\mathrm{WL}=$ Weber's length; measured in lateral profile of mesosoma, distance from approximate inflection point, where downward sloping pronotum curves into anteriorly projecting neck, to posteroventral propodeal lobes.

Queen and male specific measurements:

MSNI $=$ Mesonotal index: MSNW/MSNL.

MSNL = Mesonotal length; maximum length of mesonotum, measured in dorsal view.

MSNW = Mesonotal width; maximum width of mesonotum, measured in dorsal view.

Color images were created with a JVC KY-F75U digital camera, a Leica MZ16A stereomicroscope, Syncroscopy Auto-Montage (v5.0) and ZERENE STACKER (v1.04) software. All ant images presented here are also publicly available on AntWeb (www.antweb.org). All distribution maps presented here 
were plotted with the packages "maps" and "mapdata" within the software R (R Development Core Team 2008), based on collection coordinates (latitude and longitude) of all material examined. Due to the extent of material examined we decided to provide a full list of collection data for all species in the supplementary material only (see Supplementary Material 1). For material lacking primary collection information, i.e. syntype specimens and older material, the following sources were used to georeference collection sites: the GEOnet Names Server (National Geospatial-Intelligence Agency) and the Gazetteer to Malagasy Botanical Collecting Localities (Schatz \& Lescot 2012). Classification of major geographic regions in Madagascar throughout species descriptions follows Gautier \& Goodman (2003).

\section{Nomenclatural Acts}

The electronic edition of this article conforms to the requirements of the amended International Code of Zoological Nomenclature (International Commission on Zoological Nomenclature 1999), and hence the new names contained herein are available under that Code. This published work and the nomenclatural acts it contains have been registered in ZooBank, the online registration system for the ICZN. The LSID for this publication is: urn:Isid:zoobank.org:pub:D20CE35E-08CF-41EE-9700-ABDEA6F4413E. The electronic edition of this work was published in a journal with an ISSN, and has been archived and is available from the following digital repositorie: LOCKSS.

The International Commission on Zoological Nomenclature requires lectotypes designated after 1999 to "contain an express statement of deliberate designation" (amended Article 74.7.3). We use the statement "lectotype by present designation" to fulfill this requirement. Lectotypes have been designated where a name lacks a holotype or lectotype and unambiguous syntypes have been identified. The purpose is to provide stability of nomenclature, and designation is done in a revisionary context in agreement with the amended Recommendation 74G of Article 74.7.3.

\section{Specimens were examined and/or deposited in the following collections:}

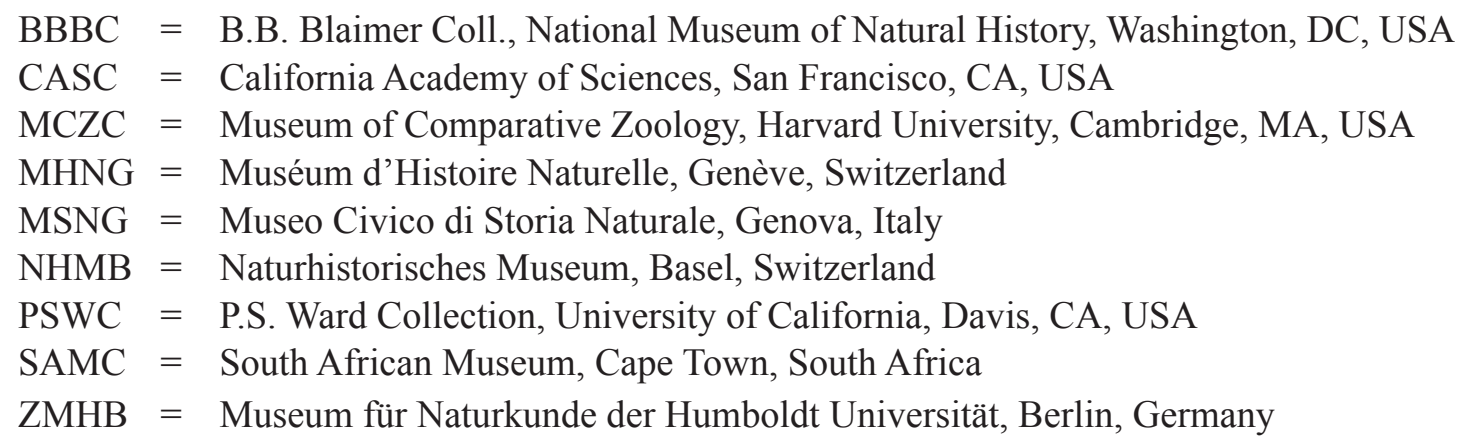

\section{Molecular data collection and analyses}

For mitochondrial genetic analysis DNA was extracted destructively from the legs of worker ants at the Biodiversity Institute of Ontario, University of Guelph under the Barcode of Life Initiative, using extraction protocols as outlined in Smith \& Fisher (2009). One to 33 specimens for the twelve species treated here were used for extractions. In general, the number of specimens per species submitted to the Barcode of Life Initiative was much larger, but success rates were poor overall due to the advanced age of specimens. Sequence data were collected for cytochrome oxidase I (COI, 592 bp used for inference) at the Biodiversity Institute of Ontario, with amplification and sequencing protocols as detailed in Smith \& Fisher (2009). Three Malagasy Crematogaster species from different species-groups were chosen as outgroups, C. madecassa Emery, 1895, C. ranavalonae Forel, 1887 and the C. hova-complex. These range from moderately to distantly related to the focal group (Blaimer 2012b). For the geographic distribution of the sampled taxa refer to Supplementary Material 2. 
BLAIMER B. \& FISHER B.L., The Crematogaster degeeri-species-assemblage

Table 1. GenBank accession numbers and voucher specimens.

\begin{tabular}{|c|c|c|c|c|c|c|c|c|}
\hline Crematogaster & voucher specimen & accession & Crematogaster & voucher specimen & accession & Crematogaster & voucher specimen & accession \\
\hline alafara & CASENT0492838 & HM418787 & maina & CASENT0107367 & HM418731 & sewellii & CASENT0206185 & KC501961 \\
\hline bara & CASENT0208888 & KC501979 & maina & CASENT0107707 & DQ176072 & sewellii & CASENT0209236 & KC501962 \\
\hline bara & CASENT0209137 & KC501980 & maina & CASENT0120361 & KC501988 & sewellii & CASENT0209261 & KC501957 \\
\hline bara & CASENT0491170 & HM418784 & maina & CASENT0138039 & HQ925455 & sewellii & CASENT0210000 & KC501958 \\
\hline degeeri & CASENT0071423 & KC501978 & maina & CASENT0146407 & GU710979 & sewellii & CASENT0496779 & HM418789 \\
\hline degeeri & CASENT0107466 & HM880704 & maina & CASENT0146414 & GU710978 & tricolor & CASENT0076476 & HM418730 \\
\hline degeeri & CASENT0120677 & HM418734 & maina & CASENT0155973 & JN283111 & tricolor & CASENT0114405 & KC501972 \\
\hline degeeri & CASENT0122383 & HM418745 & maina & CASENT0156620 & JN283133 & tricolor & CASENT0120922 & HM418736 \\
\hline degeeri & CASENT0125324 & HM418750 & maina & CASENT0156888 & JN283148 & tricolor & CASENT0120949 & HM418737 \\
\hline degeeri & CASENT0128356 & HM418752 & maina & CASENT0193387 & HQ547858 & tricolor & CASENT0121060 & HM418738 \\
\hline degeeri & CASENT0128363 & HM418745 & maina & CASENT0193388 & HQ547859 & tricolor & CASENT0122066 & HM418742 \\
\hline degeeri & CASENT0218965 & HQ925353 & maina & CASENT0193398 & HQ547860 & tricolor & CASENT0122269 & KC501973 \\
\hline dentata & CASENT0003915 & HM418715 & maina & CASENT0193399 & HQ547861 & tricolor & CASENT0122274 & HM418743 \\
\hline dentata & CASENT0059574 & EF609793 & maina & CASENT0204443 & KC501975 & tricolor & CASENT0123050 & HM418748 \\
\hline dentata & CASENT0059579 & EF609796 & maina & CASENT0204445 & KC501976 & tricolor & CASENT0132240 & KC501981 \\
\hline dentata & CASENT0059761 & EF609804 & maina & CASENT0209210 & KC501977 & tricolor & CASENT0132258 & KC501984 \\
\hline dentata & CASENT0059870 & EF609811 & maina & CASENT0209221 & KC501974 & tricolor & CASENT0132767 & KC501987 \\
\hline dentata & CASENT0059871 & EF609814 & maina & CASENT0436047 & HM418775 & tricolor & CASENT0132771 & KC501985 \\
\hline dentata & CASENT0070942 & KC501964 & maina & CASENT0484986 & HM418778 & tricolor & CASENT0134116 & HM418756 \\
\hline dentata & CASENT0071738 & KC501967 & maina & CASENT0485307 & HM418780 & tricolor & CASENT0134341 & GU709621 \\
\hline dentata & CASENT0107098 & KC501965 & maina & CASENT0490152 & HM418782 & tricolor & CASENT0134342 & GU709620 \\
\hline dentata & CASENT0120373 & HM418733 & maina & CASENT0496596 & HM418788 & tricolor & CASENT0134346 & GU709623 \\
\hline dentata & CASENT0137569 & HQ925454 & malahelo & CASENT0125705 & HM879914 & tricolor & CASENT0134362 & GU709624 \\
\hline dentata & CASENT0138306 & HQ925456 & malahelo & CASENT0141211 & HQ925457 & tricolor & CASENT0134838 & KC501982 \\
\hline dentata & CASENT0208601 & KC501970 & malahelo & CASENT0141212 & HQ925458 & tricolor & CASENT0134950 & KC501986 \\
\hline dentata & CASENT0208678 & KC501966 & masokely & CASENT0004906 & GU710477 & tricolor & CASENT0134964 & KC501983 \\
\hline dentata & CASENT0209207 & KC501968 & masokely & CASENT0068954 & HM879913 & tricolor & CASENT0136333 & HM418757 \\
\hline dentata & CASENT0209223 & KC501969 & ramamy & CASENT0021958 & HM418720 & tricolor & CASENT0136515 & GU709628 \\
\hline dentata & CASENT0484998 & HM418779 & ramamy & CASENT0049627 & HM418725 & tricolor & CASENT0136639 & GU709629 \\
\hline dentata & CASENT0490355 & HM418783 & ramamy & CASENT0137653 & HM418763 & tricolor & CASENT0136684 & GU709630 \\
\hline lobata & CASENT0040949 & HM418721 & ramamy & CASENT0137876 & HM418766 & tricolor & CASENT0137360 & GU709637 \\
\hline lobata & CASENT0041264 & HM418722 & ramamy & CASENT0147704 & GU714795 & tricolor & CASENT0137615 & HM418759 \\
\hline lobata & CASENT0073517 & HM418729 & ramamy & CASENT0156746 & JN283139 & tricolor & CASENT0137623 & HM418760 \\
\hline lobata & CASENT0156861 & JN283145 & ramamy & CASENT0157292 & JN283185 & tricolor & CASENT0137632 & GU710479 \\
\hline lobata & CASENT0193036 & HM418773 & sewellii & CASENT0121076 & HM418739 & tricolor & CASENT0137650 & HM418762 \\
\hline lobata & CASENT0484854 & HM418777 & sewellii & CASENT0121077 & HM418740 & tricolor & CASENT0137830 & HM418764 \\
\hline mafybe & CASENT0107856 & HQ925451 & sewellii & CASENT0125862 & HM418751 & tricolor & CASENT0138272 & HM418768 \\
\hline mafybe & CASENT0122362 & HM418744 & sewellii & CASENT0128361 & HM418753 & tricolor & CASENT0191124 & HM418771 \\
\hline mafybe & CASENT0193017 & HQ925460 & sewellii & CASENT0128802 & HM418755 & tricolor & CASENT0209220 & KC501971 \\
\hline mafybe & CASENT0409618 & HQ925462 & sewellii & CASENT0203837 & KC501963 & hova-complex & CASENT0193380 & HQ547857 \\
\hline maina & CASENT0003222 & HM418714 & sewellii & CASENT0204541 & KC501959 & madecassa & CASENT0068164 & KC501955 \\
\hline maina & CASENT0052467 & HM418726 & sewellii & CASENT0206148 & KC501960 & ranavalonae & CASENT0193531 & HQ547877 \\
\hline
\end{tabular}


Table 2. Data partitions and selected substitution models. Data subsets used in phylogenetic analyses and their characteristics. $\mathrm{VC}=$ variable characters; PIC = parsimony informative characters; [ ] = ingroup only. The partitioning scheme and the respective substitution models were chosen under the AIC with the software PartitionFinder (Lanfear et al. 2012).

\begin{tabular}{|c|c|c|c|c|}
\hline Data partition & No. bases & No. VC & No. PIC & Substitution model \\
\hline COI positions 1+2 & 395 & $14[3]$ & $44[38]$ & HKY+I+G \\
\hline COI positions 3 & 197 & $17[10]$ & $171[163]$ & GTR+G \\
\hline Total & $\mathbf{5 9 2}$ & $\mathbf{3 1}[\mathbf{1 3}]$ & $\mathbf{2 1 5}[\mathbf{2 0 1}]$ & \multicolumn{1}{|c}{} \\
\cline { 1 - 3 } & &
\end{tabular}

All newly generated sequences have been deposited in GenBank, and accession numbers are listed in Table 1; the data matrix and trees used to create Figs 1 and 2 have further been deposited in TreeBase under the accession http://purl.org/phylo/treebase/phylows/study/TB2:S14163. Sequence data were aligned with MUSCLE (Edgar 2004), accessed through the CIPRES science gateway (Miller et al. 2010). The dataset was subdivided into three data subsets according to codon positions. We then used PartitionFinder (Lanfear et al. 2012) to simultaneously choose the best-scoring partition scheme and to select best-fitting models of nucleotide sequence evolution for each data subset (under the AIC, Akaike Information Criterion). The selected models for the chosen partition scheme can be found in Table 2 .

Phylogenetic analyses were performed within a Bayesian framework using MRBAYES v3.2 (Ronquist et al. 2012), accessed through the CIPRES science gateway (Miller et al. 2010), and within a maximum likelihood framework using GARLI v2.0 (Zwick1 2006), accessed through the GARLI Web Service (http://molecularevolution.org). Bayesian analyses each employed two runs of Metropolis-coupled Markov Chain Monte Carlo (MCMCMC) consisting of four chains (temp $=0.05$ ) and sampling every 1000 generations. The model parameters transition-transversion ratio, gamma shape, proportion of invariable sites, rate matrix and state frequencies were unlinked across partitions. A dirichlet rateprior $(1,5)$ was used and the branch length prior settings were modified (unconstrained:exponential (100)) to obtain realistic values for rate multipliers and tree length. In MrBayes output files, we confirmed that the ASDSF (Average Standard Deviation of Split Frequencies) had reached values well below 0.01 and PSRF (Potential Scale Reduction Factor) values had approached 1.0 for all parameters. In TRACER v1.5 (Rambaut \& Drummond 2007), convergence was confirmed visually and mixing of chains was evaluated with effective sample size (ESS) values. To assess whether tree topologies were sampled in proportion to their true posterior distribution, we further used the compare, slide and cumulative plotting functions on the AWTY-online server (Nylander et al. 2008). All the above indicators returned good values after MCMCMC-sampling for 10 million generations; consensus trees were summarized in MrBayes after discarding $25 \%$ of samples as burnin. A maximum likelihood bootstrap search with 100 replicates was performed in GARLI, with the resulting trees summarized as a majority-rule consensus tree. All trees were rooted with the outgroup method using a more distantly related taxon, Crematogaster madecassa (see Blaimer 2012b). We used FigTree 1.3.1 (Rambaut 2006-2009) for tree visualization and then exported these into AdobeIllustrator CS5 to create our figures as displayed here.

Mean, minimum and maximum sequence divergences of COI within and between species were calculated under the Tamura-Nei model (Tamura \& Nei 1993) with the software MEGA5 (Tamura et al. 2011). 


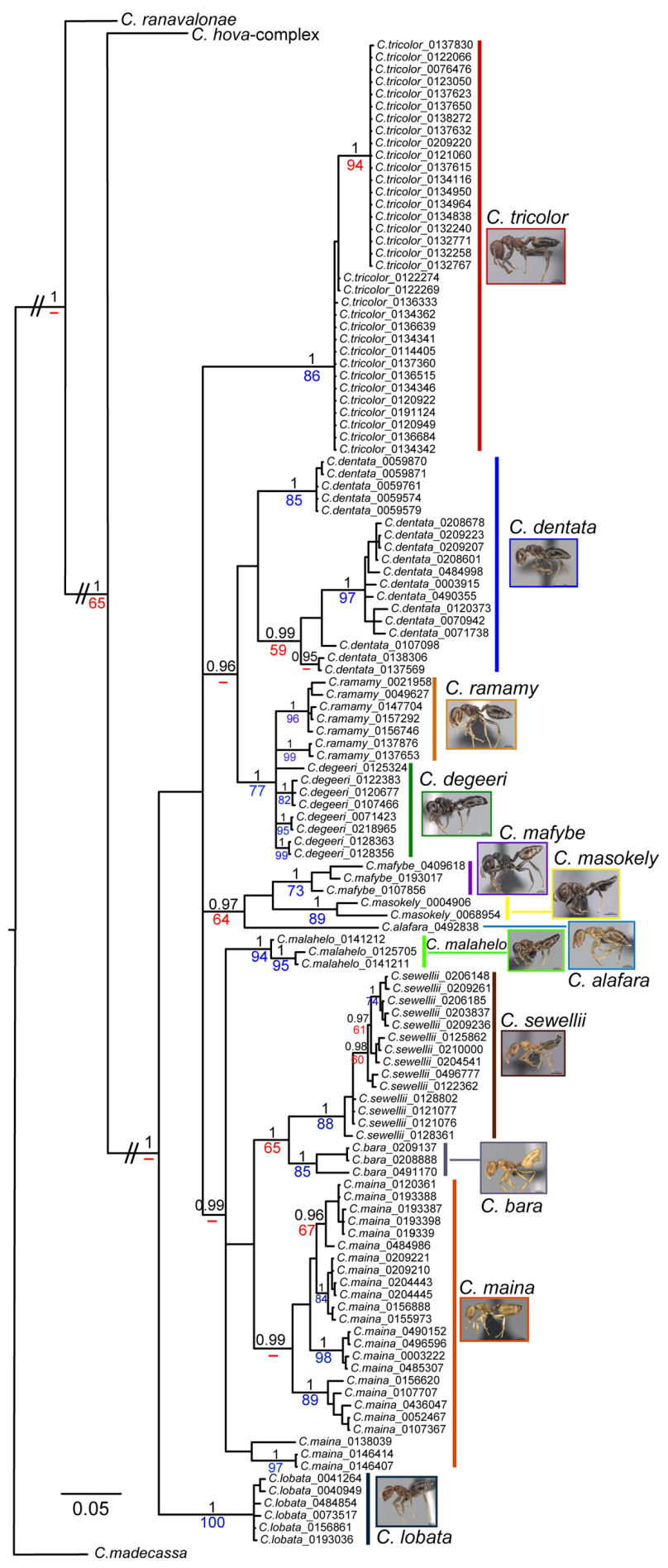

Fig. 1. Mitochondrial phylogeny of species in the Crematogaster degeeri-assemblage. Results of Bayesian inference based on 592 bp of COI, summarized as a consensus tree in MrBayes. Support values in black represent posterior probabilities (pp), those in blue ML (Maximum Likelihood) bootstrap values $>70$, and those in red ML bootstrap values $<70$. Values $p p<0.96$ are not shown; nodes not present in the ML analysis are represented by a dash instead of a bootstrap value. The scale bar shows nucleotide changes per base pair. Long branches subtending the outgroup taxa C. madecassa Emery, 1895, C. ranavalonae Forel, 1887 and $C$. hova-complex have been shortened. Appended numbers on taxon labels represent CASENT\#'s of respective voucher specimens. 


\section{Results}

\section{Molecular taxonomy}

In this study, we used $592 \mathrm{bp}$ of the barcoding region of cytochrome oxidase I to corroborate our hypotheses on species boundaries in the $C$. degeeri-species-assemblage formed from morphological observations. We present the results of this molecular analysis 1) in the form of a phylogenetic tree (Fig. 1), and 2) as a distance matrix of sequence divergences within and between species (Table 3). We further illustrate the relationships of populations between Madagascar and the wider Malagasy region where applicable (Fig. 2). The phylogenies shown here are results from Bayesian inference, with bootstrap values from maximum likelihood analyses mapped on the relevant nodes. These trees represent a single mitochondrial gene tree and should not be mistaken as species-level phylogenies depicting relationships between these ants.

Except for four cases (C. dentata Dalla Torre, 1893 stat. nov., C. maina sp. nov., C. degeeri Forel, 1886, C. ramamy sp. nov.), we found good evidence for our putative species, and phylogenetic support values ranged from posterior probabilities (pp) $=1$ and bootstraps between 73-100 (Fig. 1). Excluding the unsupported species, mean within-species sequence divergence was $<6.1 \%$, and mean between-species sequence divergence ranged between $7.9-22.2 \%$.

Our analysis recovered Crematogaster degeeri and C. ramamy sp. nov. as not reciprocally monophyletic, but forming a grade (Fig. 1). Mean sequence divergence between the two species was $4.7 \%$ (Table 3 ), and thus lower than the maximum within-species divergence in C. ramamy sp. nov. (6.6\%). However, our morphological and ecological observations here outweigh these results and we did not alter our decision to separate these into two distinct species.

In the case of Crematogaster maina sp. nov. we found weak support that sequenced individuals of this species from Madagascar form a genetic clade, which did, however, not cluster with the Comoros population (Fig. 1 and Fig. 2A). The two individuals sequenced from the Comoros cluster (albeit without much support) along with an individual from a small island off the northern Madagascar coast, Nosy Faly (Fig. 2A), show 9.8-13.3\% sequence divergence with the Madagascar mainland population (Table 3 ; only maximum value shown). We could not find correlated morphological differences between these two genetic clusters, and were reluctant to distinguish separate species on the basis of only two to three mitochondrial sequences that could potentially be compromised in quality (see discussion). Our new species Crematogaster maina sp. nov. as described below thus includes both the Madagascar and Comoros populations.

A similar case is seen in $C$. dentata stat. nov. Virtually no support is recovered for a cluster of sequences that includes both individuals from Madagascar and Mauritius (Fig. 2B), and the sequence divergence between the two clusters was found to be $8.8-11.2 \%$. We again choose to include the Mauritius population in the species definition of $C$. dentata stat. nov. and discuss this decision below in the context of species descriptions and the general discussion.

However, a very different picture is seen in Crematogaster tricolor stat. rev., the other species in the $C$. degeeri-assemblage distributed beyond Madagascar in the wider Malagasy region (Fig. 2C). Here we find an astonishingly level of low genetic divergence within the species as a whole (Table 3 ; mean $1.8 \%$, max. $3.9 \%$ ). Furthermore, an interesting genetic structure emerges from the sequence data: one cluster contains individuals from northern and western Madagascar and all individuals sampled from Mayotte (Fig. 2C). This cluster diverges from a grade of sequences containing all individuals sampled from Comoros and from eastern Madagascar. These results suggest interesting phylogeographic relationships in C. tricolor stat. rev., and may be evidence of multiple recent dispersal events between Madagascar and the Indian Ocean Islands. 
Table 3. Summary of Tamura-Nei distance matrix. Divergences of cytochrome oxidase I sequences, as estimated under the Tamura-Nei Model (Tamura \& Nei 1993) within the software MEGA5 (Tamura et al. 2011). Bold font emphasizes highest levels of divergence within and between species.

\begin{tabular}{|c|c|c|c|c|c|c|c|c|c|c|c|c|c|c|c|}
\hline & \multicolumn{3}{|c|}{ Within species } & \multicolumn{12}{|c|}{ Between species (mean) } \\
\hline & mean & min. & $\max$. & $\begin{array}{l}0 \\
\frac{0}{3} \\
\frac{0}{3} \\
0\end{array}$ & 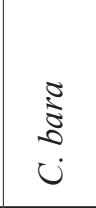 & $\begin{array}{l}-\pi \\
0 \\
0 \\
8 \\
0 \\
0 \\
ن\end{array}$ & 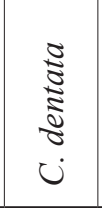 & $\begin{array}{c}\frac{\Xi}{3} \\
0 \\
0 \\
0 \\
0\end{array}$ & 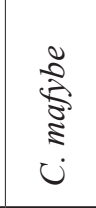 & $\begin{array}{c}\Xi \\
\stackrel{\Xi}{\Xi} \\
\vdots \\
ن\end{array}$ & 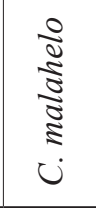 & 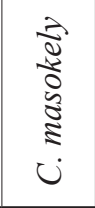 & 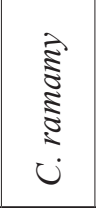 & $\begin{array}{l}: \widetilde{\Xi} \\
\frac{\Xi}{0} \\
\vdots \\
\infty \\
ن\end{array}$ & 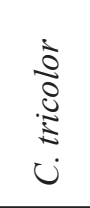 \\
\hline C. alafara & $\mathrm{n} / \mathrm{a}$ & $\mathrm{n} / \mathrm{a}$ & $\mathrm{n} / \mathrm{a}$ & 1 & & & & & & & & & & & \\
\hline C. bara & 0.028 & 0.000 & 0.042 & 0.184 & 1 & & & & & & & & & & \\
\hline C. degeeri & 0.030 & 0.000 & 0.048 & 0.177 & 0.132 & 1 & & & & & & & & & \\
\hline C. dentata & 0.066 & 0.000 & 0.112 & 0.200 & 0.160 & 0.135 & 1 & & & & & & & & \\
\hline C. lobata & 0.009 & 0.002 & 0.015 & 0.193 & 0.143 & 0.155 & 0.191 & 1 & & & & & & & \\
\hline C. mafybe & 0.043 & 0.031 & 0.061 & 0.157 & 0.139 & 0.165 & 0.160 & 0.185 & 1 & & & & & & \\
\hline C. maina & 0.062 & 0.000 & 0.133 & 0.184 & 0.113 & 0.165 & 0.176 & 0.172 & 0.169 & 1 & & & & & \\
\hline C. malahelo & 0.019 & 0.007 & 0.028 & 0.196 & 0.109 & 0.146 & 0.158 & 0.157 & 0.148 & 0.115 & 1 & & & & \\
\hline C. masokely & 0.059 & $\mathrm{n} / \mathrm{a}$ & $\mathrm{n} / \mathrm{a}$ & 0.165 & 0.139 & 0.167 & 0.189 & 0.183 & 0.128 & 0.178 & 0.163 & 1 & & & \\
\hline C. ramamy & 0.033 & 0.002 & 0.066 & 0.178 & 0.140 & 0.047 & 0.127 & 0.162 & 0.163 & 0.178 & 0.156 & 0.165 & 1 & & \\
\hline C. sewellii & 0.018 & 0.000 & 0.037 & 0.222 & 0.079 & 0.140 & 0.147 & 0.172 & 0.156 & 0.130 & 0.129 & 0.175 & 0.159 & 1 & \\
\hline C. tricolor & 0.018 & 0.000 & 0.039 & 0.215 & 0.180 & 0.166 & 0.213 & 0.199 & 0.202 & 0.222 & 0.184 & 0.218 & 0.187 & 0.206 & 1 \\
\hline \multicolumn{4}{|c|}{ outgroup: C. hova-complex } & 0.324 & 0.279 & 0.289 & 0.275 & 0.267 & 0.285 & 0.288 & 0.275 & 0.266 & 0.289 & 0.281 & 0.326 \\
\hline
\end{tabular}

\section{Checklist of species treated in this revision}

Based on both molecular and morphological taxonomic observations we therefore recognize twelve species in the $C$. degeeri-assemblage, including seven new species. Proposed synonymies are based on comparison of type specimens with morphological data.

Crematogaster alafara Blaimer sp. nov.

Crematogaster bara Blaimer sp. nov.

Crematogaster degeeri Forel, 1886

= Crematogaster degeeri var. lunaris Santschi, 1928 syn. nov.

Crematogaster dentata Dalla Torre, 1893 stat. nov.

= Crematogaster sewelli var. improba Forel, 1907 syn. nov.

= Crematogaster sewelli subsp. mauritiana Forel, 1907 syn. nov.

Crematogaster lobata Emery, 1895

= Crematogaster pacifica Santschi, 1919 syn. nov.

Crematogaster mafybe Blaimer sp. nov.

Crematogaster maina Blaimer sp. nov.

Crematogaster malahelo Blaimer sp. nov.

Crematogaster masokely Blaimer sp. nov.

Crematogaster ramamy Blaimer sp. nov.

Crematogaster sewellii Forel, 1891

Crematogaster tricolor Gerstäcker, 1859 stat. rev. 

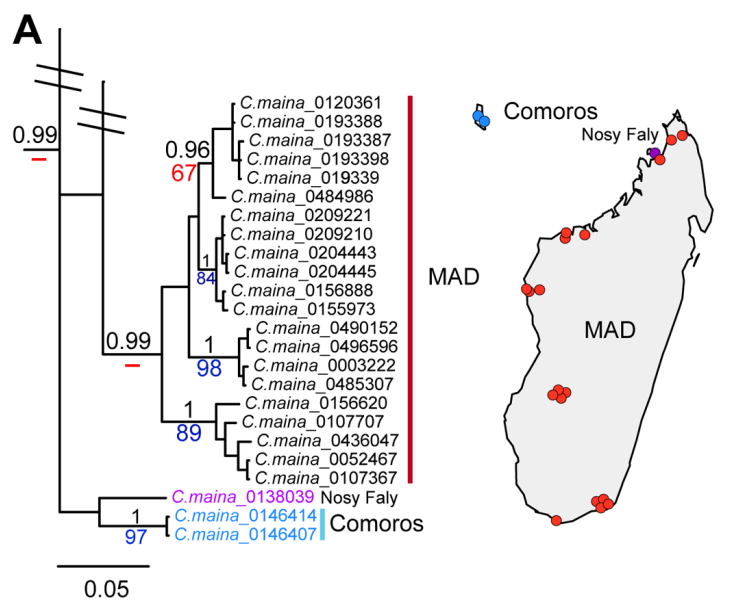

\section{C. maina}

B
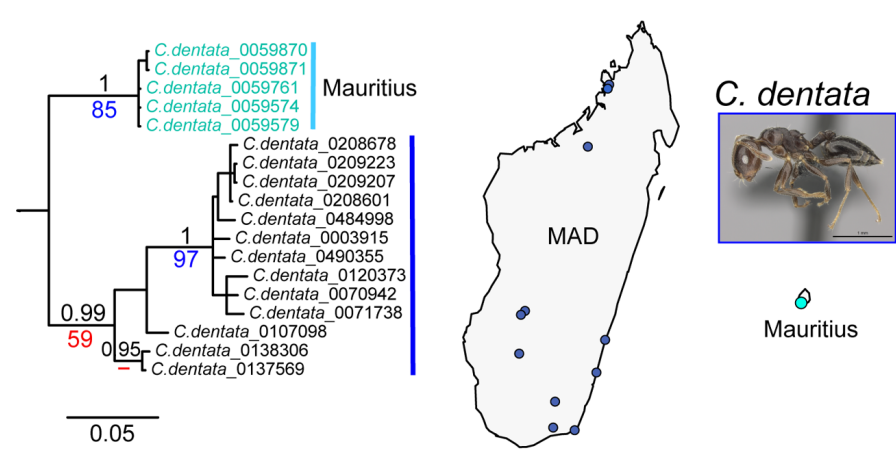

C

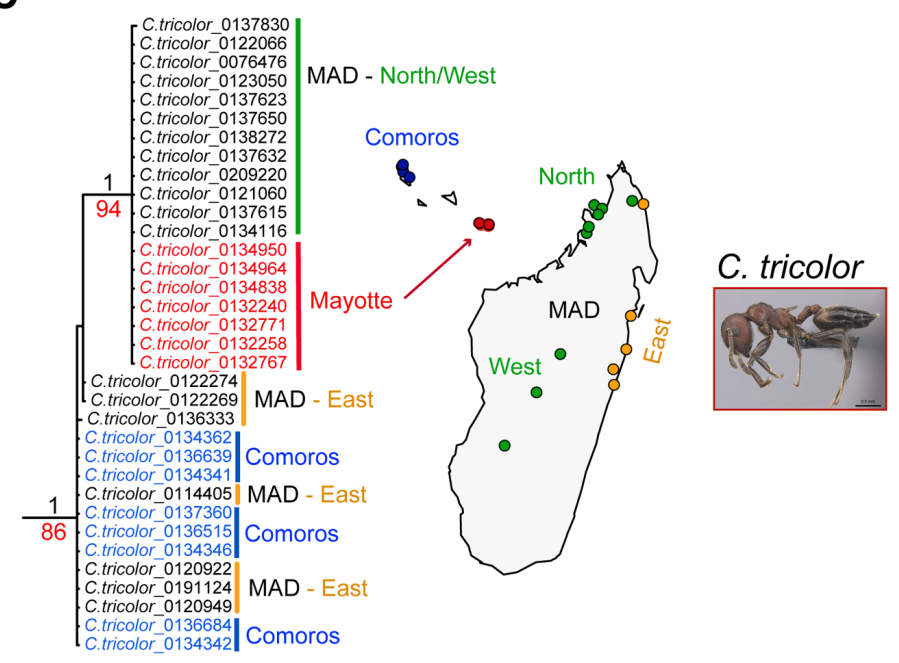

0.05

Fig. 2. Relatedness of populations between Madagascar and the wider Malagasy region. Subsets of the same phylogeny as shown in Fig. 1, with broad geographical origin of sequenced individuals mapped onto the tree for: A. Crematogaster maina sp. nov. B. Crematogaster dentata stat. nov. C. Crematogaster tricolor stat. rev. Support values in black represent posterior probabilities (pp), those in blue ML bootstrap values $>70$ and those in red ML bootstrap values $<70$. Values $p p<0.96$ are not shown; nodes not present in the ML analysis are represented by a dash instead of a bootstrap value. The scale bar shows nucleotide changes per base pair. Distance between Madagascar and Islands is not drawn to scale. Appended numbers on taxon labels represent CASENT\#'s of respective voucher specimens. 


\section{Key to subgenera and species-groups of Crematogaster in the Malagasy region}

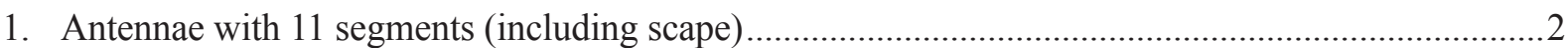

- Antennae with 10 segments (including scape). ......... Crematogaster hova-group (former subgenus Decacrema Forel, 1910; refer to Blaimer 2010)

2. Petiole ovo-rectangular (Fig. 3A), or rectangular (Fig. 3B), with postero-lateral denticles bearing long erect setae; antennae with a 2-segmented club (Fig. 3E)

subgenus Orthocrema Santschi, 1918 (refer to Blaimer 2012b)

- Petiole of different shape, but never rectangular or ovo-rectangular (as e.g. in Fig. 3C, D); antennae with a 3-segmented club (as e.g. in Fig. 18A), or antennal club indistinct (as in Fig. 3F).

3. Median portion of clypeus with a flange (cf) projecting somewhat over mandibles (Fig. 3G); promesonotal suture (pms) present and mesonotum (m) in dorsal view longer than pronotum (p) (Fig. 3H); head round (Fig. 3F); eyes situated distinctly above midline of head in full-face view (Fig. 3F).

... Crematogaster ranavalonae-group (former subgenus Oxygyne Forel, 1901; refer to Blaimer 2012a)

- Median portion of clypeus not protruding as a flange over mandibles (Fig. 4A); promesonotal suture often absent (e.g. as in Figs 7C; 12D; 15C; 18D), if present then mesonotum in dorsal view shorter than pronotum; head shape variable, rounded or quadrate (as e.g. in Figs 6A; 7A; 10A, D; 12A; $13 \mathrm{~A})$; position of eyes variable, situated at or above midline of head in full-face view.

4. Propodeal spines entirely absent (SPI 0.00), at most obtuse tubercules present (as e.g. in Figs 4B; 12C; 13B)

- Propodeal spines present (SPI >0.01), from minute dents to distinct spines (as e.g. in Figs 6B; 7B; $8 \mathrm{~B} ; 10 \mathrm{E} ; 15 \mathrm{~B} ; 17 \mathrm{C} ; 18 \mathrm{C} ; 19 \mathrm{C} ; 20 \mathrm{~B} ; 22 \mathrm{~B})$.

5. Mesonotum (m) greatly raised with respect to pronotum and propodeum (Fig. 4B); eyes larger (OI $>0.21$ ); medium to small size (HW 0.66-1.03, WL 0.62-0.98); color orange or brown.....

C. tsisitsilo Blaimer, 2013 (refer to Blaimer \& Fisher 2013)

- Promesonotum not much raised with respect to propodeum (as e.g. in Figs 12C, 13B); eyes small $(\mathrm{OI}<0.21)$ (as e.g. Figs 12A, 13A); medium to very large size (HW 0.80-1.43, WL 0.86-1.48); color brown or black

C. degeeri-species-assemblage (refer to species key in this study)

6. In lateral view promesonotum forming a straight (or at most slightly rounded) plane (Fig. 4D); posterior face of mesonotum (pfm) long, distinctly set off from dorsal face (Fig. 4C), abruptly and steeply sloping into metanotal groove (Fig. 4D); propodeal spines always spiniform, very short to medium-sized (SPI 0.070.24), situated high on propodeum (pd) (Fig. 4D).......... C. kelleri-group (refer to Blaimer \& Fisher 2013)

- Promesonotum variable, but never fitting above description; propodeal spines variable, minute denticles to distinct spines (SPI 0.01-0.26), usually situated lower on propodeum (as in Figs 6B; 7B; 8B; 10B, E; 15B; 17C; 18C; 19C) C. degeeri-species-assemblage (refer to species key in this study)

\section{Key to the workers of the $C$. degeeri-species-assemblage in the Malagasy region}

1. Propodeal spines absent, reduced to small tubercules or denticles (as in Figs 10E; 12C; 13B; 18C; 20B), or short triangular spines (as in Fig. 17C) with SPI $<0.07$; if very rarely propodeal spines spiniform and longer (SPI 0.08-0.10) (as in Fig. 6B), then small species (HW 0.72-0.88, WL 0.790.91), color dirty-yellow, montane rainforest habitats................................................................ 2

- Propodeal spines longer, distinctly spiniform (as in Figs 7B; 8B; 10B; 15B; 22B) and SPI > 0.07; size and color variable; all habitats.

2. Eyes small $(\mathrm{OI}<0.21)$ and largely confluent with lateral head margin (as in Fig. 12A; 13A; 18A) .... 3

- Eyes larger $(\mathrm{OI}>0.20)$ and distinctly protruding from lateral head margin (as in Figs $6 \mathrm{~A} ; 17 \mathrm{~A} ; 20 \mathrm{~A}) . . . . .6$ 
3. Pronotum dorsally with a distinct median longitudinal groove (Fig. 4E), medium to large species (HW 0.98-1.43, WL 0.98-1.48).....

C. mafybe sp. nov.

- Pronotum dorsally without median longitudinal groove (as e.g. Figs 4F; 18D), size variable ........ 4

4. Propodeum with spines absent or at most small tubercules (SPI 0.00) C. lobata Emery, 1895

- Propodeum with short triangular spines or sharp minute denticles

5. Antennal scapes very short (SI 0.67-0.75), not reaching head margin (as in Fig. 18A); mesonotum usually with posterolateral denticles (as in Fig. 18C).

C. masokely sp. nov.

- Antennal scapes variable, but usually longer (SI 0.69-0.82) and about reaching head margin (as in Fig. 10A, D); mesonotum usually without posterolateral denticles (as in Fig. 10B, E)

C. dentata Dalla Torre, 1893 stat. nov. [part]

6. Eyes very large (OI 0.24-0.29), situated above midline of head in full-face view (as in Fig. 20A); antennal scapes mostly longer (SI 0.77-0.87), reaching or surpassing head margin; bicolored: head and thorax yellowish to dark orange, metasoma with A4-7 brown or black (as in Fig. 20B, C) C. sewellii Forel, 1891

- Eyes mostly smaller (OI 0.20-0.26), usually situated at midline of head in full-face view (as e.g. Figs 6A; 10A, D; 17A); antennal scapes variable, but often shorter (SI 0.69-0.83), just about or not reaching head margin (as e.g. Figs $6 \mathrm{~A} ; 10 \mathrm{~A}, \mathrm{D} ; 17 \mathrm{~A}$ ); color variable

7. Propodeum usually distinctly set off from promesonotum in lateral view (Figs $6 \mathrm{~B} ; 17 \mathrm{C}$ ) and often with posterolateral denticles; promesonotum with sparse erect pilosity $(<8$ setae $)$; petiole often much wider than long (PTWI 1.18-1.52), and strongly flared (as in e.g. Fig. 3C).

- Propodeum not distinctly set off from promesonotum (in lateral view) (Fig. 10B, E), never with distinct posterolateral denticles; promesonotum usually with abundant erect pilosity ( $>8$ setae); petiole not much wider than long (PTWI 0.95-1.31), moderately flared (Fig. 3D)..

C. dentata Dalla Torre, 1893 stat. nov. [part]

8. Medium-sized species (HW 0.86-1.03, WL 0.92-1.08); propodeal spines short and triangular (SPI 0.03-0.07); pronotum with sharp lateral margins (Fig. 5A).

C. malahelo sp. nov.

- Small species (HW 0.72-0.88, WL 0.79-0.91); propodeal spines longer (SPI 0.06-0.10); pronotum lacking sharp lateral margins (Fig. 5B)....

C. alafara sp. nov.

9. Body color dirty to bright yellow (as in Fig. 7B, 15B)....

- Predominant color either orange (as in Fig. 20B), brown (e.g. as in Fig. 19C) or black (e.g. as in Fig. 8B) .11

10. Medium to large species (HW 0.87-1.03, WL 0.98-1.22); antennal scapes very long (SI 0.91-1.01), easily surpassing head margin (as in Fig. 7A).

C. bara sp. nov.

- Small species (HW 0.60-0.86, WL 0.66-0.93); antennal scapes short (SI 0.71-0.83), not or about reaching head margin (as in Fig. 15A)

C. maina sp. nov.

11. Head distinctly broader than long (CI 1.10-1.19) (as in Fig. 22A); propodeal spines longer (SPI 0.100.26); tricolored: orange-red, yellow and black (as in Fig. 22C)C. tricolor Gerstäcker, 1859 stat. rev.

- Head not much broader than long (CI 1.01-1.13); propodeal spines shorter ( $\mathrm{SPI}<0.16$ ); (as e.g. Figs 8A; 10A, D; 19A); uniformly colored or bicolored.

12. Mesonotum often with posterolateral denticles (Fig. 5C); in lateral view distinctly set off from propodeum (Fig. 5C); eyes large (OI 0.22-0.26); propodeal spines longer (SPI 0.10-0.16); erect pilosity on promesonotum often sparse ( $<8$ long erect setae) (as e.g. Figs $5 \mathrm{C} ; 8 \mathrm{~B}$ ) ......

C. degeeri Forel, 1886 
- Mesonotum variable, but more often without posterolateral denticles, and thus in lateral view not as distinctly set off from promesonotum (Fig. 5D); erect pilosity usually more abundant ( $>8$ long erect setae) (as in Fig. 5D, E); propodeal spines shorter $(\mathrm{SPI}<0.12)$.

13. Propodeum with a distinct short dorsal face, and with a transverse impression (Fig. 5E); sculpture on pronotum carinulate or reticulate (Fig. 5E); petiole oval, fairly slender; (PTWI 0.95-1.14); propodeal spines longer (SPI 0.07-0.12)

C. ramamy sp. nov.

- Propodeum usually without a distinct dorsal face, never with transverse impression (Fig. 5F); sculpture on propodeum rugulose or aciculate (Fig. 5F); petiole variable, but often wider (PTWI 0.95-1.31); propodeal spines usually shorter (SPI 0.01-0.10).

C. dentata Dalla Torre, 1893 stat. nov. [part]

\section{Key to the queens of the $C$. degeeri-species-assemblage in the Malagasy region}

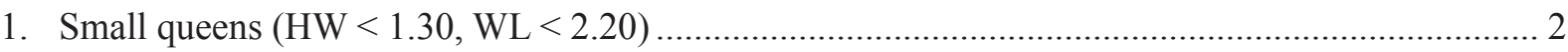

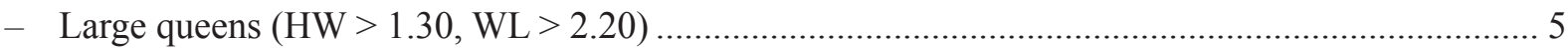

2. Head shape variable, but usually elongate, appearing longer than wide (CI 0.94-1.03) (as in Figs 6F; $11 \mathrm{~A} ; 16 \mathrm{~A})$; eyes smaller $(\mathrm{OI}<0.32)$, situated below midline of head in full-face view (as in Figs 6F; $11 \mathrm{~A} ; 16 \mathrm{~A})$; antennal scapes shorter, often not surpassing level of lateral ocelli $(\mathrm{SI}<0.67) \ldots \ldots \ldots . . . . .3$

- Head shape square, appearing as wide as long (CI 1.06); eyes larger (OI >0.33), situated at midline of head in full-face view (as in Fig. 20D); antennal scapes longer, surpassing level of lateral ocelli $(\mathrm{SI}>0.68)$ (as in Fig. 20D); C. sewellii Forel, 1891

3. Body color brown

- Body color yellow.

C. maina sp. nov.

4. Very small species (HW 1.01, WL 1.68); head sculpture finely reticulate; ocelli small and protruding very little from head surface (as in Fig. 6F)

C. alafara sp. nov.

- Larger species (HW 1.13-1.41, WL 1.73-2.43); head sculpture aciculate-shiny; ocelli distinctly protruding from head surface (as in Fig. 11A) ............ C. dentata Dalla Torre, 1893 stat. nov. [part]

5. Antennal scapes long, surpassing lateral ocelli easily and almost reaching head margin (SI $>0.76$ ) (as in Fig. 7F); body color yellow

C. bara sp. nov.

- Antennal scapes shorter (SI < 0.74) (as in Figs 8D; 13D; 22D); body color other than yellow ....... 6

6. Eyes small (OI 0.23-0.26); antennal scapes very short (SI 0.57-0.59) (as in Fig. 13D); large queens (HW 1.70-2.18, WL 2.56-3.44); color black

C. mafybe sp. nov.

- Eyes usually larger (OI 0.25-0.33) (as in Figs 8D; 22D); antennal scapes mostly longer (SI 0.570.74 ) (as in Figs 8D, 22D); usually smaller queens (HW 1.30-1.77, WL 2.20-2.69); color variable, usually not black.

7. Head distinctly wider than long (CI 1.14-1.21) (as in Fig. 22D); tricolored: reddish-orange, yellow and black (as worker in Fig. 22C); or at least abdominal segment 4 dorsally with a yellowish patch (as in Fig. 22F).

C. tricolor Gerstäcker, 1859 stat. rev.

- Head shape variable, but usually not much wider than long $(\mathrm{CI}<1.10)$ (as in Fig. 8D); uniformly colored .....

8. Eyes situated at about midline of head in full-face view (as in Fig. 8D); antennal scapes longer (SI 0.68-0.72), surpassing level of lateral ocelli.

C. degeeri Forel, 1886

- Eyes situated below midline of head in full-face view (as in Fig. 11A); antennal scapes shorter (SI 0.57-0.67), not surpassing level of lateral ocelli.....

C. dentata Dalla Torre, 1893 [part] 

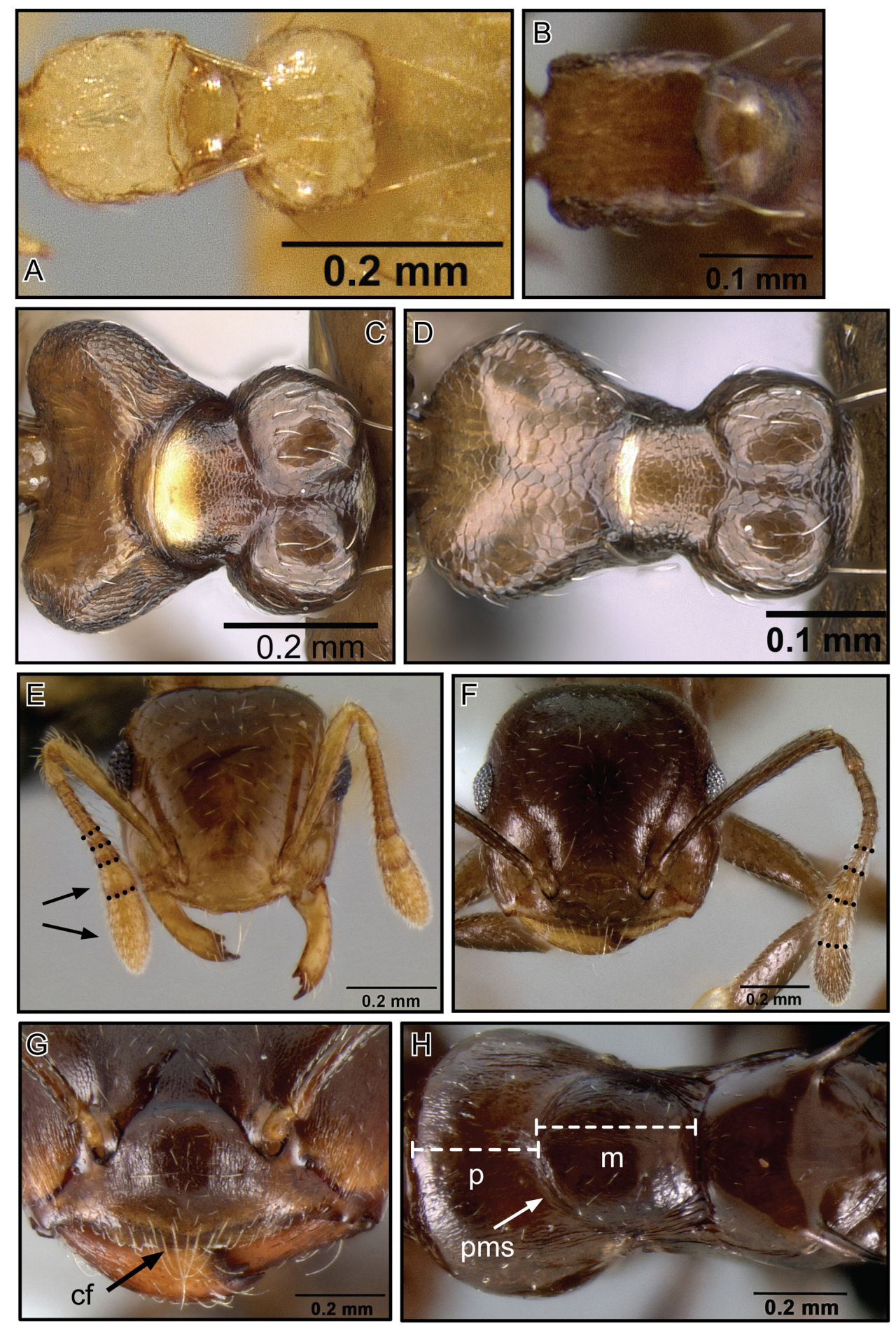

Fig. 3. Key to subgenera \& species-groups of Malagasy Crematogaster (part I). A. Petiole ovo-rectangular [C. telolafy Blaimer, 2012, CASENT0419808]. B. Petiole rectangular [C. rasoherinae Forel, 1891, CASENT0070841]. C. Petiole broadly flared [C. malahelo sp. nov., CASENT0317768]. D. Petiole oval [C. dentata sp. nov., CASENT0138289]. E. Antennal club two-segmented [C. razana Blaimer, 2012, CASENT0149655]. F. Antenna lacking distinct club [C. ranavalonae Forel, 1887, CASENT0423149]; G. Median portion of clypeus with flange (cf) [C. ranavalonae, CASENT0433703]. H. Promesonotal suture (pms) present, mesonotum (m) in dorsal view longer than pronotum (p) [C. agnetis Forel, 1892, CASENT0107473]. 

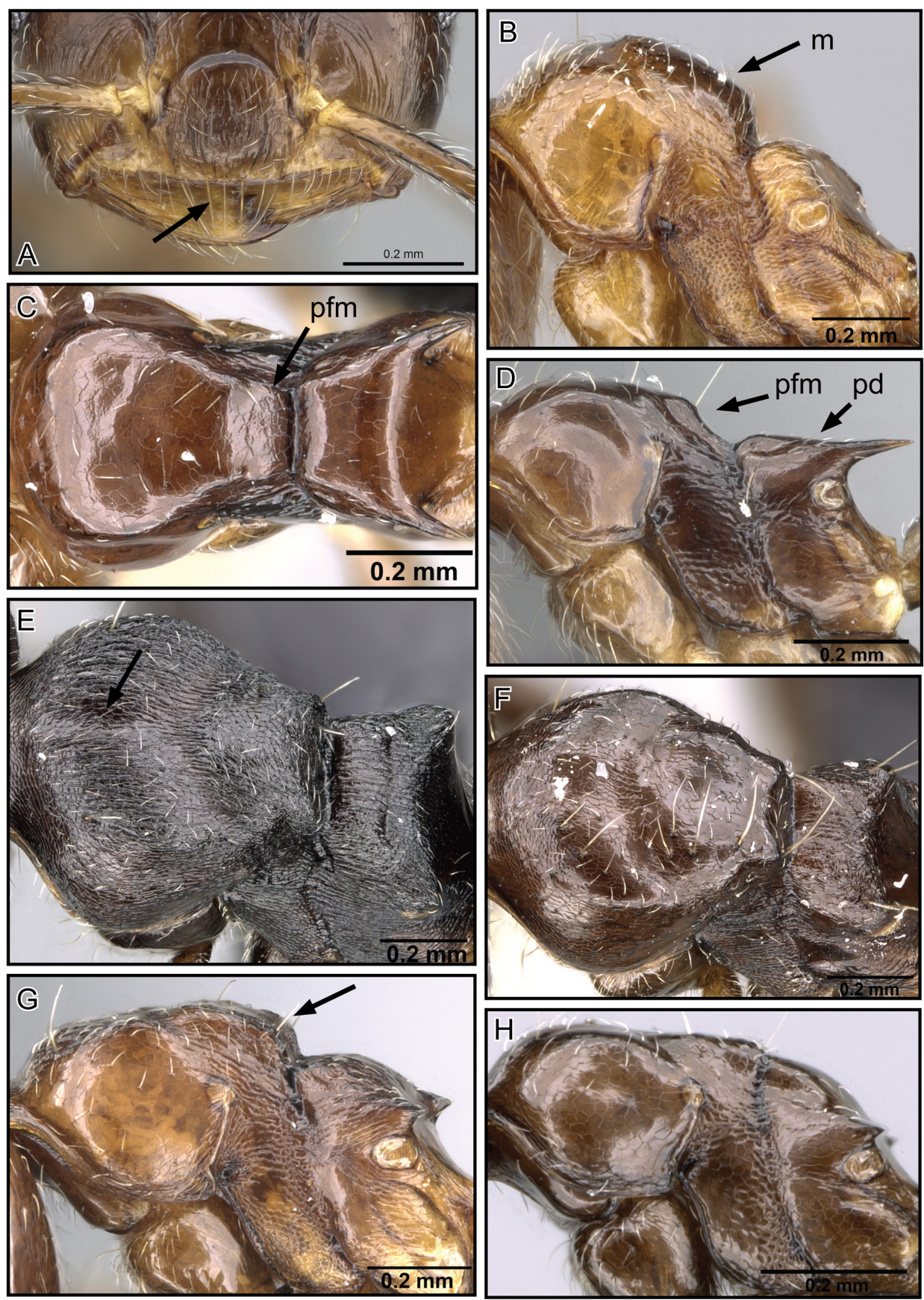

Fig. 4. Key to subgenera \& species-groups of Malagasy Crematogaster (part II); key to the species of the $C$. degeeri-assemblage (part I). A. Median portion of clypeus lacking flange [C. kelleri Forel, 1891, CASENT0466090]. B. Propodeal spines absent; mesonotum $(\mathrm{m})$ greatly raised with respect to pronotum and propodeum [C. tsisitsilo Blaimer, 2013, CASENT0120279]. C. Posterior face of mesonotum (pfm) long; promesonotal suture absent [C. kelleri, CASENT0466090]. D. Promesonotum forming a straight plane, posterior face of mesonotum (pfm) abruptly sloping into metanotal groove, propodeal spines situated high on propodeum (pd) [C. kelleri, CASENT0466090]. E. Pronotum dorsally with distinct median longitudinal groove [C.mafybe sp. nov., CASENT0317762]. F. Pronotum dorsally without median longitudinal groove [C. lobata Emery, 1895, CASENT0125498]. G. Propodeum distinctly set off from promesonotum in lateral view [C. malahelo sp. nov., CASENT0317768]. H. Propodeum not distinctly set off from promesonotum in lateral view [C. dentata Dalla Torre, 1893 stat. nov., CASENT0138289]. 

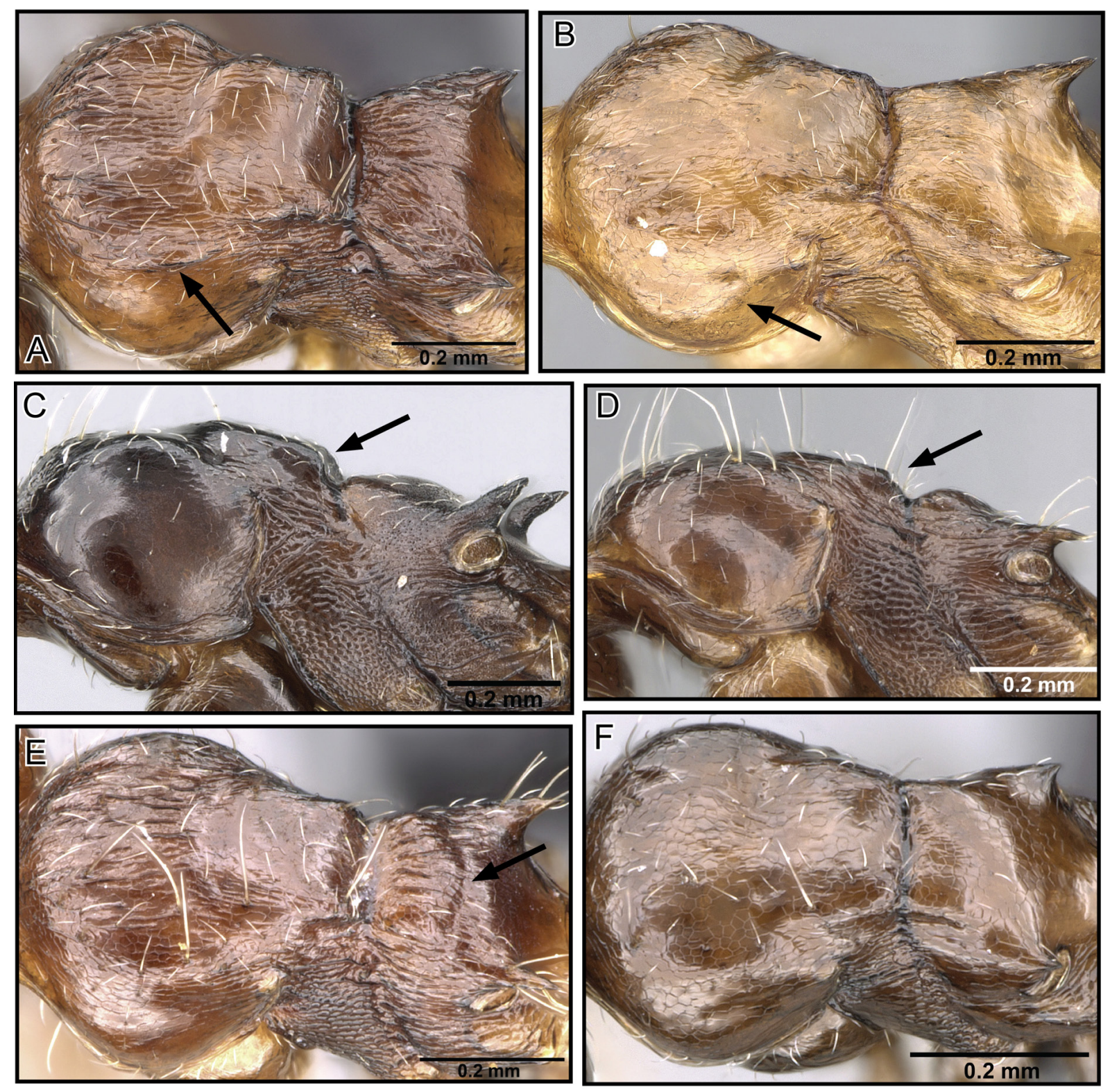

Fig. 5. Key to the species of the C. degeeri-assemblage (part II). A. Pronotum with sharp lateral margins [C. malahelo sp. nov., CASENT0317768]. B. Pronotum lacking sharp lateral margin [C. alafara sp. nov., CASENT0492837]. C. Mesonotum with posterolateral denticles [C. degeeri Forel, 1886, CASENT0071423]. D. Mesonotum without distinct posterolateral denticles [C. ramamy sp. nov., CASENT0317764]. E. Propodeum with distinct short dorsal face and transverse impression, sculpture carinulate [C. ramamy sp. nov., CASENT0317764]. F. Propodeum lacking distinct dorsal face and transverse impression, sculpture rugulose [C. dentata Dalla Torre, 1893 stat. nov., CASENT0138289]. 


\title{
Morphological taxonomy
}

\author{
Class Insecta Linnaeus, 1758 \\ Order Hymenoptera Linnaeus, 1758 \\ Suborder Apocrita Gerstäcker, 1867 \\ Superfamily Vespoidea Latreille, 1802 \\ Family Formicidae Latreille, 1809 \\ Subfamily Myrmicinae Lepeletier de Saint-Fargeau, 1835 \\ Genus Crematogaster Lund, 1831 \\ Crematogaster alafara Blaimer sp. nov. \\ urn:1sid:zoobank.org:act:957DBC9B-0B2D-4ABF-B179-E18F67603927
}

Fig. 6

\section{Diagnosis}

Workers of Crematogaster alafara sp. nov. are fairly small (HW 0.72-0.88, WL 0.79-0.91) and have short to medium-sized propodeal spines (SPI 0.06-0.10). The characteristic promesonotal characters, with the propodeum usually distinctly set off from the promesonotum in lateral view (as Fig. 6B), render this species moderately easy to recognize from other species in the $C$. degeeri-assemblage. Possibly this species could be confused with $C$. malahelo sp. nov.; the latter, however, bears sharp lateral margins on the pronotum (absent in C. alafara sp. nov.) and has shorter, more triangular spines (SPI 0.03-0.07) than C. alafara sp. nov.. Mean genetic distance in the barcode region of $C$. alafara sp. nov. to other species within the $C$. degeeri-assemblage is $15.7-22.2 \%$ (Table 3 ).

\section{Etymology}

The Malagasy word alafara means "last forest", alluding to the restricted distribution of this species as a reminder of forest conservation concerns in Madagascar. This name is treated as a noun in apposition.

\section{Type specimens}

\section{Holotype}

Worker: pinned, CASENT0492837, BLF08619, ex rotten log [imaged on AntWeb]; original locality label: MADG'R: P.N. Ranomafana, Sahamaloatra, 6.6 km 310 NW Ranomafana, 31 Mar. 2003, $21^{\circ} 14.2^{\prime}$ S, $47^{\circ} 23.8^{\prime} \mathrm{E}, 1150 \mathrm{~m}$, Fisher et al., BLF\# (Brian L. Fisher); deposited at CASC.

\section{Paratypes}

4 workers, pinned, \#1 and \#2 same collection and locality data as holotype. \#1: CASENT0317785, deposited at SAMC. \#2: CASENT0317786, deposited at MHNG. \#3 and \#4: BLF08628, ex dead twig above ground, remaining data same as holotype. \#3: CASENT0317787, deposited at MCZC. \#4: CASENT0317788, deposited at UCDC.

\section{Type locality}

MADAGASCAR: Fianarantsoa: P.N. Ranomafana, Sahamaloatra River: -21.23667, 47.39667, 1150 m, montane rainforest.

\section{Other material examined}

(CASC, PSWC): refer to Supplementary Material 1. 


\section{Worker}

\section{Measurements}

$(\mathrm{n}=12)$ [holotype] HW 0.72-0.88 [0.83]; HL 0.69-0.80 [0.78]; EL 0.15-0.19 [0.17]; SL 0.54-0.64 [0.61]; WL 0.79-0.91 [0.90]; SPL 0.05-0.08 [0.06]; PTH 0.17-0.20 [0.20]; PTL 0.22-0.26 [0.26]; PTW 0.290.35 [0.35]; PPL 0.13-0.18 [0.16]; PPW 0.23-0.28 [0.25]; LHT 0.56-0.67 [0.66]; CI 1.02-1.11 [1.06]; OI 0.20-0.25 [0.21]; SI 0.73-0.83 [0.77]; SPI 0.06-0.10 [0.07]; PTHI 0.71-0.81 [0.79]; PTWI 1.18-1.47 [1.36]; PPI 1.56-1.97 [1.59]; LBI 1.30-1.47 [1.38].

\section{Description}

(Fig. 6A-C) Small size (HW 0.72-0.88, WL 0.79-0.91).

Masticatory margin of mandibles with four teeth; head shape quadrate, as long, or slightly longer than wide (CI 1.02-1.11); posterior margin of head in full-face view laterally rounded; occipital carinae distinct; antennal scapes reaching or slightly surpassing head margin; midline of eyes situated slightly above midline of head in full-face view; eyes moderately large (OI 0.20-0.25) and protruding.

Pronotum laterally angular; promesonotal suture indistinct, promesonotum forming one plane; mesonotum usually without posterior face; laterally mesonotum carinate, sometimes ending in small denticles, but not greatly set off from propodeum; metanotal groove shallow, laterally constricted and bordered by carinae; propodeal spines short, spiniform (SPI 0.06-0.10) and straight; dorsal face of propodeum distinct; posterior face of propodeum gently sloping; petiole in dorsal view greatly flared and convex, dorsolaterally rounded to carinate, without posterolateral tubercules or denticles; subpetiolar process usually a small, angular dent; postpetiole distinctly bilobed, with a narrow median impression; subpostpetiolar process absent.

Head sculpture aciculate to faintly reticulate; sculpture otherwise mostly reticulate; face usually with $<$ six erect, longer setae, and regular appressed to decumbent pubescence; erect pilosity on promesonotum usually sparse, < six long setae; otherwise promesonotum dorsally with sparse appressed to subdecumbent pubescence; petiole and postpetiole usually with a pair of short, erect setae posterolaterally, and sparse suberect, decumbent or appressed pubescence; abdominal tergites and sternites four to seven usually with moderately abundant, short, erect pilosity, and regular appressed to decumbent pubescence throughout. Color yellow-brown to brown.

\section{Queen}

\section{Measurements}

$(\mathrm{n}=1) \mathrm{HW} 1.01$, HL 1.01, EL 0.33, SL 0.64, MSNW 0.76, MSNL 0.87, WL 1.68, SPL 0.00, PTH 0.29, PTL 0.33, PTW 0.39, PPL 0.23, PPW 0.39, LHT 0.75, CI 0.99, OI 0.32, SI 0.63, MSNI 0.87, SPI 0.00, PTHI 0.87, PTWI 1.18, PPI 1.68, LBI 2.24.

\section{Description}

(Fig. 6E-G) Small queen (HW 1.01, WL 1.68). With worker characters, except as follows.

Masticatory margin of mandibles with five teeth; antennal scapes reaching level of lateral ocelli; eyes large (OI 0.33), situated below midline of head in full-face view; head elongate-rectangular (CI 0.99), posterior margin straight.

Mesoscutum in dorsal view longer than wide (MSNI 0.87); dorsal face of propodeum short; propodeal tubercules or denticles absent; petiole moderately flared, postpetiole with complete impression; subpetiolar process present as minute tooth. 

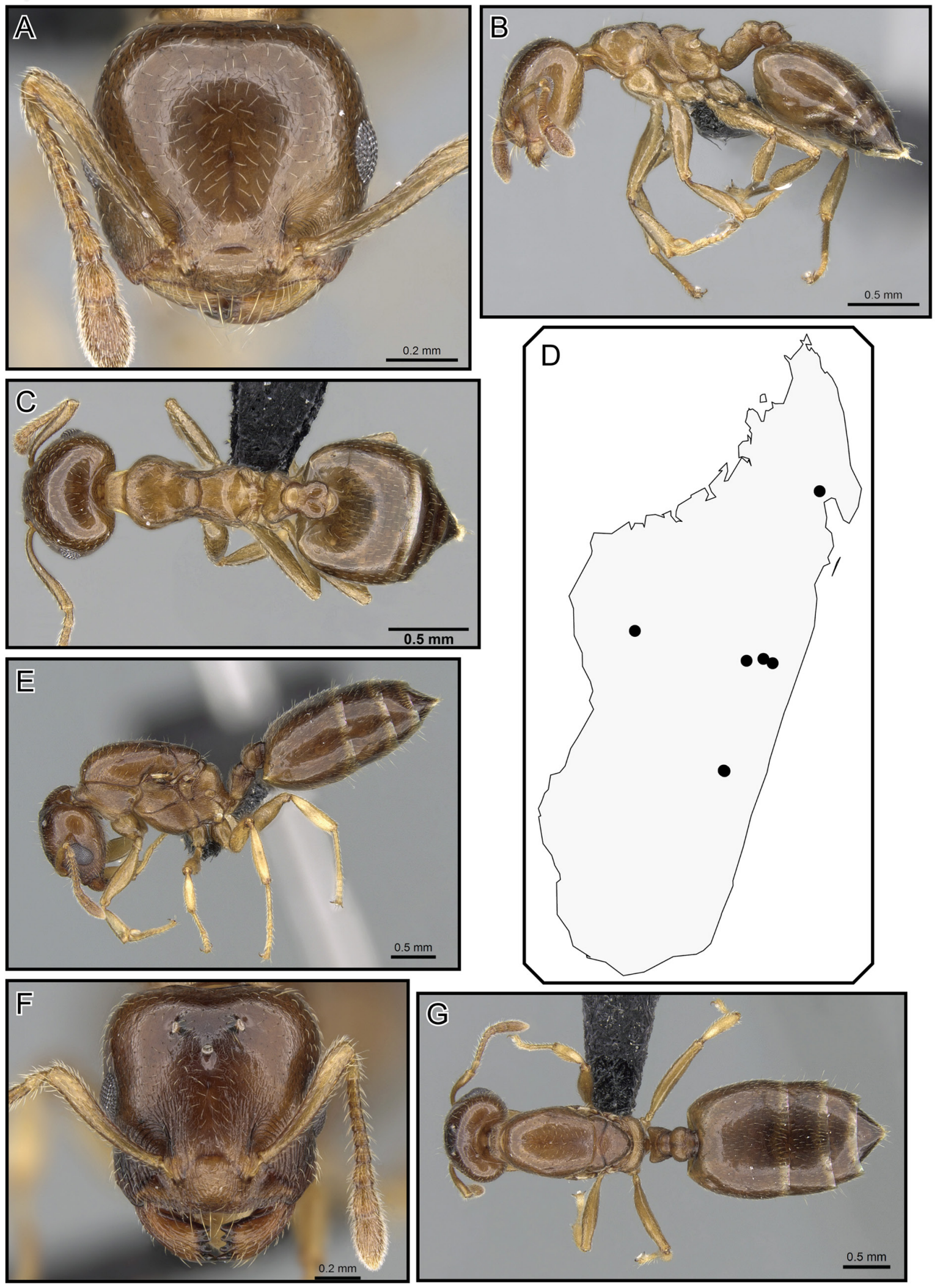

Fig. 6. Crematogaster alafara sp. nov., worker, queen and distribution. A-C. Worker (CASENT0492837). A. Full-face. B. Profile. C. Dorsal. D. Species distribution. E-G. Queen (CASENT0050441). E. Profile. F. Full-face. G. Dorsal. 
Sculpture aciculate to reticulate throughout; face with six longer erect setae (but $n=1$ ) and regular short suberect to decumbent pubescence; mesonotum with regular short, erect to suberect pubescence, interspersed with abundant, longer, erect setae; petiole and postpetiole with fairly dense, short, suberect to decumbent pilosity, and postpetiole with additional longer dorso-posterior setae; abdominal tergites and sternites four to seven with abundant, short, erect pilosity and dense appressed to decumbent pubescence. Color yellowish brown.

\section{Male}

Males of this species are unknown.

\section{Distribution and biology}

MADAGASCAR. Crematogaster alafara sp. nov. has only been collected at a few rainforest localities in central, northern and western Madagascar (Fig. 6D) ranging from $600 \mathrm{~m}$ to $1350 \mathrm{~m}$ in elevation. The species is known to co-occur with $C$. sewellii, $C$. dentata stat. nov. and C. mafybe sp. nov.. The few collections have been made from dead twigs above ground at the lower elevation range, and from rotten logs at higher altitudes. Otherwise nothing is known of the biology of this species.

Crematogaster bara Blaimer sp. nov. urn:1sid:zoobank.org:act:CD497C84-8C12-4005-9817-5236FA0AC4C2

Fig. 7

\section{Diagnosis}

Crematogaster bara sp. nov. is easily distinguished from all other species of the $C$. degeeri-assemblage by a combination of the following characters: yellow body color, medium to large size (HW 0.87-1.03, WL 0.98-1.22) and very long antennal scapes (SI 0.91-1.01). Mean barcode divergence between C. bara sp. nov. and the remaining species is $7.9-18.0 \%$ (Table 3 ).

\section{Etymology}

Crematogaster bara sp. nov. is named after the Malagasy "Bara" tribe that inhabits the countryside within the distribution range of this species. The name is treated as noun in apposition.

\section{Type specimens}

\section{Holotype}

Worker: pinned, CASENT0491123, BLF07783, under stone [imaged on AntWeb]; original locality label: MADG'R: Fianarantsoa: P.N. Isalo, Ambovo Springs, $29.3 \mathrm{~km} 4^{\circ} \mathrm{N}$ Ranohira $990 \mathrm{~m}, 22^{\circ} 19.9^{\prime}$ S, 4521.1'E, 14 Feb. 2003, Fisher et al., BLF\#; deposited at CASC.

\section{Paratypes}

4 workers, pinned, same collection and locality data as holotype. \#1: CASENT0317776, deposited at SAMC. \#2: CASENT0317777, deposited at MHNG. \#3: CASENT0317778, deposited at MCZC. \#4: CASENT0317779, deposited at UCDC.

\section{Type locality}

MADAGASCAR: Fianarantsoa: P.N. Isalo, Ambovo Springs, $29.3 \mathrm{~km} 4^{\circ} \mathrm{N}$ Ranohira, -22.29833, 45.35167, 990 m, Uapaca woodland.

\section{Other material examined}

(CASC): refer to Supplementary Material 1. 


\section{Worker}

\section{Measurements}

$(\mathrm{n}=16)$ [holotype] HW 0.87-1.03 [0.96]; HL 0.78-0.95 [0.89]; EL 0.18-0.23 [0.20]; SL 0.77-0.87 [0.83]; WL 0.98-1.22 [1.07]; SPL 0.10-0.18 [0.12]; PTH 0.18-0.24 [0.20]; PTL 0.27-0.34 [0.30]; PTW 0.280.36 [0.31]; PPL 0.15-0.19 [0.16]; PPW 0.28-0.35 [0.29]; LHT 0.77-0.93 [0.82]; CI 1.04-1.14 [1.08]; OI 0.21-0.25 [0.22]; SI 0.91-1.01 [0.93]; SPI 0.09-0.17 [0.12]; PTHI 0.62-0.75 [0.68]; PTWI 0.97-1.17 [1.02]; PPI 1.65-2.00 [1.86]; LBI 1.20-1.32 [1.29].

\section{Description}

(Fig. 7A-C) Medium to large size (HW 0.87-1.03, WL 0.98-1.22).

Masticatory margin of mandibles with four teeth; head shape quadrate, or longer than wide (CI 1.041.14); posterior margin of head in full-face view laterally rounded or subangular; occipital carinae usually distinct; antennal scapes always easily surpassing head margin; midline of eyes situated at midline of head in full-face view; eyes moderately large (OI 0.21-0.25) and protruding.

Pronotum laterally subangular to rounded; promesonotal suture indistinct; promesonotum more or less forming one rounded plane, mesonotum usually with a short posterior face; laterally mesonotum angular, ending in minuscule posterolateral denticles that set off the promesonotum from propodeum; metanotal groove shallow, laterally constricted; propodeal spines short, spiniform (SPI 0.09-0.17); propodeal spiracles large, situated directly at base of spines; dorsal face of propodeum convex, almost as long as posterior face; posterior face of propodeum gently sloping; petiole in dorsal view broadly oval or moderately flared, usually concave, without posterolateral tubercules or denticles; subpetiolar process usually present as angular dent; postpetiole very distinctly bilobed, with broad median impression; subpostpetiolar process absent.

Head sculpture reticulate; pronotum dorsally rugulose-reticulate; mesonotum reticulate, mesopleuron areolate, dorsal face of propodeum reticulate-carinulate; otherwise sculpture mostly reticulate; face usually with $<$ six erect, longer setae, and regular, shorter, appressed to decumbent pubescence; erect pilosity on promesonotum usually < eight long setae; otherwise promesonotum dorsally with regular appressed pubescence; petiole and postpetiole usually with a pair of longer erect setae posterolaterally, and shorter decumbent or appressed pubescence; abdominal tergites four to seven with fairly regular, short, erect pilosity, more abundant and longer on sternites four to seven, and abundant appressed pubescence throughout. Color bright to reddish yellow.

\section{Queen}

\section{Measurements}

$(\mathrm{n}=3)$ HW 1.42-1.45; HL 1.32-1.37; EL 0.39-0.40; SL 1.03-1.12; MSNW 1.22-1.48; MSNL 1.37-1.44; WL 2.46-2.75; SPL 0.00; PTH 0.37-0.40; PTL 0.44-0.47; PTW 0.55-0.61; PPL 0.37-0.38; PPW 0.590.61; LHT 1.22-1.25; CI 1.06-1.08; OI 0.28-0.31; SI 0.76-0.82; MSNI 0.89-1.07; SPI 0.00; PTHI 0.840.87; PTWI 1.23-1.31; PPI 1.61; LBI 2.12-2.20.

\section{Description}

(Fig. 7E-G) Medium size (HW 1.42-1.45, WL 2.46-2.75). With worker characters, except as follows.

Masticatory margin of mandibles with five teeth; antennal scapes easily surpassing level of lateral ocelli; eyes fairly large (OI 0.28-0.31), situated at midline of head in full-face view; head shape more or less quadrate (CI 1.06-1.08), posterior margin straight. 

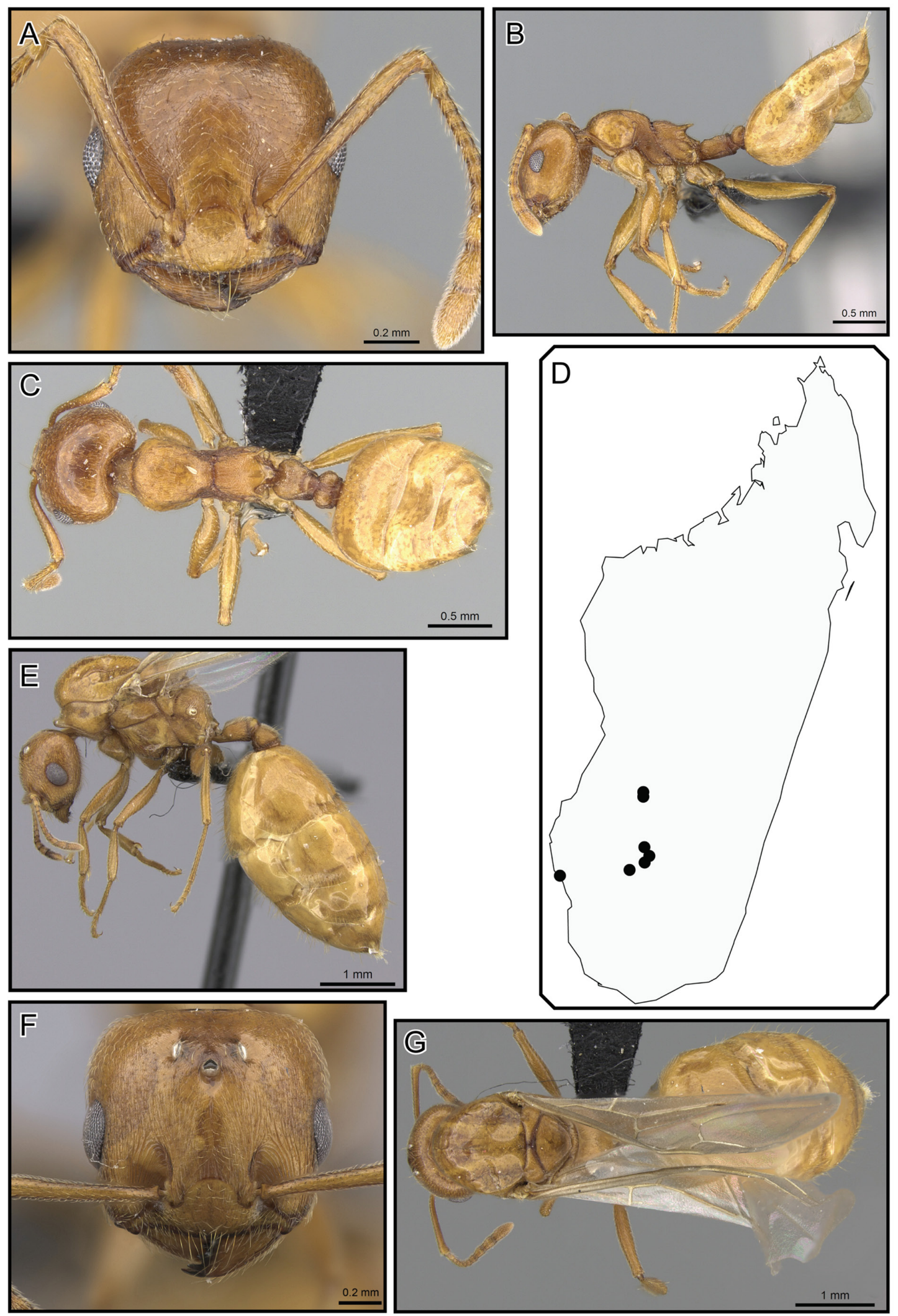

Fig. 7. Crematogaster bara sp. nov., worker, queen and distribution. A-C. Worker (CASENT0491123). A. Full-face. B. Profile. C. Dorsal. D. Species distribution. E-G. Queen (CASENT0064664). E. Profile. F. Full-face. G. Dorsal. 
Mesoscutum in dorsal view variable (MSNI 0.89-1.07); dorsal face of propodeum short; propodeal tubercules or denticles may be present; petiole moderately flared, postpetiole merely impressed posteriorly; subpetiolar process absent.

Head sculpture reticulate; dorsal face of propodeum carinulate; petiole and postpetiole reticulate; otherwise sculpture reticulate throughout; face usually with $<$ ten longer, erect setae and dense, short, erect to decumbent pubescence; mesonotum with regular, short, suberect pubescence, interspersed with longer, erect setae; petiole and postpetiole with dense, short, suberect to decumbent pilosity, and postpetiole with additional, longer dorso-posterior setae; abdominal tergites and sternites four to seven with abundant, short, erect pilosity and dense appressed to decumbent pubescence. Colored yellow as in workers; wings clear.

\section{Male}

Males of this species are unknown.

\section{Distribution and biology}

MADAGASCAR. Crematogaster bara sp. nov. has a very narrow distribution range in gallery forest, savannah, Uapaca woodland and spiny forest habitat in southwestern Madagascar (Fig. 7D). It has been collected at elevations from $30 \mathrm{~m}$ to $1100 \mathrm{~m}$, and occurs in sympatry with $C$. sewellii, C. dentata stat. nov. and $C$. maina sp. nov. Not much is known about the biology of this species. One collection has been made of a colony nesting under a stone, indicating that this species may be ground-nesting.

\section{Crematogaster degeeri Forel, 1886}

Figs 8-9

Crematogaster degeeri Forel, 1886: cvii.

Crematogaster (Acrocoelia) degeeri var. lunaris Santschi, 1928: 64. Worker from MADAGASCAR: Mont. Voiposa, Ambohimahosoa (Descarpentries, leg.) [NHMB, examined]. Lectotype worker by present designation [CASENT0101174, image on AntWeb]. syn. nov.

Crematogaster degeeri - Forel 1891: 190 (queen and male described).

Crematogaster geeri - Schulz 1906: 155; unjustified subsequent emendation.

Crematogaster (Crematogaster) degeeri - Wheeler 1922b: 1022. — Bolton 1995: 166.

Crematogaster (Acrocoelia) degeeri - Emery 1922: 146.

\section{Diagnosis}

Within the $C$. degeeri-assemblage, this species is most easily recognized by its large eyes (OI $0.22-$ 0.26 ), fairly long propodeal spines (SPI 0.10-0.16), and a promesonotum that usually bears angular posterolateral denticles (Fig. 5C) and is distinctly set off from propodeum in lateral view. Crematogaster degeeri could be confused with $C$. ramamy sp. nov. and $C$. dentata stat. nov., but in both of the latter the promesonotum is not distinctly set off from the propodeum. Furthermore, the latter two species have generally shorter propodeal spines $(C$. dentata stat. nov. SPI 0.01-0.10; C. ramamy sp. nov. SPI 0.07$0.12)$.

Mean barcode divergences between $C$. degeeri and species within the $C$. degeeri-assemblage are 13.2$18.0 \%$, except with regard to $C$. ramamy, where this level is only $4.7 \%$ (Table 3 ). In our analysis, $C$. degeeri is paraphyletic with respect to C. ramamy (see comments below). 


\section{Type material examined}

(MHNG) MADAGASCAR (M. Grandidier), 2 worker syntypes. Lectotype worker by present designation: top worker of these two specimens on one pin [CASENT0101690; imaged on AntWeb].

\section{Other material examined}

(CASC, MCZC, MHNG, NMBH, PSWC): refer to Supplementary Material 1.

\section{Worker}

\section{Measurements}

( $\mathrm{n}=15)$ HW 0.81-1.05; HL 0.76-0.97; EL 0.18-0.24; SL 0.63-0.78; WL 0.81-1.09; SPL 0.08-0.16; PTH 0.17-0.22; PTL 0.25-0.33; PTW 0.28-0.41; PPL 0.14-0.20; PPW 0.24-0.33; LHT 0.60-0.81; CI 1.061.11; OI 0.22-0.26; SI 0.77-0.86; SPI 0.10-0.16; PTHI 0.56-0.77; PTWI 1.01-1.39; PPI 1.52-1.95; LBI $1.29-1.44$.

\section{Description}

(Fig. 8A-C) Small to medium size (HW 0.81-1.05, WL 0.81-1.09).

Masticatory margin of mandibles with four teeth; head shape quadrate, mostly as long as wide (CI 1.06-1.11); posterior margin of head in full-face view laterally rounded or subangular; occipital carinae usually distinct; antennal scapes reaching or surpassing head margin; midline of eyes situated slightly above midline of head in full-face view; eyes fairly large (OI 0.22-0.26) and moderately protruding.

Pronotum laterally angular; promesonotal suture indistinct; mesonotum more or less forming one plane with pronotum, but often the two separated by distinct variation in sculpture (see below); mesonotum usually with a short posterior face; laterally mesonotum angular to carinate, and often with posterolateral denticles, which set off the promesonotum distinctly from propodeum; posterior face of mesonotum longitudinally depressed with respect to lateral carinae, slopes gently into metanotal groove; metanotal groove fairly shallow, laterally constricted; propodeal spines short, spiniform (SPI 0.10-0.16), usually straight and moderately diverging; propodeal spiracles large, situated directly at base of spines; dorsal face of propodeum convex, almost as long as posterior face; posterior face of propodeum gently sloping; petiole in dorsal view from moderately to strongly flared and usually strongly concave, dorsolaterally carinate but without posterolateral tubercules or denticles; subpetiolar process variable; postpetiole wider than long, very distinctly bilobed by a sharp median impression; subpostpetiolar process absent.

Head sculpture shiny to aciculate; pronotum usually dorsally costulate and contrasting abruptly with a shiny to aciculate mesonotum; propleuron aciculate or reticulate, mesopleuron areolate, metapleuron mostly carinulate; dorsal face of propodeum carinulate or reticulate, posterior face shiny; dorsal face of petiole reticulate; helcium reticulate; postpetiole dorsally aciculate-reticulate; lateral and ventral face of petiole and postpetiole reticulate; face usually with four to eight erect, longer setae, and regular shorter appressed to suberect pubescence; erect pilosity on promesonotum variable, usually < eight long setae; otherwise promesonotum dorsally with regular appressed to subdecumbent pubescence; petiole and postpetiole usually with a pair of short erect setae posterolaterally, and shorter suberect, decumbent or appressed pubescence; abdominal tergites four to seven with very sparse, short, erect pilosity, more abundant and longer on sternites four to seven, and abundant appressed to decumbent pubescence throughout. Color reddish brown to dark brown or black.

\section{Queen}

\section{Measurements}

$(\mathrm{n}=3)$ HW 1.44-1.56; HL 1.32-1.42; EL 0.40-0.45; SL 0.95-0.99; MSNW 1.20-1.46; MSNL 1.42-1.50; WL 2.49-2.68; SPL 0.00; PTH 0.38-0.41; PTL 0.43-0.53; PTW 0.51-0.59; PPL 0.35-0.38; PPW 0.56- 
0.60; LHT 1.12-1.14; CI 1.09-1.10; OI 0.28-0.32; SI 0.68-0.72; MSNI 0.83-0.97; SPI 0.00; PTHI 0.720.95; PTWI 1.06-1.27; PPI 1.53-1.60; LBI 2.23-2.37.

\section{Description}

(Fig. 8D-F) Medium size (HW 1.44-1.56, WL 2.49-2.68). With worker characters, except as follows.

Masticatory margin of mandibles with five teeth. Antennal scapes not reaching posterior margin of head, but surpassing level of lateral ocelli; eyes large (OI 0.28-0.32), situated at midline of head in full-face view; head shape fairly quadrate (CI 1.09-1.10), moderately tapering from posterior to anterior margin; posterior margin straight.
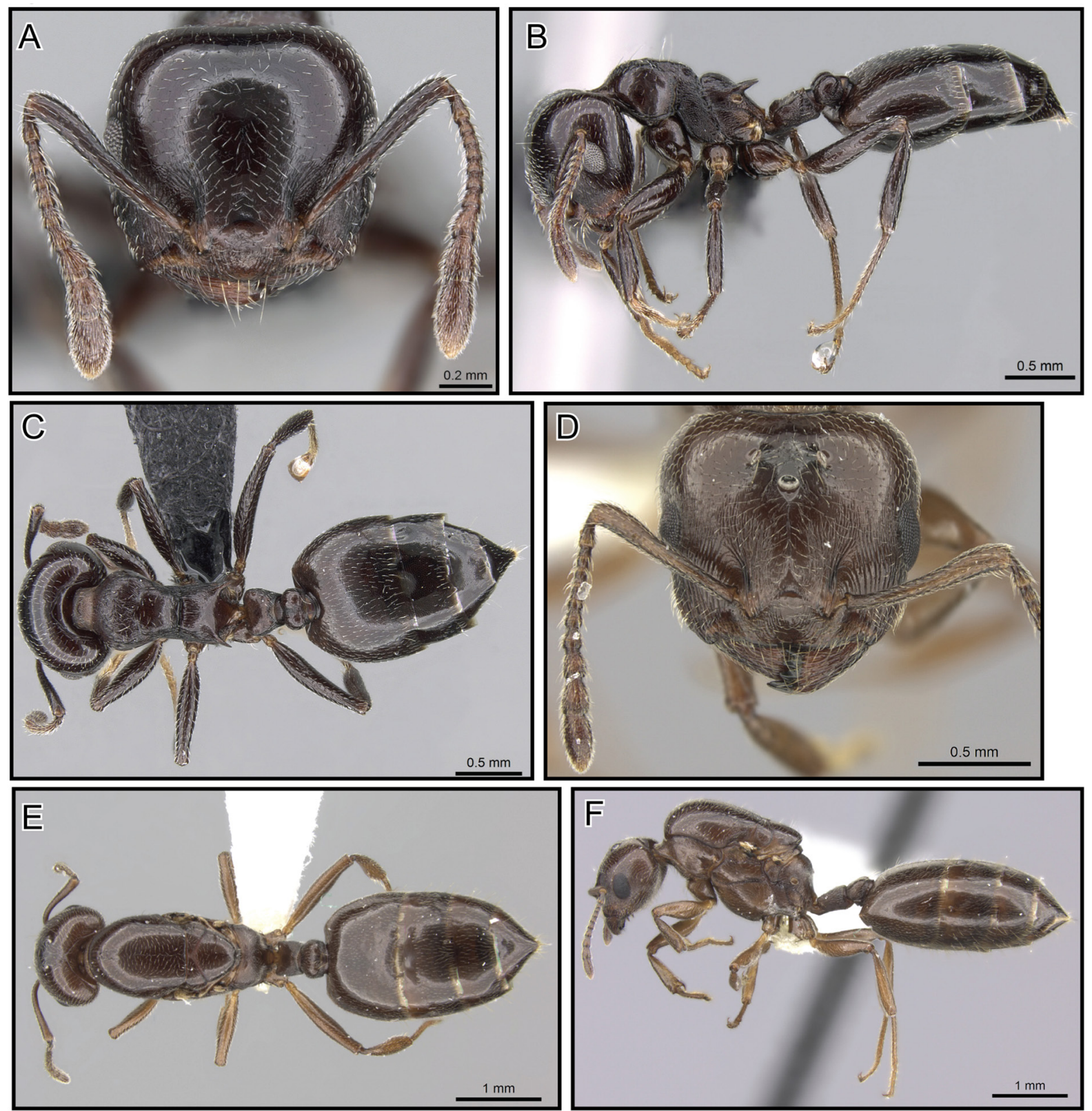

Fig. 8. Crematogaster degeeri Forel, 1886, worker and queen. A-C. Worker (CASENT0118982). A. Full-face. B. Profile. C. Dorsal. D-F. Queen (CASENT0012763). D. Full-face. E. Dorsal F. Profile. 
Mesosoma fairly compact (MSNI 0.83-0.97, WL 2.49-2.68); mesoscutum in dorsal view about as wide as long; dorsal face of propodeum distinct but short; propodeum tuberculate but distinct spines absent; petiole moderately flared, postpetiole merely impressed behind; subpetiolar process variable.

Sculpture shiny to aciculate throughout; face with four to six longer erect setae and abundant shorter erect to suberect pilosity; mesonotum with abundant, short, and scattered longer erect setae; petiole and postpetiole with very dense short suberect pilosity, and postpetiole often with additional longer dorsoposterior setae; abdominal tergites and four to seven with abundant appressed pilosity and regular, short, erect pilosity. Color similar to respective workers; wings clear.

\section{Male}

\section{Measurements}

$(\mathrm{n}=1)$ HW 0.74; HL 0.51; EL 0.29; SL 0.12; MSNW 0.91; MSNL 0.82; WL 1.53; SPL 0.00; PTH 0.24; PTL 0.26; PTW 0.30; PPL 0.19; PPW 0.35; LHT 0.86; CI 1.46; OI 0.57; SI 0.24; MSNI 1.12; SPI 0.00; PTHI 0.91; PTWI 1.16; PPI 1.82; LBI 1.78.

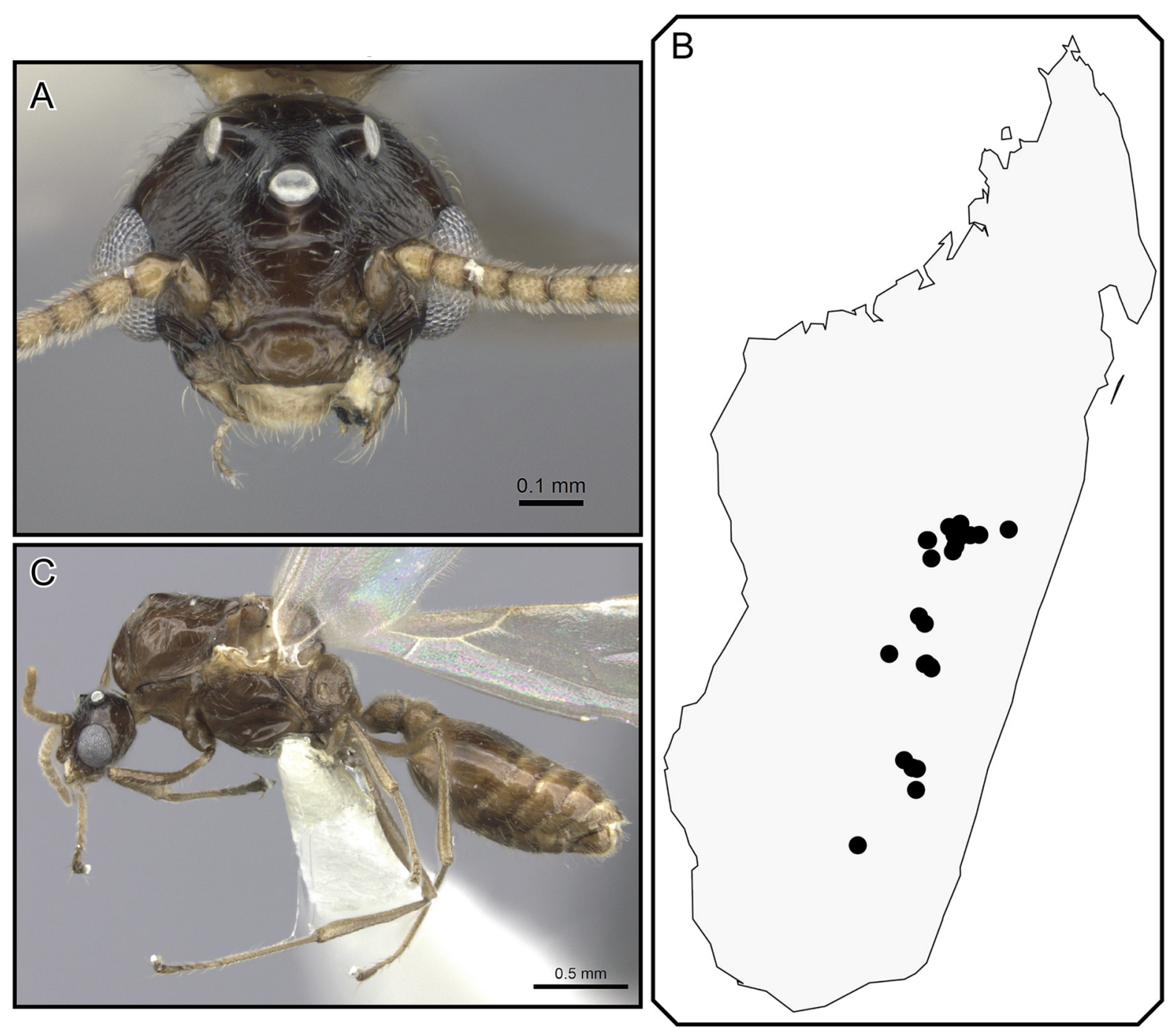

Fig. 9. Crematogaster degeeri Forel, 1886, male and distribution. A, C. (CASENT0127520). A. Fullface. C. Profile. B. Species distribution. 


\section{Description}

(Fig. 9A, C) Small (HW 0.74, WL 1.53).

Mandibles very short and reduced, masticatory margin with two teeth, one large apical and a minute second; clypeus moderately protruding; eyes large (OI 0.57 ) and protruding, midline of eyes situated well below midline of head, almost extending to clypeal margin; antennae 12-segmented; head much wider than long (CI 1.46); in full-face view ocellar triangle situated at posterior head margin and elevated with respect to rest of face; occipital carinae distinct.

Mesoscutum in dorsal view as wide as long (MSNI 1.12); scutellum in dorsal view broadly tapering from anterior to posterior end, dorsoposteriorly rounded; metanotum protruding from below scutellum; dorsal face of propodeum almost as long as posterior face; propodeal spines absent; petiole in dorsal view oval, laterally rounded, in lateral view anteriorly greatly tapering; subpetiolar process absent; postpetiole round, lacking median impression.

Head sculpture shiny to rugulose, clypeus shiny; mesoscutum with very fine longitudinal carinulae; otherwise sculpture more or less shiny to aciculate; scattered, short, erect pilosity and a few longer setae on face, mesoscutum, and scutellum; remaining pilosity as in queens. Color brown.

\section{Distribution and biology}

MADAGASCAR. Crematogaster degeeri occurs in montane rainforest, savannah, woodland and grassland habitats in central and south-central Madagascar at altitudes from 800 to $2650 \mathrm{~m}$ (Fig. 9B). This species occurs often in sympatry with C. mafybe sp. nov., and at some locations with C. sewelli. These ants are often found nesting on the ground under stones or in the soil, although a few records of arboreal twig collections also exist.

\section{Comments}

In the molecular analysis, Crematogaster degeeri was found to be not reciprocally monophyletic with respect to C. ramamy (see above and Fig. 1). Refer to comments under C. ramamy for further discussion.

Crematogaster dentata Dalla Torre, 1893 stat. nov.

Figs 10-11

Crematogaster sewellei var. dentata Dalla Torre, 1893: 86.

Crematogaster sewelli var. improba Forel, 1907: 80 (footnote). Worker syntypes from MADAGASCAR: Morondava (collector unknown) [MHNG, examined]. Lectotype worker by present designation: CASENT0101677, image on AntWeb, middle w of 3w specimens on one pin. syn. nov.

Crematogaster sewelli mauritiana Forel, 1907: 79; worker syntypes from MAURITIUS (Voeltzkow) [MHNG, examined]. Lectotype worker by present designation: CASENT0101752, image on AntWeb. syn. nov.

Crematogaster inermis r. sewellii var. dentatus - Forel 1891: 196; unavailable name.

Crematogaster sewellei dentata - Forel 1907: 79. - Donisthorpe 1946: 30.

C. (Acrocoelia) sewellei var. dentata - Emery 1922: 148.

C. (Acrocoelia) sewelli var. improba - Emery 1922: 148.

C. (Acrocoelia) sewelli mauritiana - Emery 1922: 148.

\section{Diagnosis}

Crematogaster dentata stat. nov. is a highly variable, widespread species and thus very difficult to diagnose based upon a single or even a few characters. Workers can be very small to medium size (HW 
0.72-1.10, WL 0.74-1.11), but usually have very abundant pilosity on the promesonotum ( $>$ eight setae) and sculpture is mostly aciculate to reticulate throughout. This species is most likely to be confused with C. degeeri and C. ramamy sp. nov.. Crematogaster degeeri, however, has a promesonotum very distinctly set off from the propodeum and bears posterolateral denticles on the mesonotum, whereas typically in $C$. dentata stat. nov. the transition between mesonotum and propodeum is fluent and the metanotal groove shallow. Crematogaster ramamy sp. nov. always shows costulate sculpture on the pronotum, whereas in $C$. dentata the sculpture on the pronotum is reduced-aciculate or reticulate. Genetic divergence in the barcode region of this species with respect to other taxa within the $C$. degeeri-assemblage ranges from $12.7-21.3 \%$ in our analysis (Table 3 ).

\section{Type material examined}

(MHNG): MADAGASCAR (M. Grandidier), 1 worker, holotype by monotypy [CASENT0101683; imaged on AntWeb].

\section{Other material examined}

(BBBC, CASC, MHNG, NHMB, PSWC, ZMHB): refer to Supplementary Material 1.

\section{Worker}

\section{Measurements}

$(\mathrm{n}=31)$ HW 0.72-1.10; HL 0.70-1.03; EL 0.13-0.24; SL 0.52-0.78; WL 0.74-1.11; SPL 0.01-0.10; PTH 0.12-0.24; PTL 0.23-0.35; PTW 0.24-0.41; PPL 0.11-0.21; PPW 0.21-0.38; LHT 0.55-0.84; CI 1.011.11; OI 0.17-0.26; SI 0.69-0.82; SPI 0.01-0.10; PTHI 0.52-0.77; PTWI 0.95-1.31; PPI 1.51-2.02; LBI 1.26-1.46.

\section{Description}

(Fig. 10A-F) Very small to medium-sized (HW 0.72-1.10, WL 0.74-1.11).

Masticatory margin of mandibles with four teeth; head shape quadrate, mostly as long as wide (CI 1.01-1.11); posterior margin of head in full-face view laterally rounded or subangular; occipital carinae usually distinct; antennal scapes variable, but often not reaching head margin; midline of eyes situated at midline of head in full-face view; eye size fairly variable (OI $0.17-0.26$ ), usually only slightly protruding.

Pronotum laterally subangular; promesonotal suture indistinct, mesonotum more or less forming one plane with pronotum; mesonotum usually without a distinct posterior face; laterally mesonotum angular and often with posterolateral tubercules or denticles; mesonotum longitudinally depressed with respect to lateral borders, sloping gently into metanotal groove; metanotal groove more or less absent; propodeal spines highly variable, from reduced minute denticles to short and spiniform (SPI 0.01-0.10), if spiniform then often downcurved; propodeal spiracles large, situated directly at base of spines; dorsal face of propodeum variable, from almost as long as posterior face to nearly absent; posterior face of propodeum from gently to steeply sloping; petiole in dorsal view oval, or moderately to strongly flared, moderately to strongly concave, usually not dorsolaterally carinate and without posterolateral tubercules or denticles; subpetiolar process variable; postpetiole wider than long, distinctly bilobed with a broad median impression; subpostpetiolar process absent.

Head sculpture aciculate; promesonotum usually dorsally reticulate or aciculate, rarely pronotum carinulate; propleuron aciculate, mesopleuron areolate, metapleuron mostly reticulate; dorsal face of propodeum reticulate or aciculate, posterior face shiny; dorsal face of petiole reticulate to shiny; helcium reticulate; postpetiole dorsally aciculate; lateral and ventral face of petiole and postpetiole reticulate; face usually with four to eight erect, longer setae, and regular, shorter, appressed to suberect pubescence; erect pilosity on promesonotum variable, but usually very abundant $>$ eight long setae; otherwise promesonotum 
BLAIMER B. \& FISHER B.L., The Crematogaster degeeri-species-assemblage
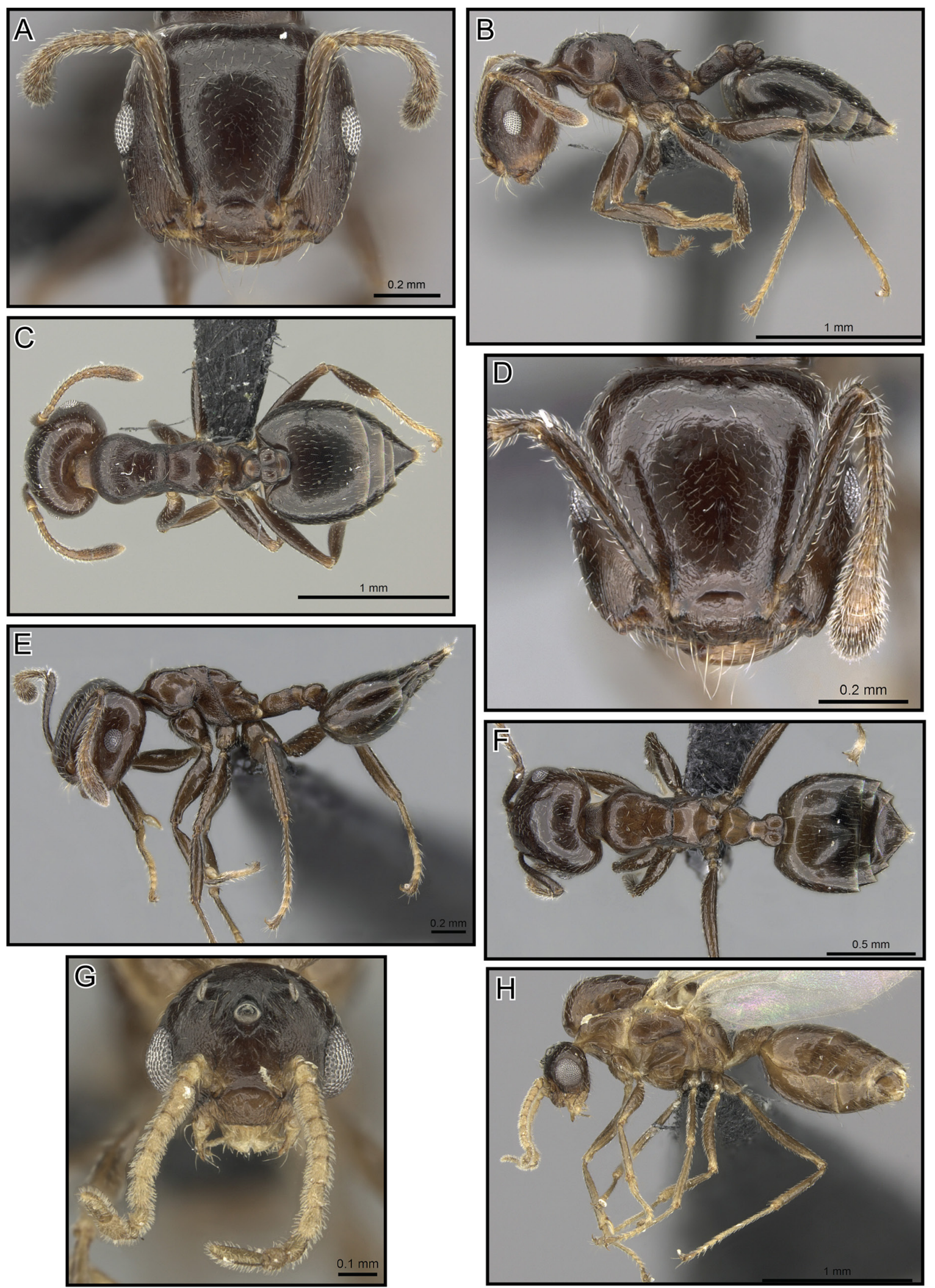

Fig. 10. Crematogaster dentata Dalla Torre, 1893 stat. nov., workers and male. A-C. Worker (CASENT0456641). A. Full-face. B. Profile. C. Dorsal. D-F. Worker (CASENT0138289). D. Full-face E. Profile F. Dorsal. G-H. Male (CASENT0456644). G. Full-face. H. Profile. 
dorsally with regular to sparse appressed to subdecumbent pubescence; petiole and postpetiole usually with a pair of longer erect setae posterolaterally, and shorter suberect, decumbent or appressed pubescence; abdominal tergites four to seven with moderately abundant, short, erect pilosity, and regular appressed to decumbent pubescence throughout. Color light brown to dark brown, black, orange-red with black abdominal segment four to seven (western and southern dry and spiny forest localities).

\section{Queen}

\section{Measurements}

$(\mathrm{n}=5)$ HW 1.13-1.41; HL 1.17-1.46; EL 0.30-0.42; SL 0.67-0.88; MSNW 0.92-1.19; MSNL 1.00-1.30; WL 1.73-2.43; SPL 0.00; PTH 0.30-0.38; PTL 0.35-0.43; PTW 0.41-0.52; PPL 0.26-0.39; PPW 0.400.55; LHT 0.80-1.05; CI 0.95-1.03; OI 0.25-0.31; SI 0.57-0.67; MSNI 0.86-0.92; SPI 0.00; PTHI 0.760.90; PTWI 1.10-1.21; PPI 1.39-1.68; LBI 2.17-2.32.

\section{Description}

(Fig. 11A-C) Small to medium size (HW 1.13-1.41, WL 1.73-2.43). With worker characters, except as follows.

Masticatory margin of mandibles with five teeth. Antennal scapes very short, often not reaching level of lateral ocelli; eyes fairly large (OI 0.25-0.31), situated distinctly below midline of head in full-face view; head shape usually rectangular, elongate, longer than wide (CI 0.95-1.03); posterior margin straight.

Mesoscutum in dorsal view longer than wide (MSNI 0.86-0.92); dorsal face of propodeum distinct but short; propodeal spines or tubercules absent; petiole moderately flared, postpetiole merely impressed behind; subpetiolar process variable.

Sculpture shiny to aciculate throughout; face usually with $>$ six longer erect setae and abundant, shorter, erect to suberect pilosity; mesonotum with abundant short, and scattered longer, erect setae; petiole and postpetiole with dense short suberect pilosity, and postpetiole often with additional longer dorsoposterior setae; abdominal tergites and sternites four to seven with abundant appressed pilosity and sparse, short, erect pilosity. Color similar to respective workers; wings clear.

\section{Male}

\section{Measurements}

$(\mathrm{n}=3)$ HW 0.56-0.64; HL 0.42-0.48; EL 0.21-0.23; SL 0.10-0.13; MSNW 0.65-0.74; MSNL 0.50-0.69; WL 1.09-1.40; SPL 0.00; PTH 0.16-0.17; PTL 0.20-0.24; PTW 0.22-0.27; PPL 0.12-0.14; PPW 0.240.27; LHT 0.54-0.62; CI 1.32-1.35; OI 0.47-0.53; SI 0.23-0.27; MSNI 1.08-1.45; SPI 0.00; PTHI 0.710.83; PTWI 0.97-1.24; PPI 1.74-2.16; LBI 2.01-2.25.

\section{Description}

(Fig. 10G-H) Small (HW 0.56-0.64, WL 1.09-1.40).

Mandibles short and reduced, blunt and edentate; clypeus moderately protruding; eyes moderately sized (OI 0.47-0.53) and protruding, midline of eyes situated well below midline of head, almost extending to clypeal margin; antennae 12-segmented; head wider than long (CI 1.32-1.35); in full-face view ocellar triangle situated at posterior head margin, but not much elevated with respect to rest of face; occipital carinae distinct.

Mesoscutum in dorsal view as wide as long or wider than long (MSNI 1.08-1.45); scutellum in dorsal view broadly tapering from anterior to posterior end, dorsoposterior part rounded; metanotum protruding from below scutellum; dorsal face of propodeum almost as long as posterior face; propodeal 
spines absent; petiole in dorsal view oval, laterally rounded, in lateral view greatly tapering anteriorly; subpetiolar process absent; postpetiole fairly flat, lacking median impression.

Head sculpture, including clypeus, shiny to rugulose; mesoscutum with very fine longitudinal carinulae; otherwise sculpture more or less shiny to aciculate; scattered short, erect pilosity and a few longer setae on face, mesoscutum, and scutellum; remaining pilosity as in queens. Color brown.
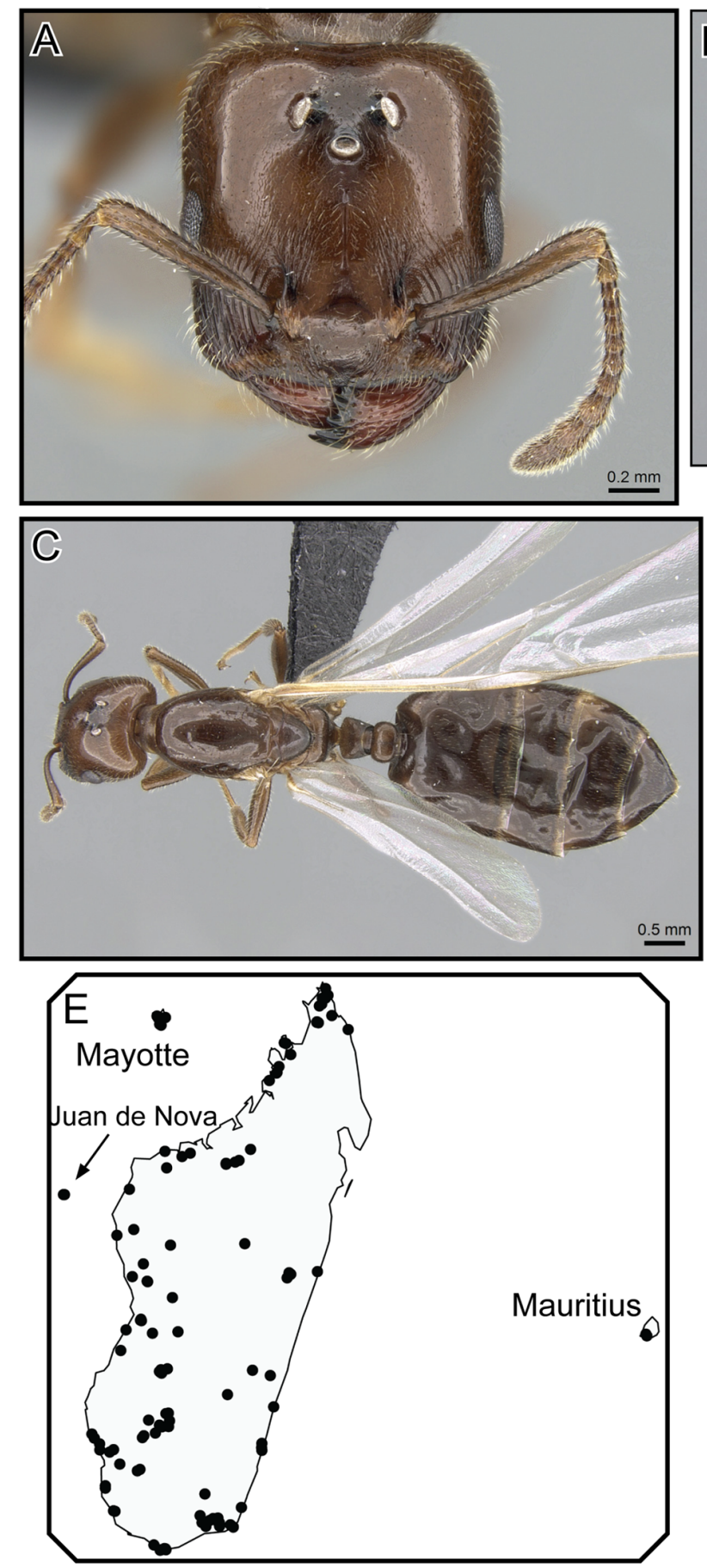
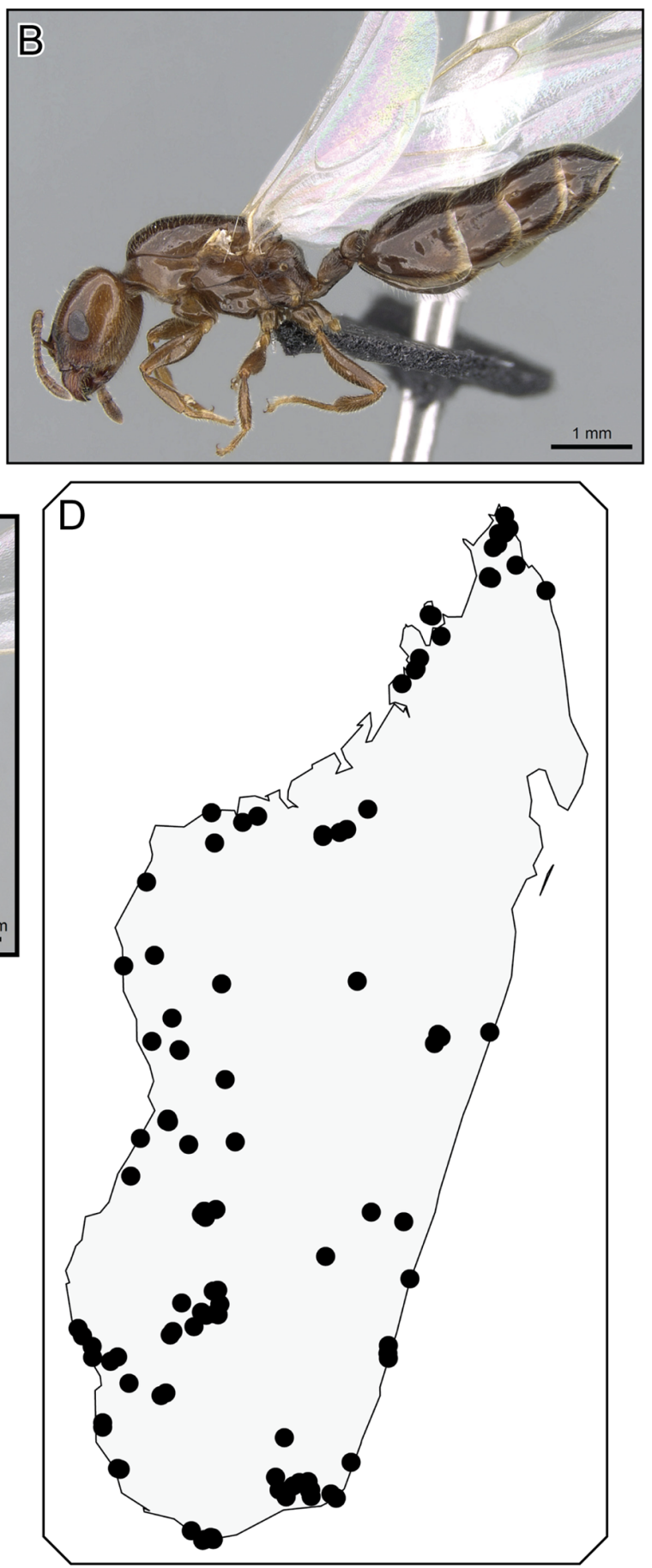

Fig. 11. Crematogaster dentata Dalla Torre, 1893 stat. nov., queen and distribution. A-C. Queen (CASENT0484998). A. Full-face. B. Profile. C. Dorsal. D-E. Species distribution. 


\section{Distribution and biology}

MADAGASCAR, MAURITIUS, MAYOTTE and JUAN DE NOVA. Crematogaster dentata stat. nov. is the most widespread species within the $C$. degeeri-assemblage in the Malagasy region (Fig. 11D-E). The species occurs in dry deciduous and littoral forests of western, southwestern and northern Madagascar, in the spiny forest of southern Madagascar and in coastal shrub and mangrove habitats. A few records exist from rainforest sites, but the species is clearly adapted to more mesic habitats. Crematogaster dentata stat. nov. is thus most common at low elevations, but its elevational distribution range seems to be quite broad, with a maximum record of $1410 \mathrm{~m}$ in transition forest in Ambohitantely Reserve. On Mayotte and Juan de Nova it has been found to occupy coastal shrub habitats, whereas on Mauritius it is known to occur in rainforest habitat. Given its broad distribution range, this species co-occurs with most other species in the $C$. degeeri-assemblage, with the exception of the species generally adapted to higher elevations (C. alafara sp. nov., C. degeeri, C. malahelo sp. nov.). Crematogaster dentata stat. nov. is most often found nesting arboreally in dead twigs and branches, and also in live plant parts. In baiting surveys in western Madagascar this species was active and attracted to fish baits during both day and night.

\section{Comments}

Crematogaster dentata stat. nov. is morphologically the most variable species within the $C$. degeeri -assemblage. Workers show a wide range of gradual variation in size and mesosomal structure. The variation in mesosomal characters in particular can lead to confusion with other species, making $C$. dentata stat. nov. difficult to diagnose. A general trend seems to be that workers from populations of the southern and western spiny forests are on average larger than on the rest of the island. Dry and spiny forest populations are reddish to orange in color, whereas rainforest populations are brown.

The allopatric population of Crematogaster dentata stat. nov. on Mauritius shows 8.8-11.2\% divergence with respect to Madagascar. The decision of whether or not to give species status to this population is arbitrary, since it is unknown if reproductive isolation exists with the mainland. A species name would already be available (with $C$. sewellii mauritiana). We decided against describing a separate species for Mauritius, since we were not able to find any distinct diagnostic morphological characters for this population.

Crematogaster lobata Emery, 1895

Fig. 12

Crematogaster sevellei var. lobata Emery, 1895: 342.

Crematogaster pacifica Santschi, 1919: 236. Worker holotype (by monotypy) from MADAGASCAR (Leg. J. de Gaulle) [NHMB, examined]. Lectotype worker by present designation: CASENT0101160, image on AntWeb. syn. nov.

Crematogaster sevellei lobata - Forel 1907: 80 (footnote).

Crematogaster sewellii lobata var. gigantea - Forel 1907: 80 (footnote) (w.) MADAGASCAR. Unavailable name (Bolton 1995: 153).

Crematogaster (Acrocoelia) sevellei var. lobata - Emery 1922: 148.

Crematogaster (Crematogaster) pacifica - Wheeler 1922b: 1023.

Crematogaster lobata - Santschi 1930: 63.

Crematogaster lobata pacifica - Santschi 1930: 63.

Crematogaster (Crematogaster) sevellei var. lobata - Bolton 1995: 166. 


\section{Diagnosis}

Crematogaster lobata is easily recognizable by its small eyes $(\mathrm{OI}<0.21)$ and lack of pronotal spines. These characters are otherwise only shared with $C$. mafybe sp. nov.; from the latter C. lobata can be distinguished by the absence of a distinct median longitudinal groove on the pronotum. In our molecular analysis, $C$. lobata is shown to have $14.3-19.9 \%$ mean sequence divergence in COI with regard to the rest of species in the $C$. degeeri-assemblage.

\section{Type material examined}

(MSNG): MADAGASCAR (Ch. Alluaud), 1 worker, lectotype worker by present designation [CASENT0102055; imaged on Antweb].

\section{Other material examined}

(BBBC, CASC, MCZC, MSNG, NHMB, PSWC): refer to supplementary Supplementary Material 1.

\section{Worker}

\section{Measurements}

$(\mathrm{n}=20)$ HW 0.80-1.38; HL 0.78-1.33; EL 0.15-0.23; SL 0.57-0.90; WL 0.86-1.42; SPL 0.00; PTH 0.15-0.24; PTL 0.26-0.38; PTW 0.25-0.46; PPL 0.15-0.25; PPW 0.24-0.39; LHT 0.63-1.00; CI 1.00-1.09; OI 0.16-0.21; SI 0.66-0.80; SPI 0.00; PTHI 0.50-0.68; PTWI 0.95-1.27; PPI 1.41-1.74; LBI 1.29-1.44.

\section{Description}

(Fig. 12A, C, D) Small to very large size (HW 0.80-1.38, WL 0.86-1.42); this species seems to have a polymorphic worker caste reminiscent of majors and minors.

Masticatory margin of mandibles with four teeth; head shape quadrate, mostly as long as wide (CI 1.001.09); posterior margin of head in full-face view laterally angular; occipital carinae usually distinct; antennal scapes short, not reaching head margin; midline of eyes situated slightly above midline of head in full-face view; eyes very small (OI 0.16-0.21), and confluent with lateral head margin in full-face view.

Pronotum laterally subangular; promesonotal suture usually indistinct in small workers and mesonotum more or less forming one plane with pronotum; in large workers, mesonotum often slightly raised with respect to pronotum and a median tubercule is present; mesonotum usually with a short, distinct posterior face; mesonotum angular laterally, ending in posterolateral tubercules; metanotal groove fairly shallow; propodeal spines absent, propodeum tuberculate directly above spiracles; dorsal face of propodeum short, posterior face of propodeum gently sloping; petiole in dorsal view broadly oval and moderately concave, without dorsolateral carinate margins and posterolateral tubercules or denticles; subpetiolar process variable, but often entirely absent; postpetiole wider than long, distinctly bilobed with a narrow median impression; subpostpetiolar process absent.

Head sculpture aciculate; promesonotum usually dorsally reticulate; propleuron aciculate, mesopleuron areolate, metapleuron reticulate-carinulate; dorsal face of propodeum carinulate, posterior face reticulate; dorsal face of petiole reticulate, helcium reticulate; postpetiole dorsally reticulate; lateral and ventral face of petiole and postpetiole reticulate; face usually with sparse (two to eight) erect, longer setae, and abundant, short, appressed to suberect pubescence; erect pilosity on promesonotum highly variable, often very abundant, usually including at least eight long setae; otherwise promesonotum dorsally with regular to sparse, appressed to subdecumbent pubescence; propodeum usually laterally with long, erect setae (> six); petiole and postpetiole usually with a pair of short erect setae posterolaterally, and shorter 
appressed pubescence; abdominal tergites four to seven with sparse, short, erect pilosity more abundant ventrally, and regular appressed to decumbent pubescence throughout. Color light brown to dark brown, with abdominal segments four to seven usually black.

\section{Queen and male}

Unknown.
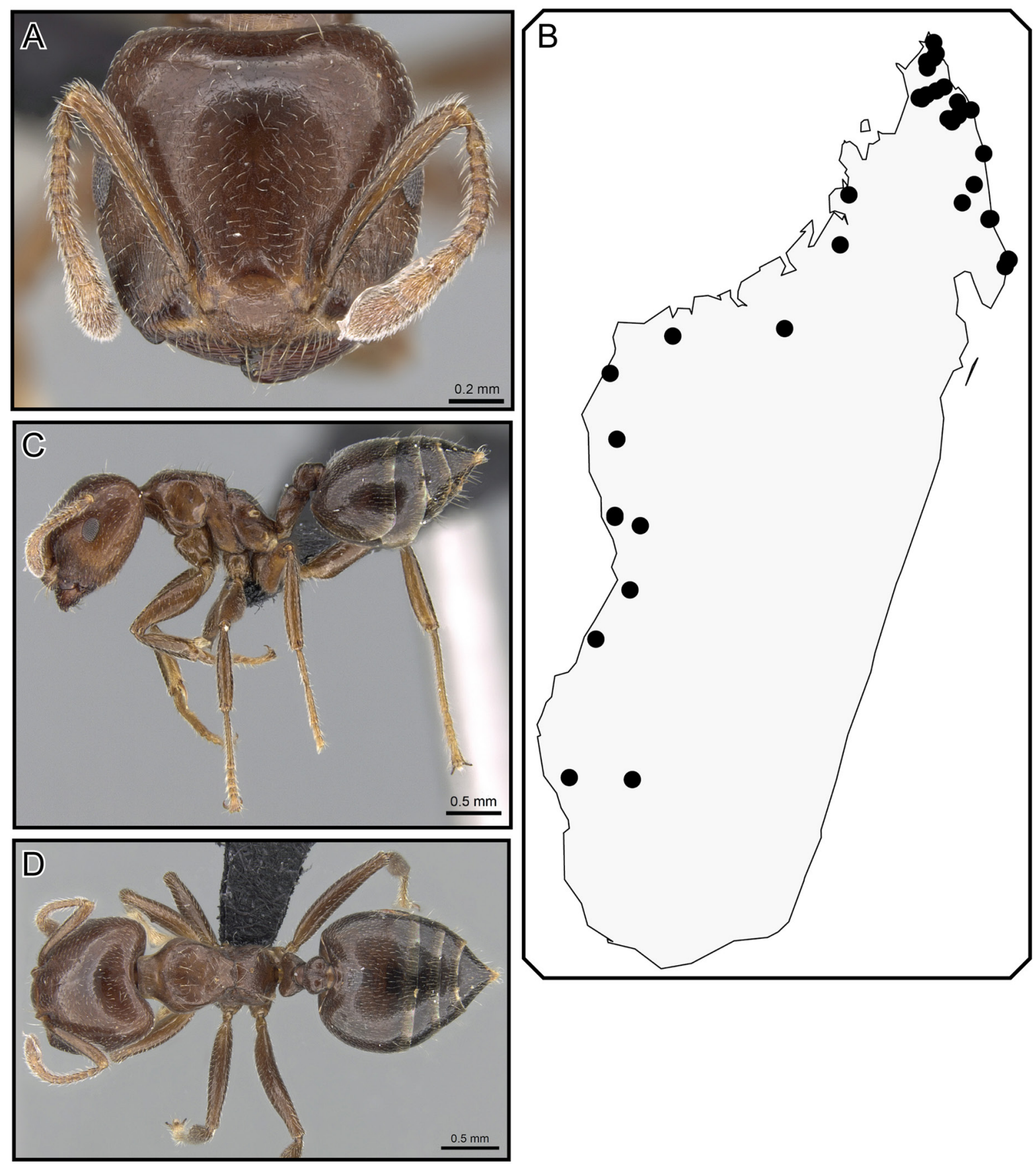

Fig. 12. Crematogaster lobata Emery, 1895, worker and distribution.A, C-D. Worker(CASENT0439060). A. Full-face. C. Profile. D. Dorsal. B. Species distribution. 


\section{Distribution and biology}

MADAGASCAR. Crematogaster lobata is found throughout the dry and littoral forests of western and northern Madagascar (Fig. 12B), mostly at low elevations ranging from $0 \mathrm{~m}$ to $400 \mathrm{~m}$. The species seems to occur in fairly low abundance. Some isolated records exist from rainforest and gallery forest up to $770 \mathrm{~m}$ altitude. Crematogaster lobata co-occurs often with $C$. dentata stat. nov., C. maina sp. nov., C. ramamy sp. nov. and C. tricolor stat. rev., as well as with $C$. sewellii at RS Beanka. The few colony collections available indicate that this species nests arboreally in dead branches and twigs. Crematogaster lobata is attracted to fish baits during both day and night baiting studies.

Crematogaster mafybe Blaimer sp. nov. urn:1sid:zoobank.org:act:66E18466-A2C6-4EA5-A92D-1D74D7A74CC5

Figs 13-14

\section{Diagnosis}

Crematogaster mafybe sp. nov. can be readily distinguished by its small eyes (OI 0.18-0.21) and lack of pronotal spines. These characters are otherwise only shared with C. lobata; from the latter C. mafybe sp. nov. can be distinguished by the presence of a distinct median longitudinal groove on the pronotum. Our data shows a mean sequence divergence between $C$. mafybe sp. nov. and the other species in the $C$. degeeri-assemblage of $12.7-21.3 \%$ (Table 3 ).

\section{Etymology}

The Malagasy word mafybe means "very strong", as this species is one of the largest and most robust Crematogaster species in Madagascar. The name is treated as a noun in apposition.

\section{Type specimens}

\section{Holotype}

Worker: pinned, CASENT0317762, BBB224, ex dead tree [imaged on AntWeb]; original locality label: M'dgascar: Fianarantsoa: P.N.Andringitra, $15.5 \mathrm{~km} \mathrm{~S}$ Ambalamanenjana, $1730 \mathrm{~m}, 22^{\circ} 12.453$ 'S, 46ํ58.314'E, 4 Mar. 2010, montane rainforest, B.B.Blaimer, BBB224; deposited at CASC.

\section{Paratypes}

4 workers, pinned, same collection and locality data as holotype. \#1: CASENT0317793, deposited at SAMC; \#2. CASENT0317794, deposited at MHNG. \#3: CASENT0317795, deposited at MCZC. \#4: CASENT0317796, deposited at UCDC.

\section{Type locality}

MADAGASCAR: Fianarantsoa: P.N. Andringitra: -22.2076, 46.9719, 1730 m, montane rainforest.

\section{Other material examined}

(BBBC, CASC, MCZC, PSWC): refer to Supplementary Material 1.

\section{Worker}

\section{Measurements}

$(\mathrm{n}=21)$ [holotype] HW 0.98-1.43 [1.24]; HL 0.89-1.30 [1.16]; EL 0.18-0.27 [0.21]; SL 0.63-0.94 [0.83]; WL 0.98-1.48 [1.26]; SPL 0.00-0.03 [0.02]; PTH 0.19-0.27 [0.22]; PTL 0.27-0.44 [0.35]; PTW 0.340.52 [0.45]; PPL 0.16-0.25 [0.23]; PPW 0.26-0.40 [0.33]; LHT 0.76-1.23 [0.98]; CI 1.04-1.11 [1.07]; OI 0.18-0.21 [0.18]; SI 0.60-0.79 [0.72]; SPI 0.00-0.03 [0.01]; PTHI 0.59-0.84 [0.62]; PTWI 1.06-1.41 [1.27]; PPI 1.39-1.79 [1.45]; LBI 1.15-1.42 [1.29]. 


\section{Description}

(Fig.13A-C) Medium to very large size (HW 0.98-1.43, WL 0.98-1.48).

Masticatory margin of mandibles with four teeth; head shape quadrate, more or less as long as wide (CI 1.04-1.11); posterior margin of head in full-face view laterally angular or subangular, sometimes medially depressed; occipital carinae usually distinct; antennal scapes of variable length, not reaching or surpassing head margin; midline of eyes situated at midline of head in full-face view; eyes small (OI $0.18-0.21$ ), and confluent with lateral head margin in full-face view.
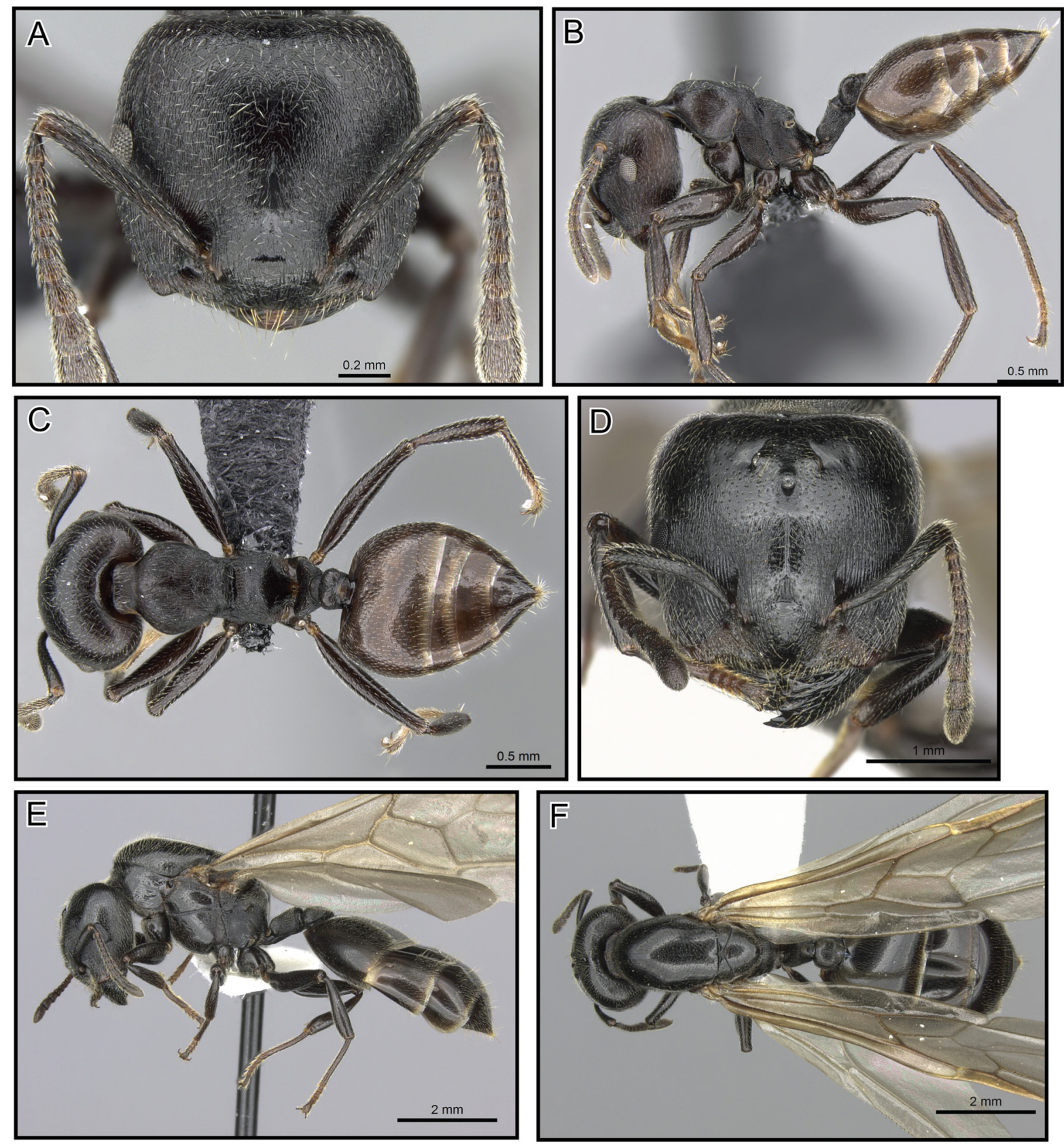

Fig. 13. Crematogaster mafybe sp. nov., worker and queen. A-C. Worker (CASENT0317762). A. Fullface. B. Profile. C. Dorsal. D-F. Queen (CASENT0193464). D. Full-face. E. Profile F. Dorsal. 
Pronotum laterally angular; promesonotal suture usually indistinct, the mesonotum more or less forming one plane with pronotum; pronotum with a longitudinal groove; mesonotum usually without distinct posterior face; mesonotum laterally angular, ending in small posterolateral tubercules; metanotal groove fairly shallow; propodeal spines absent, or propodeum with small denticules or tubercules; dorsal face of propodeum very short to absent, posterior face of propodeum sloping fairly steeply, often with sharp transverse groove; petiole in dorsal view broadly oval to moderately flared, concave, without dorsolateral carinate margins and posterolateral tubercules or denticles; subpetiolar process variable, but often entirely absent; postpetiole bilobed with a narrow to broad median impression; subpostpetiolar process absent.

Head sculpture carinulate to reticulate; promesonotum carinulate to reticulate; propleuron reticulate, mesopleuron areolate, metapleuron reticulate-carinulate; dorsal face of propodeum carinulate to reticulate, posterior face reticulate; petiole and postpetiole dorsally and ventrally reticulate; erect pilosity on face sparse, < eight longer setae, and abundant, short, appressed to suberect pubescence; erect pilosity on promesonotum variable, usually $<12$ long setae; otherwise promesonotum dorsally with very sparse appressed to subdecumbent pubescence; petiole and postpetiole usually with a pair of short erect setae posterolaterally, and shorter appressed pubescence; abdominal tergites four to seven with very sparse short erect pilosity, ventrally more abundant, and regular appressed to decumbent pubescence throughout. Color black or brown, abdominal segments four to seven yellowish.

\section{Queen}

\section{Measurements}

$(\mathrm{n}=5)$ HW 1.70-2.18; HL 1.58-2.06; EL 0.40-0.48; SL 0.93-1.18; MSNW 1.35-1.54; MSNL 1.40-1.95; WL 2.56-3.34; SPL 0.00; PTH 0.38-0.53; PTL 0.51-0.65; PTW 0.64-0.79; PPL 0.38-0.50; PPW 0.560.71; LHT 1.08-1.42; CI 0.99-1.08; OI 0.23-0.26; SI 0.57-0.59; MSNI 0.79-1.02; SPI 0.00; PTHI 0.730.90; PTWI 1.21-1.26; PPI 1.28-1.74; LBI 2.22-2.36.

\section{Description}

(Fig. 13D-F) Large to very large size (HW 1.70-2.18, WL 2.56-3.34). With worker characters, except as follows.

Masticatory margin of mandibles with five teeth; antennal scapes short, usually not surpassing level of lateral ocelli; eyes small (OI 0.23-0.26), situated at or slightly below midline of head in full-face view; head shape more or less quadrate (CI 0.99-1.08), posterior margin straight.

Mesoscutum in dorsal view usually longer than wide (MSNI 0.79-1.02); dorsal face of propodeum short, meeting posterior face at a right angle; propodeal spines or tubercules absent; petiole broadly flared, postpetiole merely impressed posteriorly; subpetiolar process absent.

Head sculpture aciculate to carinulate; otherwise aciculate to reticulate throughout; face usually with abundant, longer, erect setae and dense, short, erect to decumbent pubescence; mesonotum with dense, short, suberect pubescence, and interspersed longer, erect setae; petiole and postpetiole with dense short suberect to decumbent pilosity, and postpetiole with additional longer dorso-posterior setae; abdominal tergites and sternites four to seven with abundant erect pilosity and dense appressed pubescence. Colored black; wings with a brown hue.

\section{Male}

\section{Measurements}

$(\mathrm{n}=3)$ HW 0.84-0.88; HL 0.68-0.71; EL 0.27-0.31; SL 0.17-0.18; MSNW 1.07-1.13; MSNL 0.96-1.09; WL 1.82-2.10; SPL 0.00; PTH 0.24-0.30; PTL 0.26-0.36; PTW 0.33-0.41; PPL 0.18-0.22; PPW 0.37- 
0.43; LHT 0.97-1.01; CI 1.22-1.24; OI 0.40-0.44; SI 0.25-0.27; MSNI 1.03-1.12; SPI 0.00; PTHI 0.820.94; PTWI 1.13-1.26; PPI 1.91-2.09; LBI 1.84-2.07.

\section{Description}

(Fig. 14A, C) Large males (HW 0.84-0.88, WL 1.82-2.10).

Mandibles short and reduced, one minute apical tooth present; clypeus not much protruding; eyes fairly small (OI 0.40-0.44); midline of eyes situated well below midline of head, almost extending to clypeal margin; antennae 12-segmented; head wider than long (CI 1.22-1.24); in full-face view ocellar triangle situated below posterior head margin, elevated with respect to rest of face; occipital carinae very distinct, with a thin flange projecting backwards.

Mesoscutum in dorsal view as wide as or slightly wider than long (MSNI 1.03-1.12); scutellum in dorsal view broadly tapering from anterior to posterior end, dorsoposterior part rounded; metanotum protruding only slightly from below scutellum; dorsal face of propodeum short; propodeal spines absent; petiole in dorsal view oval, laterally rounded, in lateral view anteriorly greatly tapering; subpetiolar process absent; postpetiole fairly flat and short, lacking median impression.

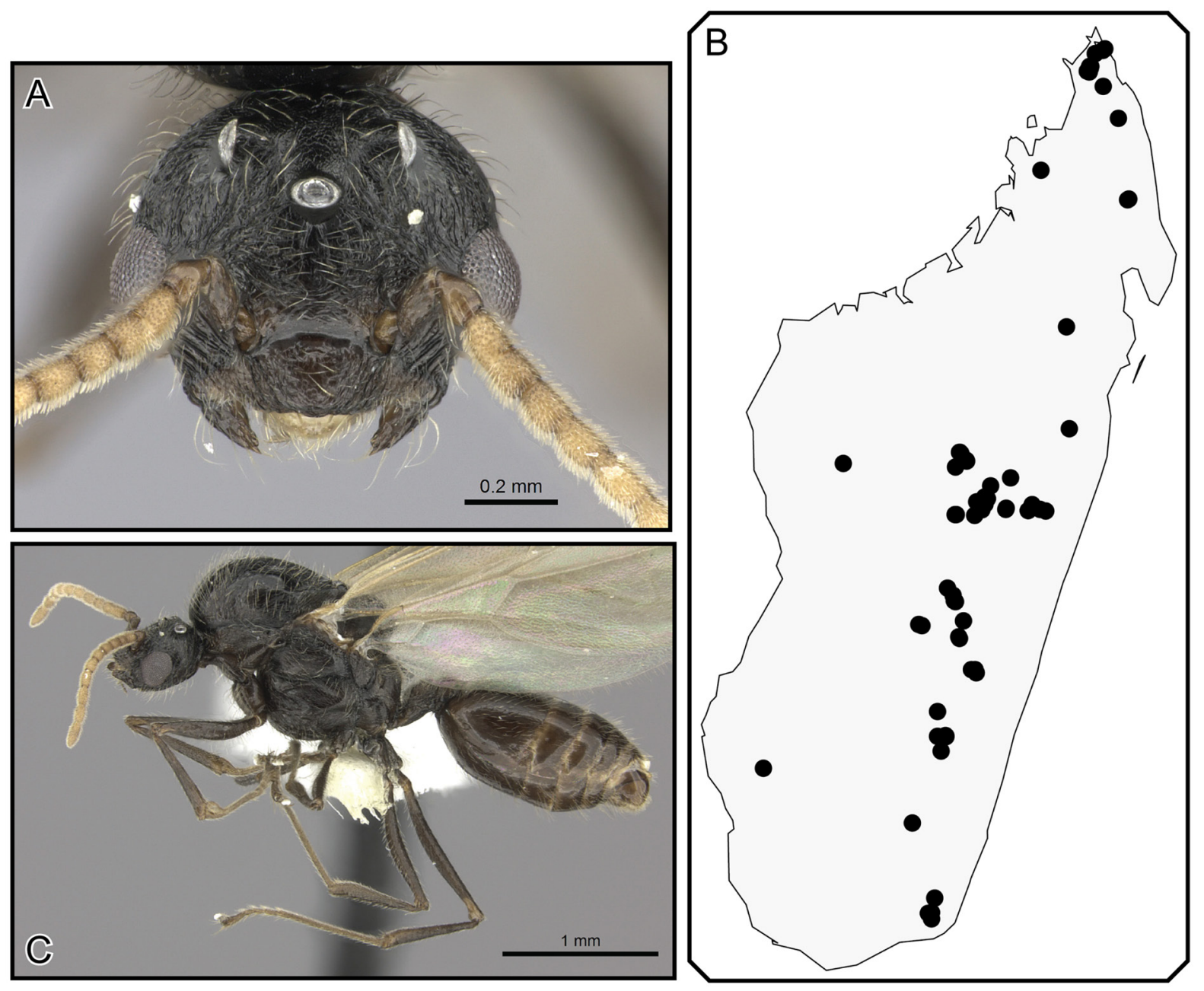

Fig. 14. Crematogaster mafybe sp. nov., male and distribution. A, C. Male (CASENT0193465). A. Fullface. C. Profile. B. Species distribution. 
Head sculpture rugulose-reticulate; mesosoma dorsally with very fine longitudinal carinulae; otherwise sculpture more or less reticulate to aciculate; dense, short, erect to decumbent pubescence covering head, meso- and metasoma, interspersed with a few longer, erect setae. Color black.

\section{Distribution and biology}

MADAGASCAR. Crematogaster mafybe sp. nov. is a high-elevation specialist mostly found throughout the rainforest and montane forests of the central and south-central highland region and northern Madagascar from $700 \mathrm{~m}$ to $2150 \mathrm{~m}$ in elevation (Fig. 14B). Interesting disjunct populations exist in western Madagascar in the isolated montane forests of Analavelona and RS Ambohijanahary. There are some records of this species from coastal littoral and dry forest in northern Madagascar (see Supplementary Material 1) at low elevations, but we suspect that this habitat is suboptimal for this species. Crematogaster mafybe sp. nov. co-occurs mostly with $C$. degeeri, $C$. alafara sp. nov. and $C$. malahelo sp. nov. in montane habitats, but is also found in sympatry with $C$. tricolor stat. rev., $C$. dentata stat. nov., C. maina sp. nov., C. ramamy sp. nov., C. lobata and $C$. sewellii at several locations. This species has been collected mostly from nests in dead branches and twigs above ground, and we thus assume that its preferred nesting sites are arboreal. In montane habitats, however, this species also nests in rotten logs on the ground and under stones.

Crematogaster maina Blaimer sp. nov. urn:1sid:zoobank.org:act:A59911D0-2601-405F-91CF-355998892F4E

Figs 15-16

\section{Diagnosis}

Crematogaster maina sp. nov. is easily distinguished from other species in the $C$. degeeri-assemblage by its yellow body coloration. It is considerably smaller than C. bara sp. nov., which shares its yellow color (HW 0.60-0.86, WL 0.66-0.93 vs C. bara sp. nov. HW 0.87-1.03, WL 0.98-1.22) and shorter antennal scapes (SI 0.71-0.83 vs C. bara sp. nov. SI 0.91-1.01). Molecular sequence divergence to the remaining species ranges from $11.3-20.2 \%$.

\section{Etymology}

This species is named after its predominant distribution in the dry forests of Madagascar: maina is the Malagasy word for "dry." The name is treated as a noun in apposition.

\section{Type specimens}

\section{Holotype}

Worker: pinned, CASENT0317758, BBB246, ex dead twig above ground [imaged on AntWeb]; original locality label: M'dgascar: Toliara: PN Andohahela, p3: Ankasofotsy, $4.5 \mathrm{~km}$ W Ranopiso, S 25 00.820 ', E 4638.790', 160 m, 21 Mar. 2010, transition forest, B.B. Blaimer, BBB\#246; deposited at CASC.

\section{Paratypes}

4 workers, pinned, same collection and locality data as holotype. \#1: CASENT0317789, deposited at SAMC. \#2: CASENT0317790, deposited at MHNG. \#3: CASENT0317791, deposited at MCZC. \#4: CASENT0317792, deposited at UCDC.

\section{Type locality}

MADAGASCAR: Toliara: P.N. Andohahela (parcel 3): -25.0137, 46.6465, $160 \mathrm{~m}$, dry forest/transition forest. 


\section{Other material examined}

(BBBC, CASC, MCZC, PSWC): refer to Supplementary Material 1.

\section{Worker}

\section{Measurements}

$(\mathrm{n}=21)$ [holotype] HW 0.60-0.86 [0.77]; HL 0.56-0.80 [0.74]; EL 0.13-0.20 [0.18]; SL 0.43-0.64 [0.59]; WL 0.66-0.93 [0.87]; SPL 0.05-0.12 [0.10]; PTH 0.12-0.21 [0.18]; PTL 0.19-0.27 [0.26]; PTW 0.260.36 [0.30]; PPL 0.10-0.19 [0.15]; PPW 0.21-0.34 [0.28]; LHT 0.44-0.64 [0.60]; CI 1.02-1.12 [1.04]; OI 0.20-0.26 [0.24]; SI 0.71-0.83 [0.80]; SPI 0.07-0.14 [0.11]; PTHI 0.63-0.81 [0.69]; PTWI 1.13-1.44 [1.13]; PPI 1.61-2.02 [1.84]; LBI 1.10-1.69 [1.45].

\section{Description}

(Fig. 15A-F) Very small to small size (HW 0.60-0.86, WL 0.66-0.93).

Masticatory margin of mandibles with four teeth; head shape fairly quadrate or slightly wider than long (CI 1.02-1.12); posterior margin of head in full-face view laterally subangular or rounded, sometimes slightly medially depressed; occipital carinae distinct; antennal scapes usually about reaching head margin; midline of eyes situated at or slightly above midline of head in full-face view; eyes moderately large (OI 0.20-0.26) and only slightly protruding from head margin.

Pronotum laterally subangular, often carinate; usually promesonotal suture indistinct, and mesonotum forming a flat plane with respect to pronotum; in larger workers (Fig. 15D-F) promesonotal suture present, and mesonotum raised and anteriorly projecting over pronotum; mesonotum usually without a posterior face; mesonotum posterolaterally with raised carinae or sometimes small denticles, but not distinctly set off from propodeum, forming one convex outline with the latter in lateral view; metanotal groove very shallow, laterally bordered by carinae but not constricted; propodeal spines short (SPI 0.07-0.14), straight and spiniform; dorsal face of propodeum very short or absent; posterior face of propodeum usually gently sloping; petiole in dorsal view broadly or sharply flared and convex, dorsolaterally rounded, without posterolateral tubercules; subpetiolar process mostly present as sharp acute tooth; postpetiole very distinctly bilobed, separated by a broad median impression; subpostpetiolar process absent.

Head sculpture aciculate; pronotum costulate, carinulate or reticulate, propodeum often carinulate; rest of mesosoma, petiole, postpetiole reticulate; face usually with $<$ ten erect longer setae, and regular shorter appressed pubescence; erect pilosity on promesonotum usually restricted to lateral margins, usually $<$ ten setae; otherwise promesonotum dorsally with sparse appressed to subdecumbent pubescence; petiole and postpetiole usually with a pair of short erect setae posterolaterally, and shorter appressed pubescence; abdominal tergites and sternites four to seven with abundant, short, erect pilosity, and sparse appressed to decumbent pubescence throughout. Color uniformly yellow.

\section{Queen}

\section{Measurements}

(n = 5) HW 0.98-1.13; HL 1.04-1.17; EL 0.29-0.34; SL 0.66-0.73; MSNW 0.86-1.02; MSNL 0.96-1.05; WL 1.65-1.82; SPL 0.00; PTH 0.31-0.34; PTL 0.36-0.41; PTW 0.45-0.50; PPL 0.22-0.30; PPW 0.400.47; LHT 0.72-0.87; CI 0.94-1.00; OI 0.27-0.31; SI 0.61-0.66; MSNI 0.85-0.99; SPI 0.00; PTHI 0.830.90; PTWI 1.18-1.28; PPI 1.50-1.79; LBI 2.14-2.38.

\section{Description}

(Fig. 16A-C) Very small to small size (HW 0.98-1.13, WL 1.65-1.82). With worker characters, except as follows. 
Masticatory margin of mandibles with five teeth; antennal scapes usually not surpassing level of lateral ocelli; eyes moderately large (OI 0.27-0.31), situated slightly below midline of head in full-face view; head shape square or rectangular-elongate (CI 0.94-1.00); posterior margin straight.

Mesoscutum in dorsal view usually longer than wide (MSNI 0.85-0.99); dorsal face of propodeum short; propodeal spines or tubercules absent; petiole moderately to greatly flared, postpetiole with a complete, but superficial impression; subpetiolar process variable, usually absent.

Sculpture aciculate throughout; face usually with $<$ ten longer erect setae and abundant, shorter, erect to suberect pilosity; mesonotum with abundant short, and scattered longer, erect setae; petiole and postpetiole with dense short suberect to decumbent pilosity, and postpetiole with additional longer dorso-posterior setae; abdominal tergites and sternites four to seven with sparse, scattered erect pilosity and regular appressed pubescence. Color as in workers; wings clear.
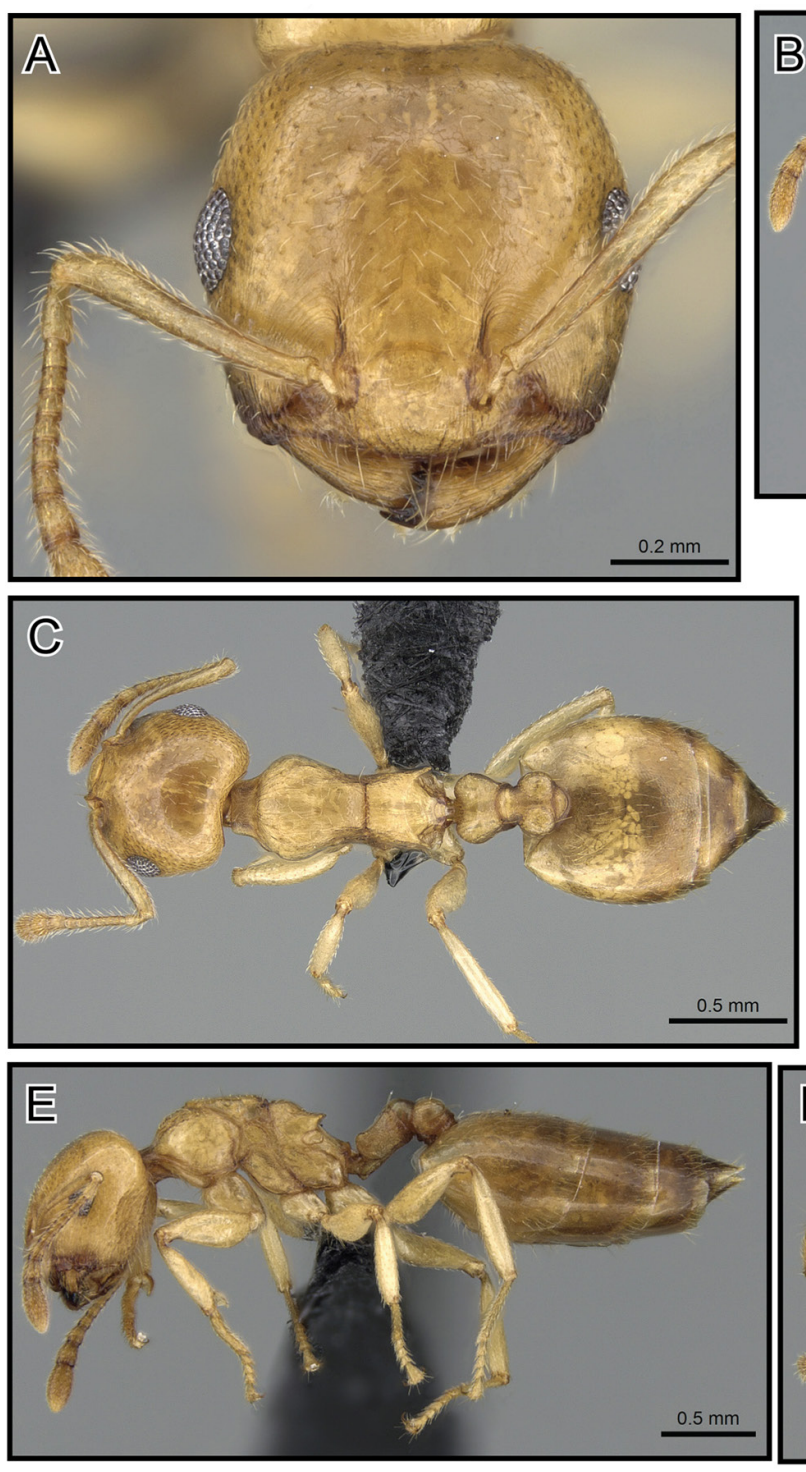

Fig. 15. Crematogaster maina sp. nov., workers. A-C. Worker (CASENT0317758). A. Full-face. B. Profile. C. Dorsal. D-F. Worker (CASENT0317760). D. Full-face. E. Profile. F. Dorsal.
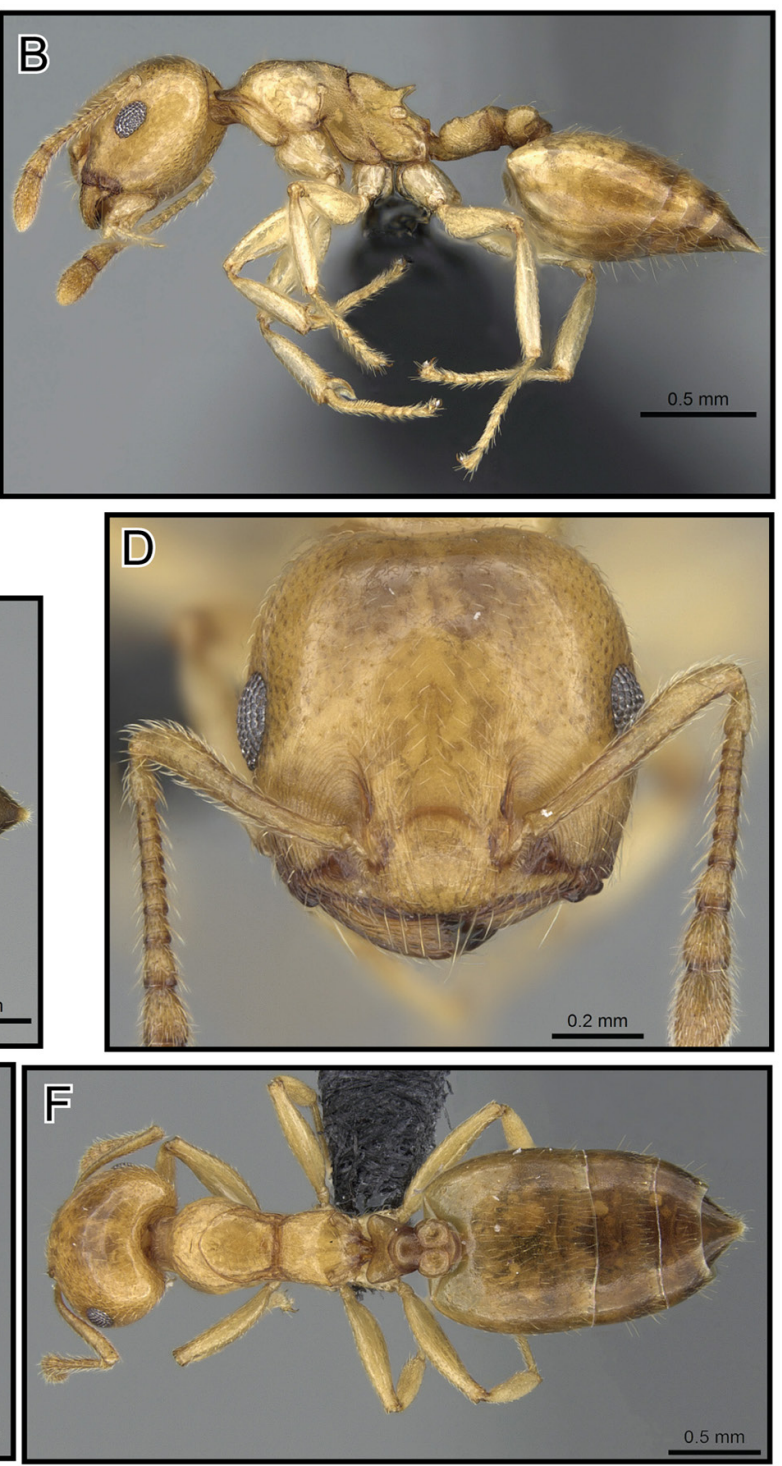

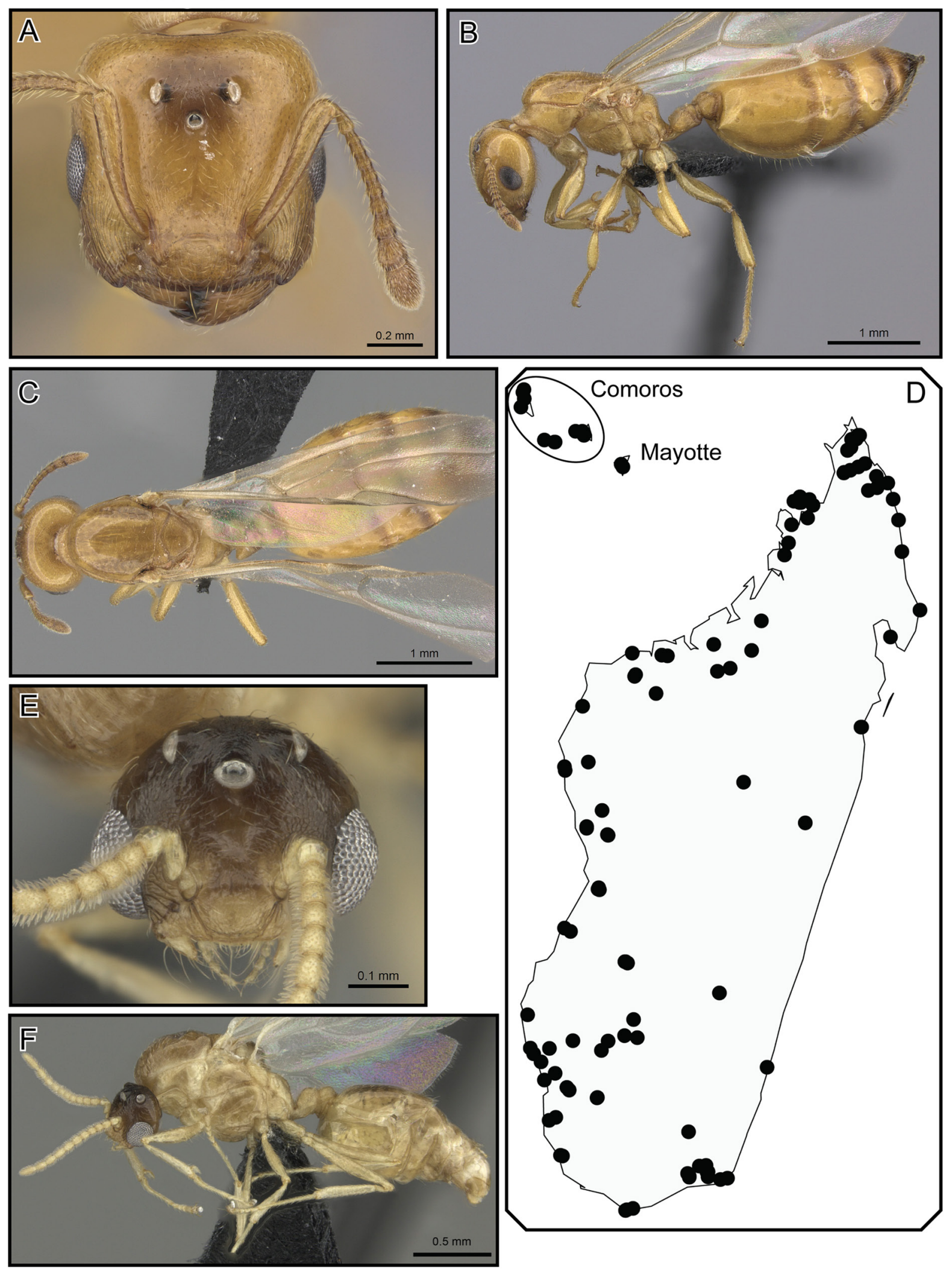

Fig. 16. Crematogaster maina sp. nov., queen, male and distribution. A-C. Queen (CASENT0485040). A. Full-face. B. Profile. C. Dorsal. D. Species distribution. E-F. Male (CASENT0455252). E. Full-face. F. Profile. 
Male

\section{Measurements}

$(\mathrm{n}=3)$ HW 0.57-0.59; HL 0.45-0.48; EL 0.22-0.23; SL 0.10-0.11; MSNW 0.67-0.74; MSNL 0.61-0.66; WL 1.19-1.24; SPL 0.00; PTH 0.17-0.20; PTL 0.22-0.24; PTW 0.22-0.25; PPL 0.12-0.15; PPW 0.250.27; LHT 0.60-0.64; CI 1.23-1.31; OI 0.48; SI 0.20-0.25; MSNI 1.02-1.18; SPI 0.00; PTHI 0.81-0.84; PTWI 0.93-1.17; PPI 1.74-2.02; LBI 1.94-2.03.

\section{Description}

(Fig. 16E-F) Very small (HW 0.57-0.59, WL 1.19-1.24).

Mandibles very short and reduced, with one singular apical tooth; clypeus not much protruding; eyes fairly large (OI 0.48) and protruding, midline of eyes situated well below midline of head, almost extending to clypeal margin; antennae 12-segmented; head wider than long (CI 1.23-1.31); in full-face view ocellar triangle situated below posterior head margin, elevated with respect to rest of face; occipital carinae distinct.

Mesoscutum in dorsal view as wide as to a little wider than long (MSNI 1.02-1.18); scutellum in dorsal view broadly tapering from anterior to posterior end, dorsoposterior part rounded; metanotum protruding from below scutellum; dorsal face of propodeum short; propodeal spines absent; petiole in dorsal view oval, laterally rounded, in lateral view anteriorly greatly tapering; subpetiolar process absent; postpetiole fairly flat and short, lacking median impression.

Head sculpture reticulate; mesoscutum with very fine longitudinal carinulae; otherwise sculpture more or less shiny to aciculate; sparse appressed to decumbent pubescence on head and mesosoma, a few short erect setae on face may be present; rest of pilosity similar to queens. Color pale to light brown.

\section{Distribution and biology}

MADAGASCAR, COMOROS, MAYOTTE. Crematogaster maina sp. nov. is a very abundant species at low elevations throughout the dry forests, littoral forests and spiny forests of Madagascar, the Comoros Islands and Mayotte (Fig. 16D). It has also been collected at higher elevations in more humid habitats (up to $1410 \mathrm{~m}$ ), but we suspect that some of these occurrences could represent tramp-like introductions to suboptimal habitats. Crematogaster maina sp. nov. is commonly found in sympatry with $C$. dentata stat. nov., C. ramamy sp. nov., C. tricolor stat. rev. and C. lobata, and at some locations is also known to co-occur with $C$. mafybe sp. nov., C. sewellii, $C$. bara sp. nov. and C. masokely sp. nov. The species is most often encountered nesting arboreally in dead twigs or branches. This species has only been found at baits during the night and has never been observed foraging during the day. We conclude that it is nocturnal, which would explain its pale coloration.

\section{Comments}

As discussed above, the two individuals of C. maina sp. nov. sampled for the genetic analysis are highly divergent from all but one of the rest of the Madagascar samples (13.3\% maximal distance; Table 3), and further do not group as closest relatives to these in our analysis (Figs 1 and 2A). However, since we cannot find conclusive morphological evidence to distinguish the island population from the one on Madagascar, we refrain from splitting the two. With only three individuals sampled, spurious sequences (discussed below) are a possibility. 
Crematogaster malahelo Blaimer sp. nov.

urn:1sid:zoobank.org:act:E66A64F7-7DBF-4580-8217-9E31A16A50D6

Fig. 17

\section{Diagnosis}

Workers of Crematogaster malahelo sp. nov. can usually be distinguished from other species in the $C$. degeeri-assemblage given the characteristic promesonotal structure with a propodeum usually distinctly set off from the promesonotum in lateral view (as in Fig. 17C). C. alafara sp. nov. could cause possible confusion with this species, but $C$. malahelo sp. nov. bears sharp lateral margins on the pronotum (absent in C. alafara sp. nov.) and has shorter, triangular spines (SPI 0.03-0.07).

Mean genetic distance for $C$. malahelo sp. nov. to other species within the $C$. degeeri-assemblage in the barcode region is $10.9-19.6 \%$ (Table 3 ).

\section{Etymology}

The Malagasy word malahelo means "sad." This name alludes to the rather sad fact that all three of the montane rainforest locations where this species is known to occur is heavily disturbed. The name is treated as noun in apposition.

\section{Type specimens}

\section{Holotype}

Worker: pinned, CASENT0317768, BLF17431, ex dead twig above ground [imaged on AntWeb]; original locality label: MADG'R: Kaloy, 1423 m, 18 35.39'S, 47³9.06'E, 27 Apr. 2007, disturbed montane rainforest, Fisher et al., BLF\#; deposited at CASC.

\section{Paratypes}

4 workers, pinned. \#1: CASENT0317767, deposited at SAMC. \#2: CASENT0317769, deposited at MHNG. \#3: CASENT0317770, deposited at MCZC. \#4: CASENT0317771, deposited at UCDC. \#1 and \# 2 same collection and locality data as holotype. \#3 and \#4: BBB004, on tree trunk, Station Forestière Manjakatompo, -19.37083, 47.33900, 1575 m, disturbed montane rainforest, 31 Mar. 2007, B.B.Blaimer.

\section{Type locality}

MADAGASCAR: Antananarivo: Kaloy: -18.58998, 47.65102, 1423 m, disturbed montane rainforest.

\section{Other material examined}

(BBBC, CASC): refer to Supplementary Material 1.

\section{Worker}

\section{Measurements}

$(\mathrm{n}=10)$ [holotype] HW 0.86-1.03 [0.93]; HL 0.80-0.94 [0.80]; EL 0.18-0.24 [0.20]; SL 0.63-0.71 [0.63]; WL 0.92-1.08 [0.94]; SPL 0.03-0.07 [0.05]; PTH 0.19-0.25 [0.21]; PTL 0.25-0.31 [0.29]; PTW 0.330.43 [0.43]; PPL 0.15-0.20 [0.17]; PPW 0.28-0.37 [0.34]; LHT 0.64-0.77 [0.67]; CI 1.03-1.16 [1.16]; OI 0.22-0.26 [0.25]; SI 0.74-0.80 [0.79]; SPI 0.03-0.07[0.06]; PTHI 0.66-0.82 [0.72]; PTWI 1.24-1.52 [1.52]; PPI 1.71-2.04 [2.04]; LBI 1.36-1.53 [1.40].

\section{Description}

(Fig. 17A, C-D) Small to medium size (HW 0.86-1.03, WL 0.92-1.08).

Masticatory margin of mandibles with four teeth; head shape fairly quadrate or slightly wider than long (CI 1.03-1.16); posterior margin of head in full-face view laterally subangular or rounded, sometimes 
slightly medially depressed; occipital carinae distinct; antennal scapes usually about reaching or surpassing head margin; midline of eyes situated at or slightly above midline of head in full-face view; eyes moderately large (OI 0.22-0.26), slightly protruding.

Pronotum laterally angular, with sharp, raised margins; promesonotal suture indistinct, mesonotum more or less forming one plane with pronotum, but often the two separated by distinct variation in sculpture (see below); mesonotum usually without a distinct posterior face; laterally mesonotum angular to carinate, often with posterolateral denticles setting off the promesonotum distinctly from propodeum; metanotal groove fairly shallow, laterally constricted; propodeal spines very short (SPI 0.03-0.07),

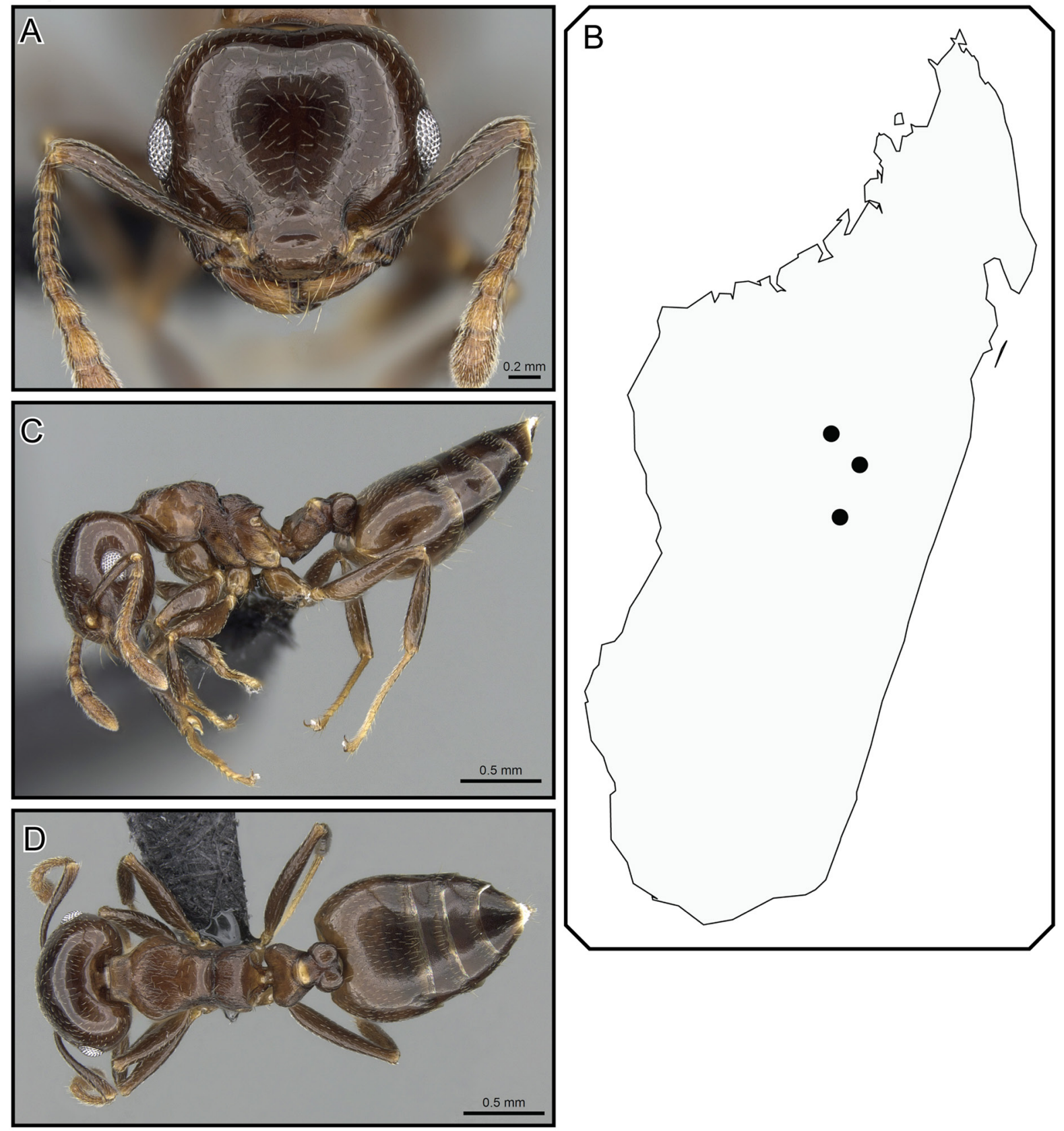

Fig. 17. Crematogaster malahelo sp. nov., worker and distribution. A, C-D. Worker (CASENT0317768). A. Full-face. C. Profile. D. Dorsal. B. Species distribution. 
triangular dents or spines; dorsal face of propodeum distinct, convex; posterior face of propodeum steeply sloping; petiole in dorsal view from broadly to strongly flared and usually strongly concave, dorsolaterally carinate but without posterolateral tubercules or denticles; subpetiolar process usually present as small rounded tooth; postpetiole wider than long, very distinctly bilobed, with broad median impression; subpostpetiolar process absent.

Head sculpture shiny to aciculate; pronotum dorsally costulate and contrasting abruptly with a shiny to aciculate mesonotum; dorsal face of propodeum carinulate or reticulate; rest of mesosoma, petiole and postpetiole aciculate to reticulate; face usually with $<$ four erect, longer setae, and regular, shorter, appressed to suberect pubescence; promesonotum usually with < eight erect, long setae; otherwise promesonotum dorsally with sparse appressed to subdecumbent pubescence; petiole and postpetiole usually with a pair of short, erect setae posterolaterally, and shorter suberect, decumbent or appressed pubescence; abdominal tergites four to seven with sparse, short, erect pilosity, more abundant and longer on sternites four to seven, and regular appressed to decumbent pubescence throughout. Color reddish brown to dark brown.

\section{Queen and male}

Queens and males remain unknown for this species.

\section{Distribution and biology}

MADAGASCAR. Crematogaster malahelo sp. nov. is a rare species endemic to montane forest of the central highlands in Madagascar (Fig. 17B). It has been collected from only three locations, all of which harbor unprotected, rather disturbed forest fragments (SF Manjakatompo, Andranorovitra, Kaloy). Given this restricted distribution the species is exclusively sympatric with C. mafybe sp. nov. and allopatric with the rest of the $C$. degeeri-assemblage. The few collections have been made from dead twigs above the ground, indicating arboreal nesting preferences.

\section{Crematogaster masokely Blaimer sp. nov. urn:1sid:zoobank.org:act:44FEB42B-C008-4831-BA5B-47AFF14CC7D9}

\section{Fig. 18}

\section{Diagnosis}

Crematogaster masokely sp. nov. can be distinguished from the rest of the $C$. degeeri-assemblage by a combination of the following characters: small eyes (OI 0.17-0.20), short antennal scapes (SI 0.67-0.75) and a tuberculate or denticulate mesonotum.

\section{Etymology}

Crematogaster masokely sp. nov. is named after the species' small eyes: maso is Malagasy for "eye" and kely means "small." The name is treated as a noun in apposition.

\section{Type specimens}

\section{Holotype}

Worker: pinned, CASENT0068954, BLF12840(9), ex sifted litter [imaged on Antweb]; original locality label: MADG'R: Toamasina: Ambohidena, 20 m, 24 Nov. 2005, 16²49.46'S, 4957.85'E, littoral forest, Fisher et al., BLF12840; deposited at CASC.

\section{Paratypes}

Workers not designated, due to the small number of existing specimens. 


\section{Type locality}

MADAGASCAR: Toamasina: Ile St. Marie, Forêt Ambohidena: -16.82433, 49.96417, 20 m, littoral forest.

\section{Other material examined}

(CASC, PSWC): refer to Supplementary Material 1.

\section{Worker}

\section{Measurements}

$(\mathrm{n}=11)$ [holotype] HW 0.72-0.91 [0.85]; HL 0.66-0.91 [0.83]; EL 0.13-0.18 [014]; SL 0.48-0.62 [0.60]; WL 0.74-0.95 [0.88]; SPL 0.01-0.06 [0.03]; PTH 0.15-0.19 [0.15]; PTL 0.21-0.29 [0.27]; PTW 0.23-
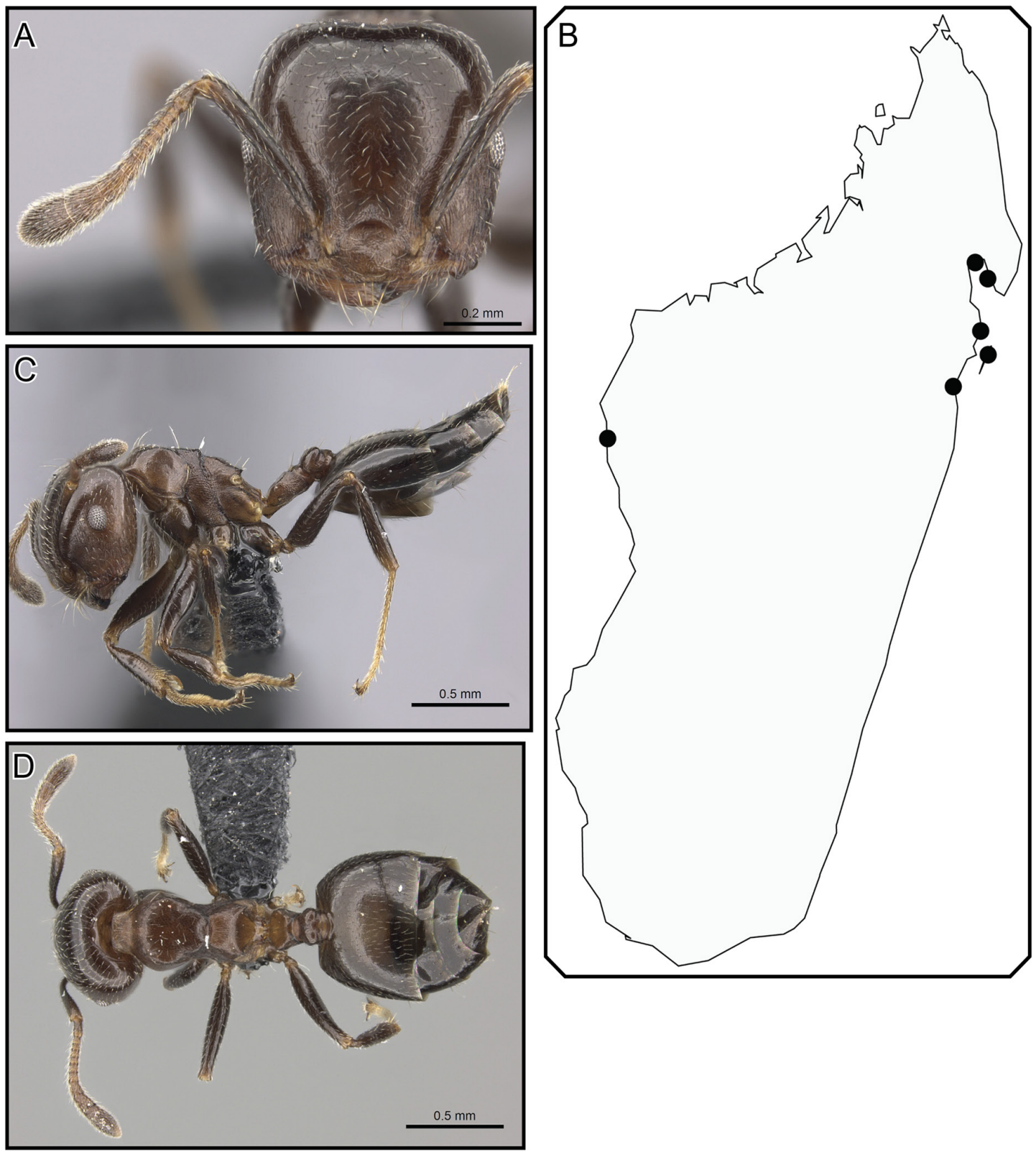

Fig. 18. Crematogaster masokely sp.nov, worker and distribution. A, C-D. Worker (CASENT0068954). A. Full-face. C. Profile. D. Dorsal. B. Species distribution. 
0.34[0.27]; PPL 0.12-0.19 [0.15]; PPW 0.19-0.28[0.24]; LHT 0.49-0.67 [0.63]; CI 0.99-1.09 [1.02]; OI 0.17-0.20 [0.17]; SI 0.67-0.75 [0.72]; SPI 0.01-0.06 [0.04]; PTHI 0.57-0.74 [0.57]; PTWI 1.01-1.23 [1.01]; PPI 1.39-1.75 [1.59]; LBI 1.33-1.68 [1.39].

\section{Description}

(Fig. 18A, C-D) Small (HW 0.72-0.91, WL 0.74-0.95).

Masticatory margin of mandibles with four teeth; head shape more or less quadrate (CI 0.99-1.09); posterior margin of head in full-face view laterally angular or subangular, sometimes medially depressed; occipital carinae usually distinct; antennal scapes usually short, not reaching or surpassing head margin; midline of eyes situated at midline of head in full-face view; eyes small (OI 0.17-0.20), and confluent with lateral head margin in full-face view.

Pronotum laterally angular; promesonotal suture usually indistinct, mesonotum more or less forming one plane with pronotum; mesonotum usually without distinct posterior face; laterally mesonotum angular, ending in small posterolateral denticles or tubercules, that set mesonotum off from propodeum; metanotal groove fairly shallow, laterally constricted; propodeum with small denticles or tubercules (SPI 0.01-0.06); dorsal face of propodeum short to indistinct, posterior face of propodeum gently sloping; petiole in dorsal view broadly oval, concave, without dorsolateral carinate margins and posterolateral tubercules or denticles; subpetiolar process variable, usually small angular dent; postpetiole bilobed with a narrow median impression; subpostpetiolar process absent.

Head sculpture aciculate; promesonotum reticulate to aciculate; mesopleuron areolate, dorsal face of propodeum carinulate to reticulate; otherwise sculpture reticulate to aciculate; erect pilosity on face sparse, usually $<$ four longer setae, and regular, short, appressed to suberect pubescence; promesonotum usually with $<$ ten long erect setae, and very sparse, appressed to subdecumbent pubescence; petiole and postpetiole usually with a pair of short erect setae posterolaterally, and sparse appressed pubescence; abdominal tergites four to seven with scattered short erect pilosity, ventrally more abundant, and regular appressed to decumbent pubescence throughout. Color medium to dark brown.

\section{Queen and male}

Queens and males of this species are unknown.

\section{Distribution and biology}

MADAGASCAR. Crematogaster masokely sp. nov. is highly endemic to the littoral forests of eastern Madagascar, with the exception of one peculiar disjunct distribution in the tsingy of the SF Beanka on the west coast (Fig. 18B). The species co-occurs with C. tricolor stat. rev., C. maina sp. nov., C. dentata stat. nov. and C. ramamy sp. nov. No colony collections of this species have been made, therefore nothing is known of its biology.

\section{Crematogaster ramamy Blaimer sp. nov. urn:Isid:zoobank.org:act:5CE5408D-724C-4F67-819C-CB22D69360EB}

Fig. 19

\section{Diagnosis}

Within the $C$. degeeri-assemblage, this species is uniquely recognized by a transverse impression (Fig. 5E) across the propodeum, although this character can be faint and difficult to see. Additional useful distinguishing characters are abundant erect pilosity on the promesonotum ( $>$ eight setae) and carinulate or costulate sculpture on the pronotum. In its distribution range C. ramamy sp. nov. can only be confused with $C$. dentata stat. nov., however, the species has longer propodeal spines than the latter 
(C. dentata stat. nov.: SPI 0.01-0.10; C. ramamy sp. nov.: SPI 0.07-0.12), and costulate sculpture on the pronotum (aciculate - reticulate in $C$. dentata stat. nov.). Mean barcode divergences between C. ramamy sp. nov. and other species within the $C$. degeeri-assemblage are 12.7-18.7\%, except with regard to $C$ degeeri, where this is only $4.7 \%$ (Table 3 ). In our analyses, $C$. ramamy sp. nov. renders $C$. degeeri paraphyletic (see comments in description of C. degeeri).

\section{Etymology}

Crematogaster ramamy sp. nov. is named after a Malagasy friend and research assistant, Maminiaina "Mamy" Rajaonarivo, in appreciation of support over several years. The name is treated as a noun in apposition.

\section{Type specimens}

\section{Holotype}

Worker: pinned, CASENT0317780, BLF20074, on low vegetation [imaged on AntWeb]; original locality label: MADG'R: Mahajanga: Antanambezo, $20 \mathrm{~m}-14.87893^{\circ} \mathrm{S}, 47.97712^{\circ} \mathrm{E}, 20$ Apr. 2008, mangrove, Fisher et al., BLF\#; deposited at CASC.

\section{Paratypes}

4 workers, pinned, same collection and locality data as holotype. \#1: CASENT0317781, deposited at SAMC. \#2: CASENT0317782, deposited at MHNG. \#3: CASENT0317783, deposited at MCZC. \#4: CASENT0317784, deposited at UCDC.

\section{Type locality}

MADAGASCAR: Mahajanga: Antanambezo: -14.87893, 47.97712, 20 m, mangrove.

\section{Other material examined}

(BBBC, CASC, PSWC): refer to Supplementary Material 1.

\section{Worker}

\section{Measurements}

$(\mathrm{n}=16)$ [holotype] HW 0.75-1.04 [0.95]; HL 0.71-0.94 [0.90]; EL 0.16-0.22 [0.17]; SL 0.62-0.82 [0.72]; WL 0.86-1.12 [1.03]; SPL 0.06-0.12 [0.09]; PTH 0.12-0.22 [0.18]; PTL 0.22-0.31 [0.27]; PTW 0.220.33 [0.28]; PPL 0.13-0.19 [0.17]; PPW 0.20-0.33 [0.27]; LHT 0.65-0.82 [0.73]; CI 1.05-1.13 [1.05]; OI 0.19-0.24 [0.19]; SI 0.75-0.87 [0.80]; SPI 0.07-0.12 [0.09]; PTHI 0.55-0.76 [0.66]; PTWI 0.95-1.14 [1.04]; PPI 1.36-1.80 [1.58]; LBI 1.27-1.41 [1.40].

\section{Description}

(Fig. 19A, C-D) Small to medium size (HW 0.75-1.04, WL 0.86-1.12).

Masticatory margin of mandibles with four teeth; head shape quadrate, as long or slightly longer than wide (CI 1.05-1.13); posterior margin of head in full-face view laterally rounded or subangular; occipital carinae usually distinct; antennal scapes reaching or surpassing head margin; midline of eyes situated slightly above midline of head in full-face view; eyes moderately large (OI 0.19-0.24) and protruding.

Pronotum laterally angular; promesonotal suture indistinct; mesonotum more or less forming one plane with pronotum, but often the two separated by distinct variation in sculpture (see below); mesonotum usually without posterior face; laterally mesonotum angular to carinate, not distinctly set off from propodeum; metanotal groove shallow, laterally constricted; propodeal spines short, spiniform (SPI 0.07-0.12), usually curved downwards; dorsal face of propodeum short but distinct; posterior face of 
propodeum gently sloping, often with a transverse groove; petiole in dorsal view oval and fairly slender, dorsolaterally rounded, without posterolateral tubercules or denticles; subpetiolar process variable, usually a small dent; postpetiole distinctly bilobed, with a narrow median impression; subpostpetiolar process absent.

Head sculpture aciculate to reticulate; pronotum usually dorsally carinulate and contrasting abruptly with a shiny to aciculate mesonotum; mesopleuron areolate, metapleuron mostly carinulate; dorsal face of propodeum carinulate or reticulate; otherwise sculpture reticulate; face usually with six to ten
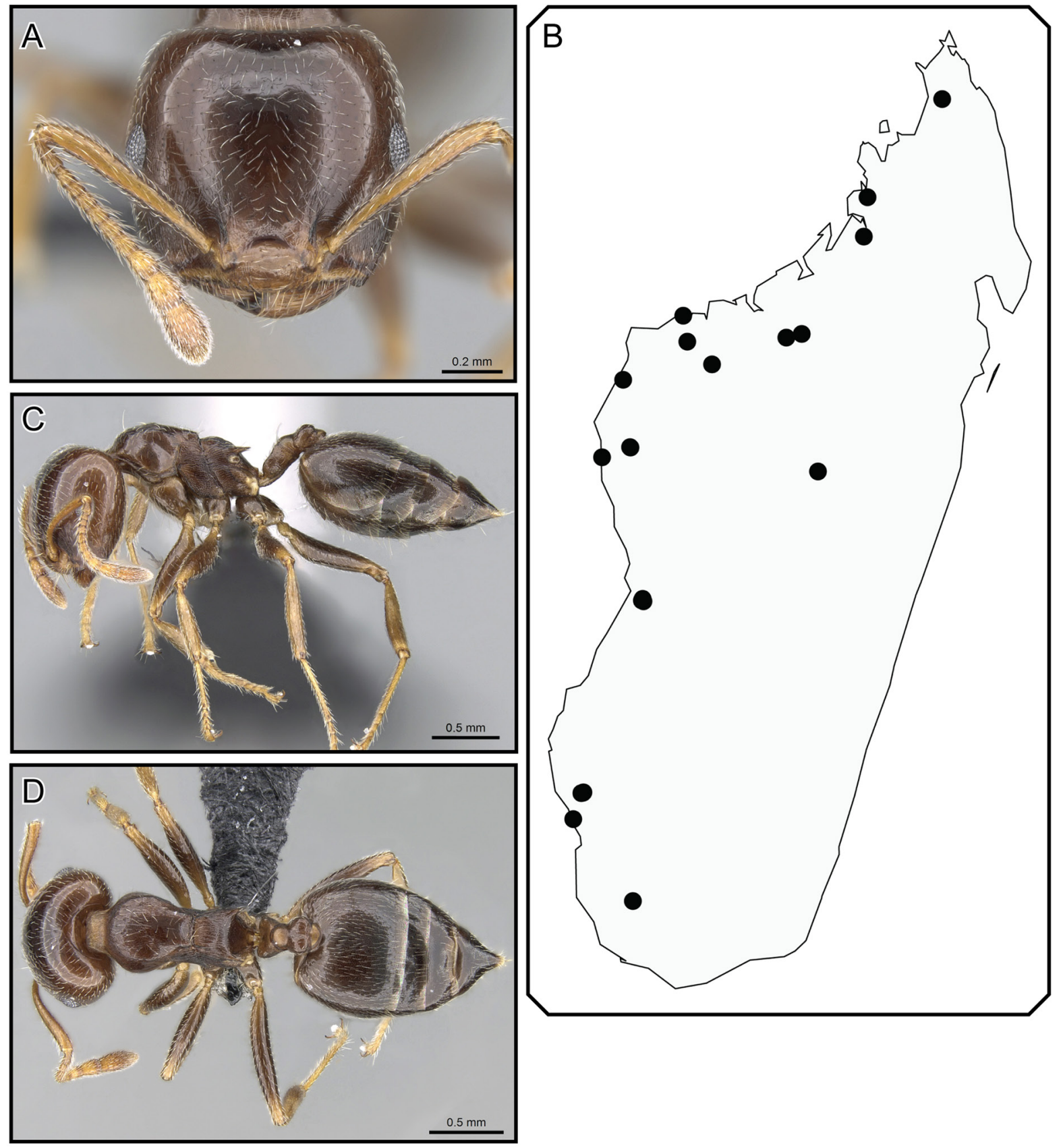

Fig. 19. Crematogaster ramamy sp. nov., worker and distribution. A, C-D. Worker (CASENT0317780). A. Full-face. C. Profile. D. Dorsal. B. Species distribution. 
erect, longer setae, and regular appressed to decumbent pubescence; erect pilosity on promesonotum usually abundant, with > eight long setae; otherwise promesonotum dorsally with regular appressed to subdecumbent pubescence; petiole and postpetiole usually with a pair of short erect, setae posterolaterally, and shorter suberect, decumbent or appressed pubescence; abdominal tergites and sternites four to seven usually with abundant, short, erect pilosity, and abundant appressed to decumbent pubescence throughout. Color reddish brown to dark brown.

\section{Queen and male}

Queens and males of this species are unknown.

\section{Distribution and biology}

MADAGASCAR. Crematogaster ramamy sp. nov. is a species found at low elevations in dry forest, savannah and sometimes mangrove habitats in western and northwestern Madagascar (Fig. 19B). The species further has a disjunct, isolated population at higher altitude in the transitional dry forest of RS Ambohitantely $(1410 \mathrm{~m})$. Crematogaster ramamy sp. nov. usually co-occurs with $C$. dentata stat. nov., C. maina sp. nov. and C. lobata, and at some locations also with C. tricolor stat. rev., C. mafybe sp. nov. and $C$. sewellii. Generally it has been collected nesting arboreally in dead twigs above the ground, and is attracted to arboreal fish baits as strongly during the day as at night.

\section{Comments}

This species has close affinities with $C$. degeeri, and does not appear to be reciprocally monophyletic according to our analysis (Fig. 1). Crematogaster ramamy sp. nov. is however sufficiently distinguishable morphologically from $C$. degeeri, and more importantly seems quite distinct in its distribution and ecological adaptation (dry forest in contrast to montane grassland and woodland for $C$. degeeri).

Crematogaster sewellii Forel, 1891

Figs 20-21

Crematogaster inermis r. sewellii Forel, 1891: 196, pl. 6, fig. 9.

Crematogaster sewellii - Dalla Torre 1893: 86. — Mayr 1895: 138. — Emery 1895: 342.

Crematogaster (Crematogaster) inermis r. sewellii - Wheeler 1922a: 844. — Bolton 1995: 166.

Crematogaster (Acrocoelia) inermis r. sewellii - Emery 1922: 148.

\section{Diagnosis}

Crematogaster sewellii can be identified with the following unique combination of characters: very large eyes (OI 0.24-0.29), antennal scapes usually long (SI 0.77-0.87) and surpassing head margin, propodeal spines reduced to small denticles, and distinct bicoloration with head and thorax yellowish to dark orange and metasoma with abdominal segments four to seven black. Sequence divergence between C. sewellii and the rest of the C. degeeri-assemblage ranges between $12.9 \%$ and $22.2 \%$ (Table 3 ).

\section{Type material examined}

(MHNG): MADAGASCAR: Fianarantsoa (Dr. Besson), six workers. Lectotype worker by present designation: CASENT0101599, lower worker of three workers on one pin, image on AntWeb.

\section{Other material examined}

(BBBC, CASC, MHNG): refer to Supplementary Material 1. 


\section{Worker}

\section{Measurements}

$(\mathrm{n}=20)$ HW 0.72-0.99; HL 0.66-0.89; EL 0.18-0.23; SL 0.56-0.70; WL 0.75-1.01; SPL 0.01-0.04; PTH 0.15-0.21; PTL 0.20-0.28; PTW 0.24-0.33; PPL 0.12-0.19; PPW 0.22-0.29; LHT 0.54-0.73; CI 1.081.13; OI 0.24-0.29; SI 0.77-0.87; SPI 0. 01-0.05; PTHI 0.68-0.87; PTWI 1.07-1.32; PPI 1.54-1.92; LBI $1.30-1.45$.

\section{Description}

(Fig. 20A-C) Small to medium size (HW 0.72-0.99, WL 0.75-1.01).
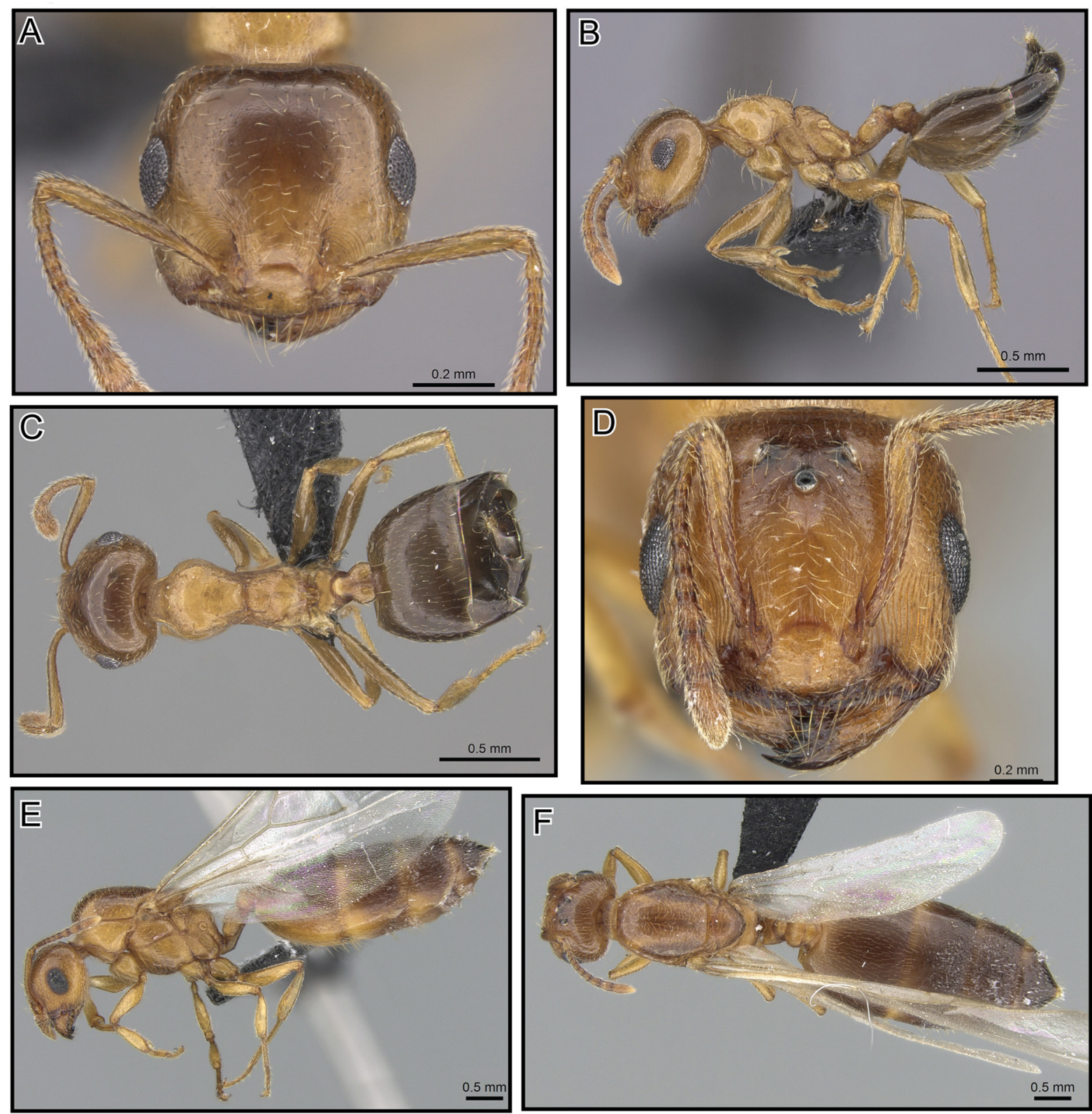

Fig. 20. Crematogaster sewellii Forel, 1891, worker and queen. A-C. Worker (CASENT0120975). A. Full-face. B. Profile. C. Dorsal. D-F. Queen (CASENT0496779). D. Full-face. E. Profile. F. Dorsal. 
Masticatory margin of mandibles with four teeth; head shape fairly quadrate or slightly wider than long (CI 1.08-1.13); posterior margin of head in full-face view laterally subangular or rounded, often slightly medially depressed; occipital carinae distinct; antennal scapes usually reaching or surpassing head margin; midline of eyes situated distinctly above midline of head in full-face view; eyes large (OI 0.24-0.29) and strongly protruding.

Pronotum laterally angular; promesonotal suture indistinct; mesonotum usually forming a flat plane with respect to pronotum; mesonotum usually with a short posterior face; mesonotum angular laterally, posterior face carinate, distinctly set off from propodeum; posterior face of mesonotum sloping steeply into metanotal groove; metanotal groove fairly shallow, laterally constricted and bordered by carinae; propodeal spines reduced to small denticles (SPI 0.01-0.05); dorsal face of propodeum short, but distinct; posterior face of propodeum gently sloping; petiole in dorsal view broadly oval, dorsolaterally rounded, without posterolateral tubercules or denticles; subpetiolar process variable; postpetiole bilobed with a broad median impression; subpostpetiolar process absent.

Head sculpture aciculate; mesosoma, petiole, helcium and postpetiole reticulate; face usually with six to eight erect longer setae, and regular shorter, appressed pubescence; erect pilosity on promesonotum abundant, usually $>$ ten setae; otherwise promesonotum dorsally with regular appressed to subdecumbent pubescence; propodeum laterally with two to four erect setae; petiole and postpetiole usually with a pair of long, erect setae posterolaterally, and shorter appressed pubescence; abdominal tergites and sternites four to seven with abundant short erect pilosity, and regular appressed to decumbent pubescence throughout. More or less bicolored: head, mesosoma, petiole and postpetiole reddish-orange to yellow, rest of metasoma black.

\section{Queen}

\section{Measurements}

$(\mathrm{n}=1)$ HW 1.25; HL 1.18; EL 0.40; SL 0.80; MSNW 1.13; MSNL 1.08; WL 1.97; SPL 0.00; PTH 0.33; PTL 0.42; PTW 0.47; PPL 0.30; PPW 0.47; LHT 0.93; CI 1.06; OI 0.34; SI 0.68; MSNI 1.04; SPI 0.00; PTHI 0.79; PTWI 1.14; PPI 1.59; LBI 2.12.

\section{Description}

(Fig. 20D-F) Small size (HW 1.25, WL 1.97). With worker characters, except as follows.

Masticatory margin of mandibles with five teeth; antennal scapes surpassing level of lateral ocelli; eyes large (OI 0.34), situated at midline of head in full-face view; head shape square (CI 1.06); posterior margin straight.

Mesoscutum in dorsal view as wide as long (MSNI 1.04); dorsal face of propodeum long and set off at right angle to posterior face; propodeal spines or tubercules absent; petiole moderately flared, postpetiole merely impressed behind; subpetiolar process variable.

Sculpture aciculate throughout; face with eight longer erect setae $(n=1 !)$ and abundant shorter erect to suberect pilosity; mesonotum with abundant short and scattered longer erect setae; petiole and postpetiole with dense short suberect pilosity, and postpetiole with additional longer dorso-posterior setae; abdominal tergites and sternites four to seven with abundant erect pilosity and appressed pubescence. Color as in workers; wings clear.

\section{Male}

\section{Measurements}

$(\mathrm{n}=1)$ HW 0.57; HL 0.46; EL 0.22; SL 0.11; MSNW 0.69; MSNL 0.61; WL 1.24; SPL 0.00; PTH 0.17; PTL 0.22; PTW 0.22; PPL 0.14; PPW 0.24; LHT 0.60; CI 1.23; OI 0.47; SI 0.24; MSNI 1.14; SPI 0.00; PTHI 0.77; PTWI 0.99; PPI 1.64; LBI 2.07. 


\section{Description}

(Fig. 21A, C) Small (HW 0.57, WL 1.24).

Mandibles very short and reduced, blunt and edentate; clypeus fairly flat; eyes fairly large (OI 0.47) and protruding, midline of eyes situated well below midline of head, almost extending to clypeal margin; antennae 12-segmented; head wider than long (CI 1.23); in full-face view ocellar triangle situated at posterior head margin and elevated with respect to rest of face; occipital carinae distinct.

Mesoscutum in dorsal view as wide as long (MSNI 1.14), scutellum in dorsal view broadly tapering from anterior to posterior end, dorsoposterior part rounded; metanotum not protruding from below scutellum; dorsal face of propodeum almost as long as posterior face; propodeal spines absent; petiole in dorsal view oval, laterally rounded, in lateral view anteriorly greatly tapering; subpetiolar process absent; postpetiole fairly flat and short, lacking median impression.

Head sculpture reticulate; mesoscutum with very fine longitudinal carinulae; otherwise sculpture more or less shiny to aciculate; regular appressed to decumbent pubescence, and a few longer setae on face, mesoscutum, and scutellum; remaining pilosity as in queen. Color brown.
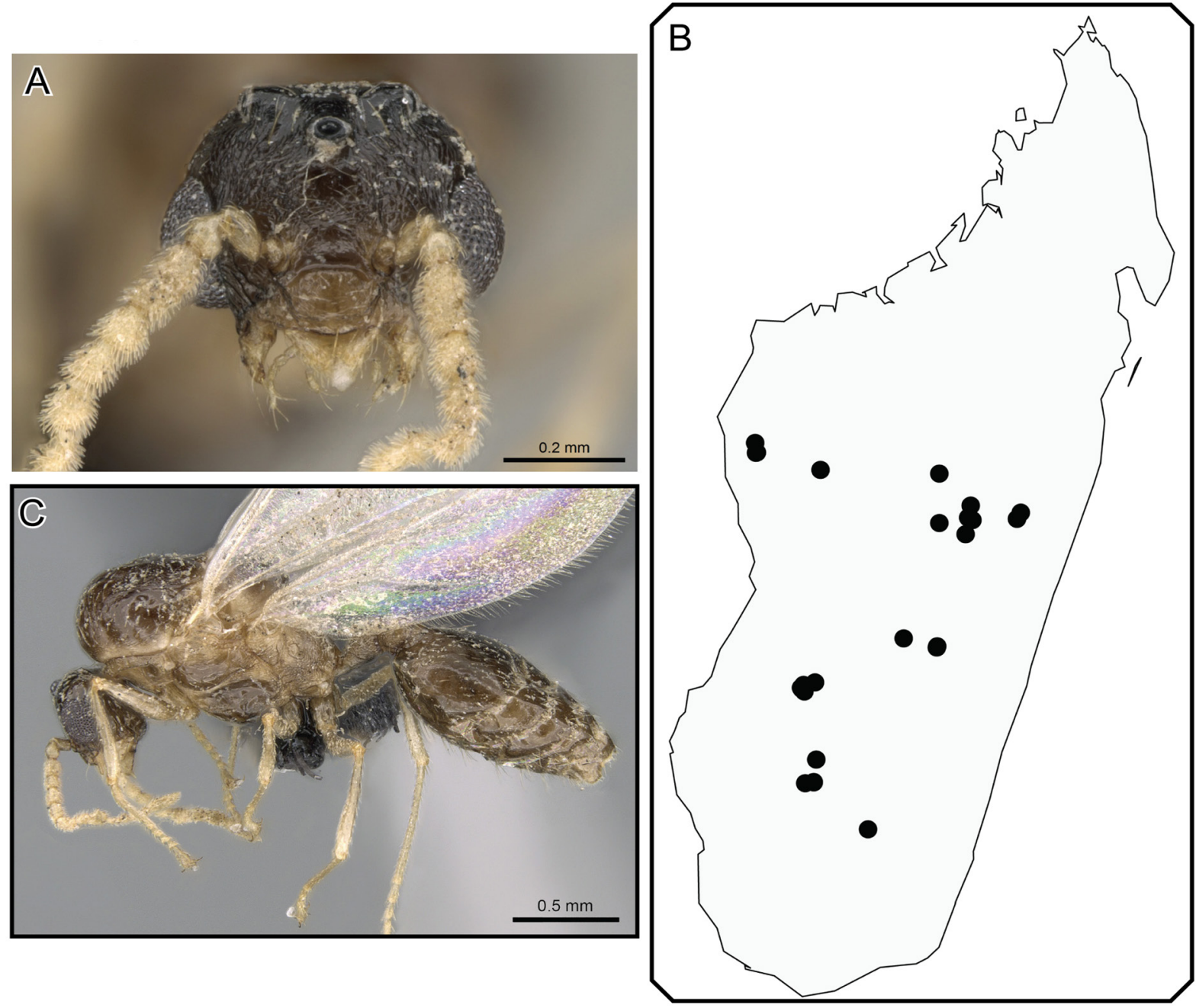

Fig. 21. Crematogaster sewellii Forel, 1891, male and distribution. A, C. Male (CASENT0317761). A. Full-face. C. Profile. B. Species distribution. 


\section{Distribution and biology}

MADAGASCAR. Crematogaster sewellii is commonly found in high-altitude grassland, savannah and woodland habitat in the central and south-central highland region $(800-1500 \mathrm{~m})$, and at lower elevations (160-600 m) in the Makay massif and the RF Beanka in western Madagascar (Fig. 21B). The species often co-occurs with $C$. mafybe sp. nov. and $C$. degeeri, and otherwise with all other species of the $C$. degeeri-assemblage except $C$. malahelo sp. nov. This species appears to nest exclusively on the ground, either in the soil or under stones.

Crematogaster tricolor Gerstäcker, 1859 stat. rev.

Figs 22-23

Crematogaster tricolor Gerstäcker, 1859: 263 (w.) [also described as new by Gerstäcker 1862: 515].

Crematogaster tricolor - Forel 1887: 388 (q.m.).

Crematogaster tricolor, junior synonym of Crematogaster castanea - Mayr 1907: 16. - Emery 1915: 12. - Wheeler 1922a: 830.

Crematogaster castanea tricolor - Arnold 1920: 489. - Emery 1922: 145. — Santschi 1926: 213.

C. (Acrocoelia) tricolor - Emery 1922: 145.

\section{Diagnosis}

The tricoloration of this species (see Fig. 22B, C) serves to uniquely identify this species from all other Crematogaster species in the $C$. degeeri-assemblage. Beyond the color, the mostly very long propodeal spines (SPI 0.10-0.26) are also useful diagnostic characters. Sequence divergence between C. tricolor stat. rev. and the remaining species ranges from 16.6-22.2\% (Table 3).

\section{Type material examined}

(ZMHB): MOZAMBIQUE: Tete (S. Peters), one worker; lectotype worker by present designation: CASENT0104590, image on AntWeb.

\section{Other material examined}

(BBBC, CASC, MCZC, PSWC, ZMHB): refer to Supplementary Material 1.

\section{Worker}

\section{Measurements}

$(\mathrm{n}=20)$ HW 0.71-1.27; HL 0.65-1.14; EL 0.16-0.26; SL 0.54-0.88; WL 0.73-1.37; SPL 0.08-0.30; PTH 0.16-0.30; PTL 0.20-0.37; PTW 0.23-0.47; PPL 0.11-0.25; PPW 0.18-0.39; LHT 0.54-0.96; CI 1.101.19; OI 0.21-0.25; SI 0.77-0.83; SPI 0.10-0.26; PTHI 0.60-0.80; PTWI 1.07-1.26; PPI 1.46-1.85; LBI $1.30-1.45$.

\section{Description}

(Fig. 22A-C) Small to large size (0.71-1.27, WL 0.73-1.37), usually large.

Masticatory margin of mandibles with four teeth; head shape usually wider than long (CI 1.10-1.19); posterior margin of head in full-face view laterally subangular, medially depressed; occipital carinae distinct; antennal scapes reaching or surpassing head margin; midline of eyes situated above midline of head in full-face view; eyes fairly large (OI 0.21-0.25) and strongly protruding.

Pronotum laterally angular; promesonotal suture indistinct; mesonotum often slightly raised with respect to pronotum, and with a median tubercule or carinae; mesonotum usually with a short posterior face; laterally mesonotum angular, posterior face carinate, distinctly set off from propodeum; posterior 
face of mesonotum sloping steeply into metanotal groove; metanotal groove fairly shallow, laterally constricted and bordered by carinae; propodeal spines short to medium-sized (SPI 0.10-0.26), straight and moderately diverging; dorsal face of propodeum as long as posterior face; posterior face of propodeum gently sloping; petiole in dorsal view moderately flared and concave, dorsolaterally rounded, without posterolateral tubercules or denticles; subpetiolar process variable; postpetiole narrowly bilobed with a sharp median impression; subpostpetiolar process absent.

Head and mesosomal sculpture areolate; petiole, helcium and postpetiole dorsally and ventrally reticulate; erect pilosity on face scarce, usually less than four erect longer setae, and regular, shorter, appressed pubescence; erect pilosity on promesonotum scarce, usually only two to four humeral setae; otherwise promesonotum dorsally with regular appressed to subdecumbent pubescence; petiole and postpetiole usually with a pair of short, erect setae posterolaterally, and shorter appressed pubescence; abdominal tergites four to seven with sparse, short, erect pilosity, more abundant and longer on sternites four to seven, and abundant appressed to decumbent pubescence throughout. Distinctly tricolored: Head and mesosoma reddish-orange, first half of abdominal segment four yellow, rest black.

\section{Queen}

\section{Measurements}

$(\mathrm{n}=8)$ HW 1.61-1.77; HL 1.38-1.49; EL 0.44-0.49; SL 0.98-1.10; MSNW 1.20-1.45; MSNL 1.32-1.46; WL 2.43-2.69; SPL 0.00-0.04; PTH 0.38-0.44; PTL 0.42-0.57; PTW 0.57-0.67; PPL 0.36-0.40; PPW 0.57-0.64; LHT 1.13-1.30; CI 1.14-1.21; OI 0.31-0.33; SI 0.69-0.74; MSNI 0.85-1.04; SPI 0.00-0.02; PTHI 0.72-1.05; PTWI 1.18-1.53; PPI 1.41-1.69; LBI 1.98-2.30.

\section{Description}

(Figs 22D-F) Medium size (HW 1.61-1.77, WL 2.43-2.69). With worker characters, except as follows.

Masticatory margin of mandibles with five teeth; antennal scapes reaching or surpassing level of lateral ocelli; eyes fairly large (OI 0.31-0.33), situated at midline of head in full-face view; head distinctly wider than long (CI 1.14-1.21), tapering from anterior to posterior margin; posterior margin straight.

Mesoscutum in dorsal view longer than wide, or as wide as long (MSNI 0.85-1.04); dorsal face of propodeum short; propodeal tubercules present; petiole moderately flared, postpetiole merely impressed behind; subpetiolar process variable.

Sculpture carinulate on face and propodeum, otherwise aciculate throughout; face usually with $>$ six longer erect setae and abundant shorter erect to suberect pilosity; mesonotum with abundant short and scattered longer erect setae; petiole and postpetiole with dense, short, suberect pilosity, and postpetiole often with additional, longer dorso-posterior setae; abdominal tergites and sternites four to seven with abundant appressed pilosity and sparse, short, erect pilosity. Tricoloration is similar to workers, but less distinct; wings clear.

\section{Male}

\section{Measurements}

$(\mathrm{n}=3)$ HW 0.73-0.82; HL 0.54-0.60; EL 0.27-0.31; SL 0.10-0.15; MSNW 0.80-0.95; MSNL 0.77-0.91; WL 1.40-1.78; SPL 0.00; PTH 0.21-0.24; PTL 0.25-0.29; PTW 0.27-0.31; PPL 0.18; PPW 0.28-0.35; LHT 0.73-0.91; CI 1.32-1.36; OI 0.50-0.53; SI 0.19-0.26; MSNI 1.01-1.04; SPI 0.00; PTHI 0.80-0.83; PTWI 1.05-1.10; PPI 1.59-1.91; LBI 1.92-2.00.

\section{Description}

(Fig. 23A, C) Small (HW 0.73-0.82, WL 1.40-1.78). 
Mandibles short (not touching when closed) with two regular-sized teeth; clypeus fairly flat; eyes large (OI 0.50-0.53) and protruding, midline of eyes situated well below midline of head, almost extending to clypeal margin; antennae 12-segmented; head wider than long (CI 1.32-1.36); in full-face view ocellar triangle situated at posterior head margin and elevated with respect to rest of face; occipital carinae distinct.

Mesoscutum in dorsal view as wide as long (MSNI 1.01-1.04); scutellum in dorsal view broadly tapering from anterior to posterior end, dorsoposterior part rounded; metanotum protruding from below
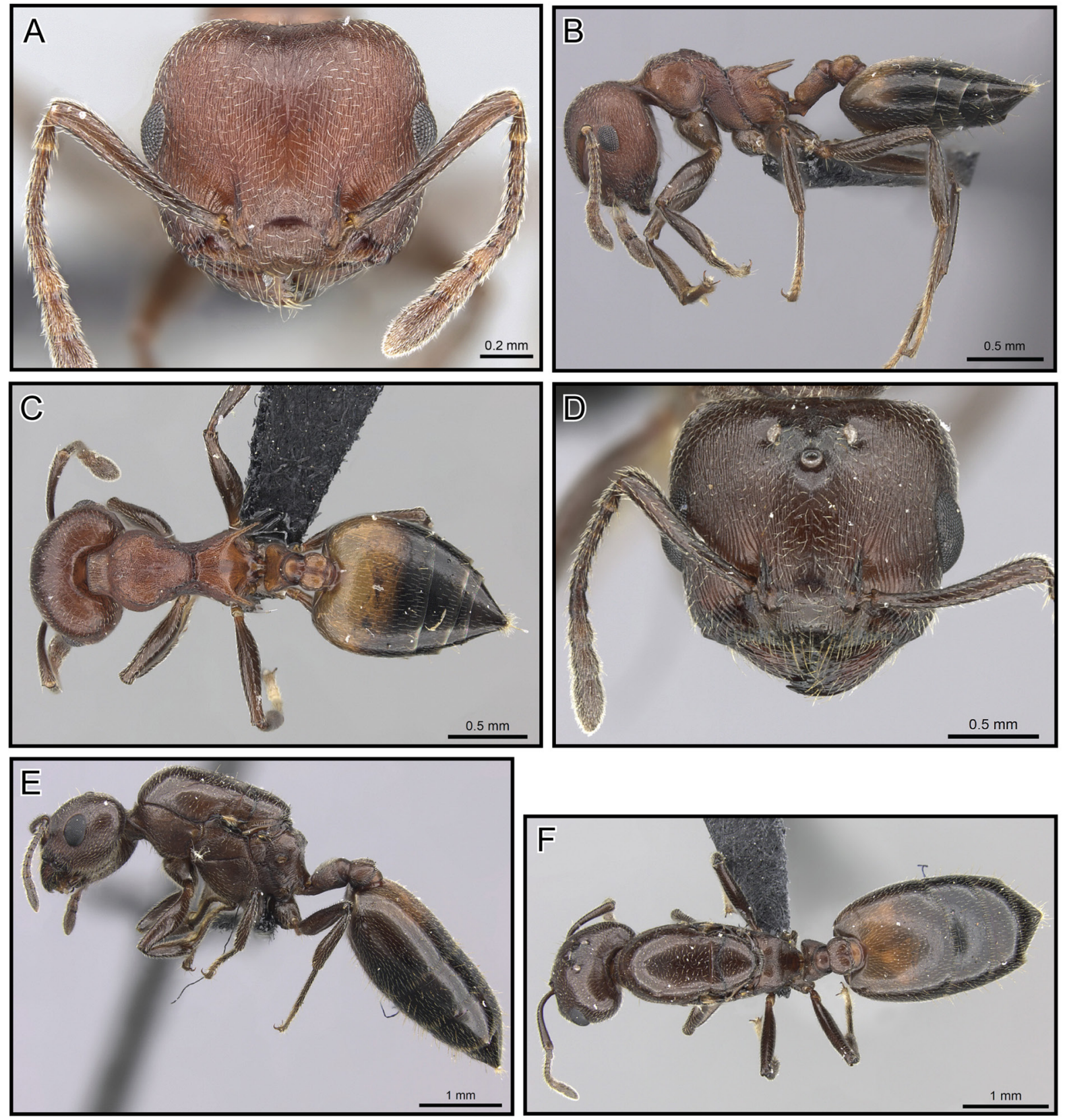

Fig. 22. Crematogaster tricolor Gerstäcker, 1859 stat. rev., worker and queen. A-C. Worker (CASENT0120944). A. Full-face. B. Profile. C. Dorsal. D-F. Queen (CASENT0120949). D. Full-face. E. Profile. F. Dorsal. 
scutellum; dorsal face of propodeum almost as long as posterior face; propodeal spines absent; propodeal spiracle situated in a margined depression on propodeum; petiole in dorsal view oval, laterally rounded, in lateral view anteriorly greatly tapering; subpetiolar process absent; postpetiole fairly flat and short, lacking median impression.

Head sculpture reticulate to carinulate; mesoscutum with very fine longitudinal carinulae; otherwise sculpture more or less shiny to aciculate; abundant, short, erect pilosity, but very few longer setae on face, mesoscutum, and scutellum; remaining pilosity as in queens. Color brown.

\section{Distribution and biology}

MADAGASCAR, COMOROS, MAYOTTE, MOZAMBIQUE [East Africa?]. Crematogaster tricolor stat. rev. was originally described from Mozambique (see comments), but we limit ourselves here to its description in the Malagasy region. The species has been recently collected in scrublands of coastal areas and urban and disturbed habitats in northern, eastern and central Madagascar, and in Comoros and

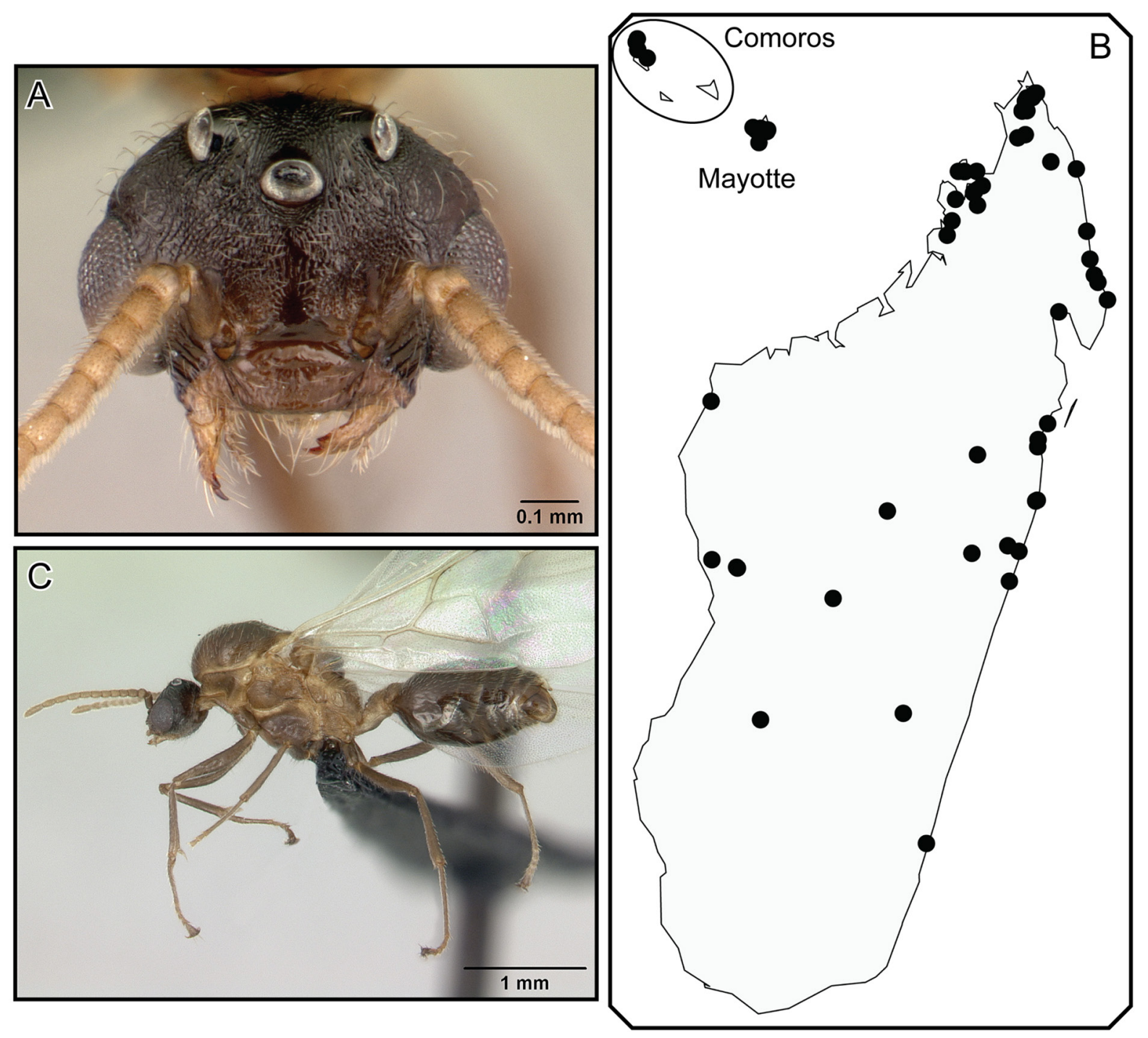

Fig. 23. Crematogaster tricolor Gerstäcker, 1859 stat. rev., male and distribution. A, C. Male (CASENT0136505). A. Full-face. C. Profile. B. Species distribution. 
Mayotte, occuring at elevations from $0 \mathrm{~m}$ to $1240 \mathrm{~m}$ (Fig. 23B). Collections in native habitat have been made in dry forest and littoral forest in northern Madagascar. Crematogaster tricolor stat. rev. co-occurs usually with $C$. dentata stat. nov., $C$. maina sp. nov. and $C$. lobata, and at a few localities also with $C$. sewellii, C. mafybe sp. nov. and C. masokely sp. nov. Very few colonies have been collected from dead twigs above ground, indicating that this species has an arboreal nesting habit.

\section{Comments}

We raised Crematogaster tricolor from subspecies to species status considering the following points.

1) We examined the syntype specimens of $C$. castanea tricolor in ZMHB and found them to be conspecific to our recent collections from the Malagasy region.

2) We examined images of syntype specimens of $C$. castanea Smith, which are available on AntWeb (CASENT0102953), and found them to be a good match for specimens one of us (B. Blaimer) had received from a colleague collected in Mozambique.

3) Both this $C$. castanea material and the $C$. tricolor material from Madagascar were included in a molecular biogeographic study on Crematogaster ants (Blaimer 2012b), where the sequences of these specimens proved to be divergent and clearly not conspecific.

In this study, we limit ourselves to describing the presence of $C$. tricolor stat. rev. in the Malagasy region, although the species should still occur on the African mainland. Recent ant collections from Mozambique are poor, and historic collections are in such disarray for this genus that they would be only manageable to cite in a large scale taxonomic project on African Crematogaster, which is not the focus of this project. Moreover, apart from C. tricolor stat. rev., there are 18 further subspecies currently listed by Bolton (2012) for C. castanea, described from all over East and Central Africa, that would need to be revised during such a comprehensive undertaking.

The predominant occurrence of this species in urban and disturbed habitats, and the two distinct clades in the mitochondrial data with different affinities to the Comoros and Mayotte populations (Fig. 2, see above) suggest that this species was only recently introduced to Madagascar.

\section{Discussion}

In this study, we have presented a revision of the $C$. degeeri-species-assemblage based on morphological and molecular data, and describe and re-describe a total of 12 species, seven of which are new to science. In four cases (C. degeeri, C. ramamy sp. nov., C. maina sp. nov., C. dentata stat. nov.) the mitochondrial data has not lent unequivocal support for our preformed species hypotheses based on morphology. We have reported on this in the context of species descriptions, but it seems worthwhile to reiterate and elaborate briefly on the potential causes of these discrepancies.

In the case of C. degeeri and C. ramamy sp. nov. the data indicate that these species are not reciprocally monophyletic with respect to each other (Fig. 1). These two species are indeed morphologically very similar, with differences mainly in promesonotal structure and sculpture. They could represent the products of a very recent, or even ongoing speciation event, and given their diverging habitat preferences (mainly low elevation dry forest for $C$. ramamy sp. nov. and mainly montane habitats for $C$. degeeri), this process may well be environmentally driven. A different issue to consider is the possibility that pseudogene (Nuclear mitochondrial DNA [NUMTs], Buhay 2009; Song et al. 2008) sequences are responsible for this pattern. Although we found no evidence of NUMTs in the relevant sequences, such as heteroplasmy or stop codons, we are reluctant to give much credence to potentially questionable results. The same line of reasoning has led us to ignore the non-monophyly of the individuals sampled for C. maina sp. nov. (see Figs 1 and 2A). These results could either represent a highly divergent population on Comoros (and Nosy Faly), or be caused by spurious data. In any case, the $13.3 \%$ sequence divergence between Comoros and Madagascar in C. maina sp. nov. remains within the limits of sequence divergence found 
for other widespread species of Crematogaster in Madagascar, for example in C. kelleri (Blaimer \& Fisher 2013) or C. ranavalonae (Blaimer 2012a).

Similarly, our analyses have lent virtually no support to grouping the Mauritius and the Madagascar population of $C$. dentata stat. nov. (Fig. 1 and Fig. 2B). Here the sequence divergence is somewhat smaller (11.2\%) than in C. maina sp. nov. Despite the fact that Mauritius is separated by almost $900 \mathrm{~km}$ of open ocean from Madagascar and gene flow between the two populations is probably non-existent, this result seems to indicate a low level of mitochondrial genetic divergence. Since reproductive isolation between these disjunct populations cannot be tested, the question of whether to separate the populations into two species or not is a rather philosophical one, and our decision must be somewhat arbitrary.

The limitations of our molecular data are obvious, as only a short stretch of the barcoding region was sampled, and we wish to avoid drawing poorly supported conclusions about phylogeographic relationships between regions. The pattern seen in Crematogaster tricolor stat. rev. (as discussed above, Fig. 2C) is nonetheless quite intriguing and could be evidence of more frequent dispersal events of this species between Madagascar and the Indian Ocean Islands. This may also indicate that this species could be a very recent addition to the Malagasy fauna, though this hypothesis currently lacks sufficient data for support. For the reasons already stated above, we had to further ignore the potential that any of the widespread species treated here could also occur on the African mainland. For $C$. tricolor stat. rev., $C$. maina sp. nov. and $C$. dentata stat. nov. this possibility should be considered in the future when more specimens and data on East African Crematogaster become available.

To conclude, this revision solves the last piece of the taxonomic puzzle of Crematogaster ants in the Malagasy region. Prior to the start of this revisionary work, there were 20 species and numerous subspecies described for this genus and region. With the publication of this study we have raised the total number of species for Crematogaster in Madagascar to 34, of which 19 were new to science (Blaimer 2010; Blaimer 2012c). With each of these taxonomic papers it has become clearer that even in such a seemingly conspicuous ant genus as Crematogaster, a large part of the species diversity actually consists of rare, endemic species with very restricted distributions. In other parts of the world much of this diversity surely remains hidden and undescribed, and we hope that the present study can help provide guidelines for future, much-needed taxonomic work on Crematogaster ants.

\section{Acknowledgements}

We are greatly indebted to the Malagasy ant team for generating the extensive specimen data that formed the basis of this revision; BBB would further like to acknowledge the help of field assistants M. Rasoanarivo, A. Rakotomalala, T. Razafindrabe and E.H. Lokensgard. In addition, we received invaluable access to and loans of specimens from the following curators: B. Merz, F. Koch, R. Poggi, S. Cover, G. Alpert and P.S. Ward. Special thanks to Estella Ortega, Michele Esposito and Shannon Hartmann for creating and processing the Automontage images used for this article. A.M. Smith generously provided access to cytochrome oxidase I data, which we greatly appreciate. We thank the following institutions for delivering authorizations for the capture, collection and exportation of ants COMOROS: Centre National de Documentation et de Recherche Scientifique (CNDRS); ESPARSE: Terres Australes et Antarctiques Françaises (TAAF), Direction de la Conservation du Patrimoine Naturel (DCPN); MADAGASCAR: Ministère de l' Environnement et des Forêts and the Madagascar National Parks; MAURITIUS: Forestry Service and National Parks and Conservation Service. This project was supported by a National Geographic grant No. 8429-08 and National Science Foundation under Grant No. DEB-0072713, DEB-0344731, and DEB-0842395 to BLF; BBB was supported during field work by a National Science Foundation Grant No. DEB-1107515 (awarded to BBB \& P.S. Ward), a National Geographic Waitt Grant No. W173-11 and the Lewis \& Clark Fund for Exploration and Field Research. 
BLAIMER B. \& FISHER B.L., The Crematogaster degeeri-species-assemblage

\section{References}

AntWeb. Available from http://www.antweb.org [accessed 20 Mar. 2013]

Arnold G. 1920. A monograph of the Formicidae of South Africa. (Myrmicinae). Annals of the South African Museum 14 (4): 403-578.

Blaimer B.B. 2010. Taxonomy and natural history of the Crematogaster (Decacrema) group in Madagascar. Zootaxa 2714: 1-39.

Blaimer B.B. 2012a. Untangling complex morphological variation: taxonomic revision of the subgenus Crematogaster (Oxygyne) in Madagascar, with insight into the evolution and biogeography of this enigmatic ant clade (Hymenoptera: Formicidae). Systematic Entomology 37 (2): 240-260. http://dx.doi. org/10.1111/j.1365-3113.2011.00609.x

Blaimer B.B. 2012b. Acrobat ants go global - Origin, evolution and systematics of the genus Crematogaster (Hymenoptera: Formicidae). Molecular Phylogenetics and Evolution 65 (2): 421-436. http://dx.doi.org/10.1016/j.ympev.2012.06.028

Blaimer B.B. 2012c. Taxonomy and species-groups of the subgenus Crematogaster (Orthocrema) in the Malagasy region (Hymenoptera, Formicidae). ZooKeys 199: 23-70. http://dx.doi.org/10.3897/ zookeys.199.2631

Blaimer B.B. 2012d. A subgeneric revision of Crematogaster and discussion of regional species-groups (Hymenoptera: Formicidae). Zootaxa 3482: 47-67.

Blaimer B.B. \& Fisher B.L. 2013. How much variation can one ant species hold? Species delimitation in the Crematogaster kelleri-group in Madagascar. PLoS ONE 8 (7): e68082. http://dx.doi.org/10.1371/ journal.pone.0068082

Bolton B. 1995. A new general catalogue of the ants of the world. Harvard University Press, Cambridge, Massachusetts.

Bolton B. 2012. Bolton's Catalogue and Synopsis [online]. Version January 2012. Available from http:// gap.entclub.org/archive/Bolton_NGC_2012_JAN.pdf [accessed 10 Dec. 2012]

Brown W.L. 1973. A comparison of the Hylean and Congo-West African rain forest ant faunas. In: Meggers B.J., Ayensu E.S. \& Duckworth W.D (eds) Tropical forest ecosystems in Africa and South America: a comparative review: 161-185. Smithsonian Institution Press, Washington, DC.

Buhay J.E. 2009. "COI-like" sequences are becoming problematic in molecular systematic and DNA barcoding studies. Journal of Crustacean Biology 29 (1): 96-110. http://dx.doi.org/10.1651/08-3020.1

Dalla Torre K.W. 1893. Catalogus Hymenopterorum hucusque descriptorum systematicus et synonymicus. Vol. 7: Formicidae (Heterogyna). G. Engelmann, Leipzig.

Donisthorpe H. 1946. The ants (Hym. Formicidae) of Mauritius. Annals and Magazine of Natural History 13 (97): 25-35. http://dx.doi.org/10.1080/00222934608654518

Edgar R.C. 2004. MUSCLE: multiple sequence alignment with high accuracy and high throughput. Nucleic Acids Research 32 (5): 1792-1797. http://dx.doi.org/10.1093/nar/gkh340

Emery C. 1895. Mission scientifique de M. Ch. Alluaud dans le territoire de Diego-Suarez (Madagascarnord) (Avril-Août 1893). Formicides. Annales de la Société Entomologique de Belgique 39: 336-345.

Emery C. 1915. Formiche raccolte nell'Eritrea dal Prof. F. Silvestri. Bollettino del Laboratorio di Zoologia Generale e Agraria della Reale Scuola Superiore d'Agricoltura 10, Portici. 
Emery C. 1922. Hymenoptera, fam. Formicidae, subfam. Myrmicinae. Genera Insectorum 174B, L. Desmet-Verteneuil, Bruxelles.

Fisher B.L. 2003. Formicidae, ants. In: Goodman S.M. \& Benstead J.P. (eds) The Natural History of Madagascar: 811-819. The University of Chicago Press, Chicago.

Fisher B.L. 2005. A model for a global inventory of ants: A case study in Madagascar. Proceedings of the California Academy of Sciences 56 (8), Supplement I: 86-97.

Fisher B.L. 2008. Les Fourmis. In: Goodman S.M. (ed) Paysage naturels et biodiversité de Madagascar: 249-271. Publications scientifiques du Muséum, Muséum national d'Histoire naturelle, Paris.

Forel A. 1886. Diagnoses provisoires de quelques espèces nouvelles de fourmis de Madagascar, récoltées par M. Grandidier. Annales de la Société Entomologique de Belgique, Comptes-rendus de Séance 30.

Forel A. 1887. Fourmis récoltées à Madagascar par le Dr. Conrad Keller. Mitteilungen der Schweizer Entomologischen Gesellschaft 7: 381-389.

Forel A. 1891. Les Formicides. In: Grandidier A. (ed.) Histoire naturelle des Hyménoptères. Deuxième partie. Histoire physique, naturelle et politique de Madagascar 22, Imprimerie nationale, Paris. http:// dx.doi.org/10.5962/bhl.title.1599

Forel A. 1907. Ameisen von Madagaskar, den Comoren und Ostafrika. Wissenschaftliche Ergebnisse. Reise in Ostafrika 2: 75-92.

GARLI Web Service. GARLI v2.0. Available from http://molecularevolution.org [accessed 3 Mar. 2013]

Gautier L. \& Goodman S.M. 2003. Introduction to the Flora of Madagascar. In: Goodman S.M. \& Benstead J.P. (eds) The Natural History of Madagascar: 229-250. The University of Chicago Press, Chicago.

Gerstäcker A. 1859. Hr. Peters berichtete über sein Reisewerk, von dem die Insecten bis zum 64., die Botanik bis zum 34. Bogen gedruckt sind und theilte den Schluss der Diagnosen der von Hrn. Dr. Gerstäcker bearbeiteten Hymenopteren mit. Monatsberichte der Königlichen Preussischen Akademie der Wissenschaften zu Berlin 1858: 261-264.

Gerstäcker A. 1862. Hymenoptera Hautflügler. In: Peters W.C.H. (ed.) Naturwissenschaftliche Reise nach Mossambique auf Befehl seiner Majestät des Königs Friedrich Wilhelm IV in den Jahren 1842 bis 1848 ausgeführt: 439-526. G. Reimer, Berlin.

Hita-Garcia F. \& Fisher B.L. 2011. The ant genus Tetramorium Mayr (Hymenoptera: Formicidae) in the Malagasy region: Introduction, definition of species groups, and revision of the T. bicarinatum, $T$. obesum, T. sericeiventre and T. tosii species groups. Zootaxa 3039: 1-72.

Hita-Garcia F. \& Fisher B.L. 2012. The ant genus Tetramorium Mayr (Hymenoptera: Formicidae) in the Malagasy region - taxonomic revision of the T. kelleri and T. tortuosum species groups. Zootaxa 3592: 1-85.

Hosoishi S. \& Ogata K. 2009. A taxonomic revision of the Asian endemic subgenus Physocrema of the genus Crematogaster (Hymenoptera: Formicidae). Zootaxa 2062: 15-36.

Hosoishi S. \& Ogata K. 2012. Revision of the Crematogaster brevis complex in Asia (Hymenoptera: Formicidae). Zootaxa 3349: 18-30.

International Commission on Zoological Nomenclature 1999. International Code of Zoological Nomenclature version 4. Available from: http://www.nhm.ac.uk/hosted-sites/iczn/code/ [accessed 27 Oct. 2012] 
BLAIMER B. \& FISHER B.L., The Crematogaster degeeri-species-assemblage

Lanfear R., Calcott B., Ho S.Y.W. \& Guindon S. 2012. PartitionFinder: Combined Selection of Partitioning Schemes and Substitution Models for Phylogenetic Analyses. Molecular Biology and Evolution 29 (6): 1695-1701. http://dx.doi.org/10.1093/molbev/mss020

LaPolla J.S., Cheng C.H. \& Fisher B.L. 2010. Taxonomic revision of the ant (Hymenoptera: Formicidae) genus Paraparatrechina in the Afrotropical and Malagasy Regions. Zootaxa 2387: 1-27.

Longino J.T. 2003. The Crematogaster (Hymenoptera, Formicidae, Myrmicinae) of Costa Rica. Zootaxa 151: $1-150$.

Mayr G. 1895. Afrikanische Formiciden. Annalen des Kaiserlich-Königlichen Naturhistorischen Museums in Wien 10 (2): 124-154.

Mayr G. 1907. 2. Formicidae. In: Sjöstedt Y. (ed.) 1907-1910 Wissenschaftliche Ergebnisse der Schwedischen Zoologischen Expedition nach dem Kilimandjaro, dem Meru und umgebenden Massaisteppen Deutsch-Ostafrikas 1905-1906. 2. Band, Abt. 8: 7-24. Königlich-Schwedischen Akademie der Wissenschaften, Stockholm.

Miller M.A., Pfeiffer W. \& Schwartz T. 2010. Creating the CIPRES Science Gateway for inference of large phylogenetic trees. In: Proceedings of the Gateway Computing Environments Workshop (GCE): 1-8. New Orleans, LA. http://dx.doi.org/10.1109/GCE.2010.5676129

National Geospatial-Intelligence Agency. GEOnet Names Server. Available from http://earth-info.nga. mil/gns/html/index.html [accessed 25 Oct. 2012]

Nylander J.A., Wilgenbusch J.C., Warren D.L. \& Swofford D.L. 2008. AWTY (are we there yet?): a system for graphical exploration of MCMC convergence in Bayesian phylogenetics. Bioinformatics 24 (4): 581-583. http://dx.doi.org/10.1093/bioinformatics/btm388

R Development Core Team 2008. R: A Language and Environment for Statistical Computing. R Foundation for Statistical Computing, Vienna, Austria. Available from http://www.R-project.org [accessed 15 Mar. 2013]

Rambaut A. 2006-2009. FigTree: tree figure drawing tool. Version 1.3.1. Available from http://tree.bio. ed.ac.uk/software/figtree/ [accessed 22 Oct. 2012]

Rambaut A. \& Drummond A.J. 2007. Tracer v1.4 [upated to v1.5]. Available from http://beast.bio.ed.ac. uk/Tracer. [accessed 20 Oct. 2012]

Ronquist F., Teslenko M., van der Mark P., Ayres D.L., Darling A., Höhna S., Larget B., Liu L., Suchard M.A, Huelsenbeck J.P. 2012. MrBayes 3.2: Efficient Bayesian phylogenetic inference and model choice across a large model space. Systematic Biology 61: 539-542. http://dx.doi.org/10.1093/sysbio/sys029

Santschi F. 1919. Fourmis nouvelles éthiopiennes. Revue Zoologique Africaine 6 (3): 229-240.

Santschi F. 1926. Description de nouveaux Formicides éthiopiens (III ${ }^{\mathrm{eme}}$ partie). Revue Zoologique Africaine 13: 207-267.

Santschi F. 1928. Descriptions de nouvelles fourmis éthiopiennes (quatrième note). Revue de Zoologie et de Botanique Africaines 16: 54-69.

Santschi F. 1930. Description de Formicides éthiopiens nouveaux ou peu connus. V. Bulletin et Annales de la Société Entomologique de Belgique 70: 49-77.

Schatz G.E. \& Lescot M. 2012. Gazetteer to Malagasy botanical collecting localities. Available from http://www.mobot.org/mobot/research/madagascar/gazetteer/ [accessed 25 Oct. 2012]

Schulz W.A. 1906. Spolia hymenopterologica. Junfermannsche Buchhandlung, Paderborn. 
Smith M.A. \& Fisher B.L. 2009. Invasions, DNA barcodes, and rapid biodiversity assessment using ants of Mauritius. Frontiers in Zoology 6 (31). http://dx.doi.org/10.1186/1742-9994-6-31

Song H., Buhay J.E., Whiting M.F. \& Crandall K.A. 2008. Many species in one: DNA barcoding overestimates the number of species when nuclear mitochondrial pseudogenes are coamplified. Proceedings of the National Academy of Sciences of the United States of America 105 (36): 1348613491. http://dx.doi.org/10.1073/pnas.0803076105

Tamura K. \& Nei M. 1993. Estimation of the number of nucleotide substitutions in the control region of mitochondrial DNA in humans and chimpanzees. Molecular Biology and Evolution 10 (3): 512-526.

Tamura K., Peterson D., Peterson N., Stecher G., Nei M. \& Kumar S. 2011. MEGA5: Molecular Evolutionary Genetics Analysis Using Maximum Likelihood, Evolutionary Distance, and Maximum Parsimony Methods. Molecular Biology and Evolution 28 (10): 2731-2739. http://dx.doi.org/10.1093/ molbev/msr121

Ward P.S. 2007. Phylogeny, classification, and species-level taxonomy of ants (Hymenoptera: Formicidae). Zootaxa 1668: 549-563.

Ward P.S. 2010. Taxonomy, Phylogenetics and Evolution. In: Lach L., Parr C.L. \&. Abott K.L (eds) Ant Ecology: 3-17. Oxford University Press, Oxford. http://dx.doi.org/10.1093/ acprof:oso/9780199544639.003.0001

Wheeler W.M. 1922a. VIII. A synonymic list of the ants of the Ethiopian region. In: Wheeler W.M. Ants of the American Museum Congo expedition. A contribution to the myrmecology of Africa: 711-1004. Bulletin of the American Museum of Natural History 45, American Museum of Natural History, NewYork.

Wheeler W.M. 1922b. IX. A synonymic list of the ants of the Malagasy region. In: Wheeler W.M. Ants of the American Museum Congo expedition. A contribution to the myrmecology of Africa: 1005-1055. Bulletin of the American Museum of Natural History 45, American Museum of Natural History, NewYork.

Yoshimura M. \& Fisher B.L. 2012. A revision of male ants of the Malagasy Amblyoponinae (Hymenoptera: Formicidae) with resurrections of the genera Stigmatomma and Xymmer. PLoS ONE 7 (3): e33325. http://dx.doi.org/10.1371/journal.pone.0033325

Zwickl D.J. 2006. Genetic algorithm approaches for the phylogenetic analysis of large biological sequence datasets under the maximum likelihood criterion. PhD Thesis, The University of Texas, Austin.

Manuscript received: 23 April 2013

Manuscript accepted: 5 July 2013

Published on: 2 August 2013

Topic editor: Koen Martens

Desk editor: Charlotte Thionois

Printed versions of all papers are also deposited in the libraries of the institutes that are members of the EJT consortium: Muséum National d'Histoire Naturelle, Paris, France; National Botanic Garden of Belgium, Meise, Belgium; Royal Museum for Central Africa, Tervuren, Belgium; Natural History Museum, London, United Kingdom; Royal Belgian Institute of Natural Sciences, Brussels, Belgium; Natural History Museum of Denmark, Copenhagen, Denmark. 